\title{
Design for the Future
}

Creating a Champion

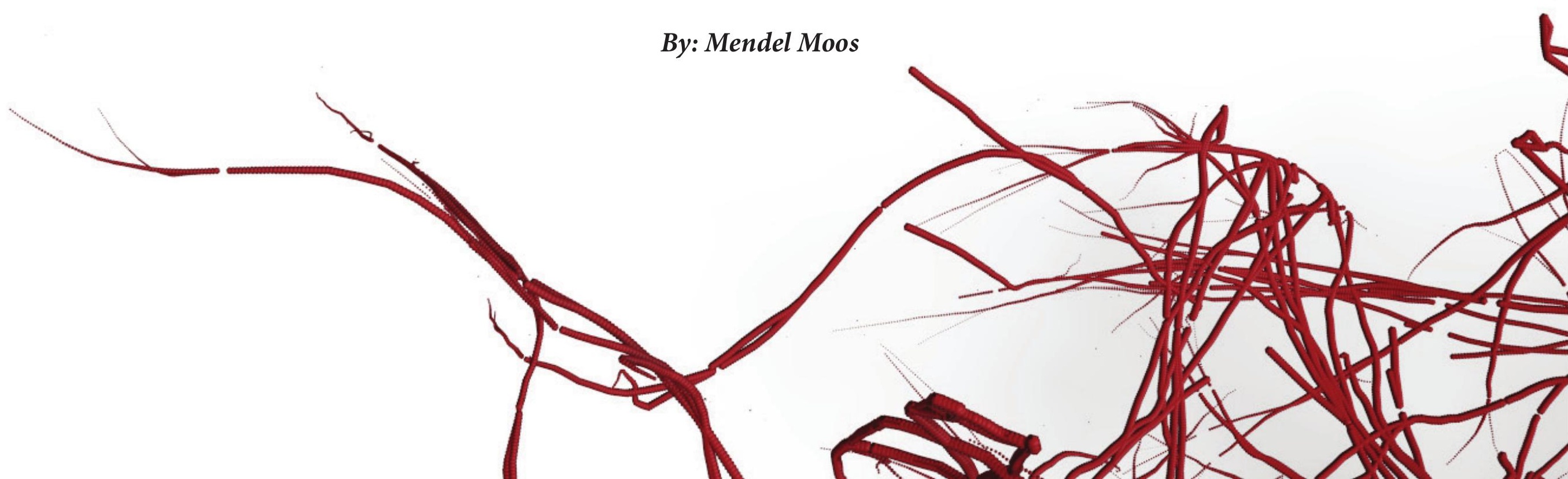


Design for the Future: Creating a Champion

Mendel Moos

A 90-point Portfolio submitted to the Victoria University of Wellington in

partial fulfilment of the requirements for the degree of Master of Design

$$
\text { Innovation }
$$

Victoria University of Wellington

School of Design

2018 


\section{Acknowledgements}

Thank you to my supervisors, Bernard Guy and Simon Fraser for their support and advice throughout my master's journey. You kept me inspired and helped me discover my chosen design path.

To the School of Design staff members Paul Hillier and Keith Davies for your patience and expertise.

To Fenella Richards, for the extra help and critical feedback, you helped me stay on track.

Thanks to the MADE program for the financial assistance.

To Elyse, Tevin and Curtis for their support and contribution to my thesis.

Thanks to Caroldine, Meldin and Madger Moos for their love and financial aid to my path of study. 


\begin{abstract}
The goal of sports protective wear is to maintain safety and reduce the risk of injury, especially in areas of the body most vulnerable during contact. Furthermore, playing an essential role in preserving the longevity and performance quality of athletes. This thesis proposes that the security provided by protective sports performance can also effectively contribute to the confidence and motivation to perform. Thus 'Creating a Champion' is an exploration into what performance and protection could look like in the future, employing contemporary technologies creatively, for a speculative design outcome

Employing 3d scanning, 3d modelling and 3d printing, an effective design approach was developed responding to the design challenge in a practical and appropriate manner. Furthermore, the $3 \mathrm{~d}$ printed concepts served as a protector as well as a sports performance enhancing stimulator. With the application of research through design playing a fundamental role in the design development of the resolved model, the $3 \mathrm{~d}$ printed skin was evaluated and reflected upon.

The thesis was concluded with the $3 \mathrm{~d}$ printed skin performing in new and novel ways. While, providing compelling functionality, and an ergonomic fit to the body the skin was provocative and visually compelling. With minor alterations the composed design may become a product relevant in the future of $3 \mathrm{~d}$ printed sports protective wear. Where protection is a visual and an emotional perception.
\end{abstract}




\section{Table of Contents}

Acknowledgements

Abstract

Introduction

Chapter 1: Background Research/ Literature Review Sports Protection

Sports Performance

Sports Perception

3D Printing

Garment Designing Process

\section{Chapter 2: Methodology}

Research through Design

Design Methods

\section{Chapter 3 Design Process Phase One}

Iterative traditional and digital sketching

Design Approach

\section{Chapter 4: Design Process Phase Two}

Exploring 3d Printing :Part One

Raft and Support

Contouring

Scale skin

Exploring 3d Printing :Part Two

Digital Scales

Growing Support

\section{Chapter 5: Design Process Phase Three}

Anatomy Exploration

Testing the Limits

Battle Scars

\section{Chapter 6: Design Process Phase Four}

$$
\begin{aligned}
& \text { Digital Creation } \\
& \text { Digital Foundation } \\
& \text { Digital Growth } \\
& \text { Tailored for protection } \\
& \text { Final Design Layout } \\
& \text { Virtual to Reality }
\end{aligned}
$$

\section{Chapter 7: Design Process Phase Five}

Resolving the Model

Design Perception 153

Final Design

Physical model

Design Discussion

Figures and Tables 


\section{Introduction}

The goal of sports protection is to maintain safety and reduce the risk of injury to athletes, especially in areas that are vulnerable during contact. Protection also plays an essential role in preserving the longevity and performance of athletes. Protective equipment or wearables have evolved to the degree where they have become customised to the nature of each sports activity, providing players with the reassurance of their safekeeping and well-being. This security can then bring forth certainty that can effectively contribute to the confidence and motivation to perform.

This thesis aims to investigate a unique way of improving sports protection and performance through a speculative design approach and explore ways of equipping athletes with a wearable that can inspire and reinforce championship. It focuses specifically on how $3 \mathrm{~d}$ printed skin can highlight individual identity and memorialise growth to drive athletes to victory through the research and development of textiles and technology. The design objective is to uncover how sports protection can grow from a piece of equipment to a protective skin that signifies this personal identity. It is theorized that the skin has the potential to affect how athletes perceive themselves or how they are perceived by others to improve performance. The aim will be achieved through the application of contemporary technologies such as multi-property $3 \mathrm{~d}$ printing, $3 \mathrm{~d}$ scanning and $3 \mathrm{~d}$ modelling to ensure the functionality and validity of the design.
The thesis will be carried out using research through design as a design methodology, supported by a range of design methods. However, because this thesis focuses on design for the future, a speculative design approach will be applied, encouraging out-of-the-box thinking to produce an innovative and meaningful design. The result will the balance between design investigation and theory-based justification to validate the design outcomes. 



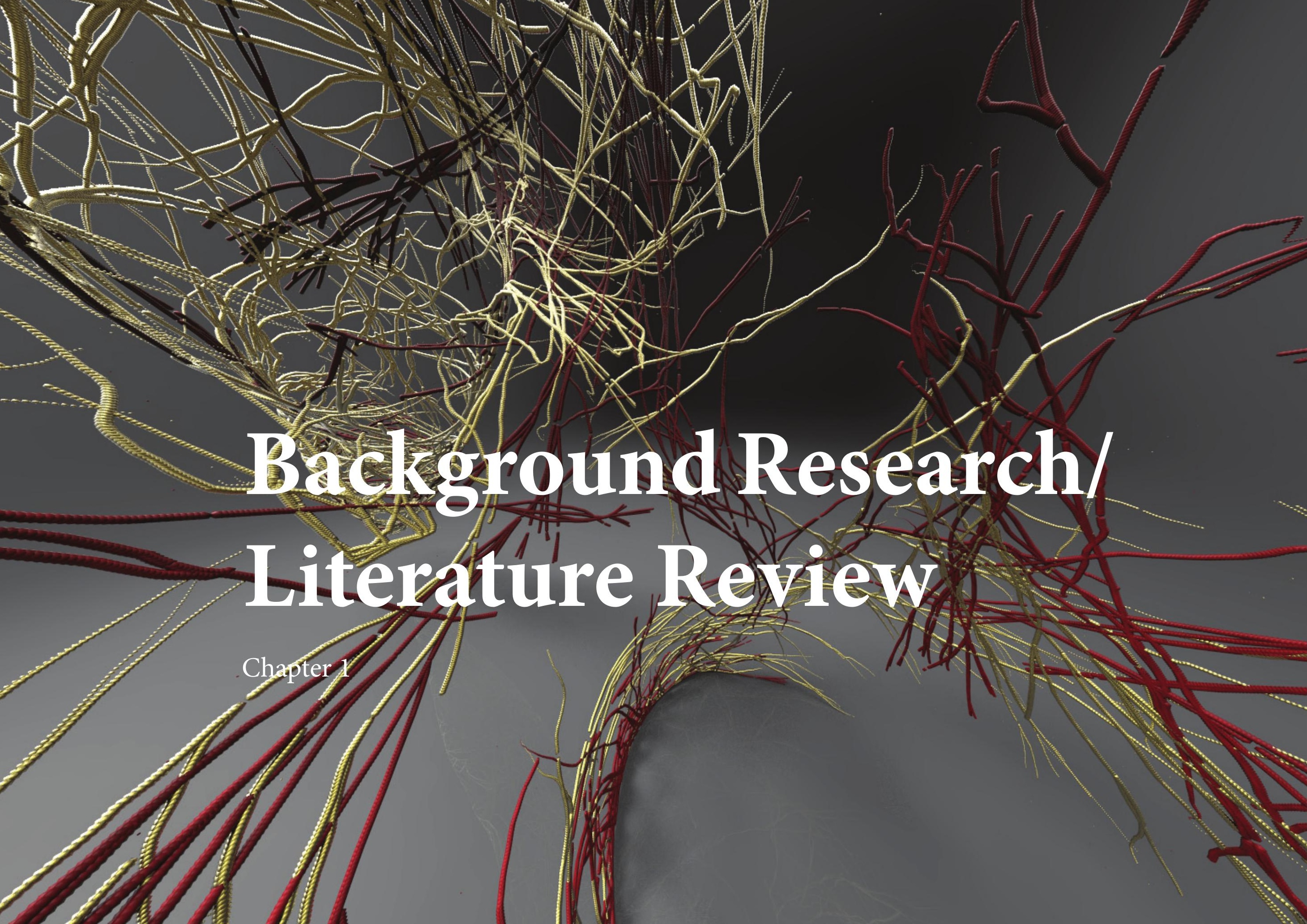




\section{Research Investigation}

The objective of this study is to investigate possible procedures behind the development of a 3D printed performance enhancing wearable. Clothing in high-performance sport has been established to offer protection from injury and is typically tailored to the athlete and branded in complex ways to be visually expressive for the chosen sport. However, the garments mostly remain sewn, with no new development of technologically advanced methods. This investigation will aim to discover ways of recreating and augmenting traditional designing methods through multimedia 3D printing. As well as, analysing current trends and design methods in the sports performance and protection industry to establish a design direction.

The main research topics will revolve around physical performance enhancement, sports protective innovation, emotional stimuli in sports and the creation process of customised wearables. While this project takes a speculative approach to designing a protective performance enhancing suit, the design decisions made are based on proper secondary research findings. This is to ensure authenticity and validity, having the suit perceived to be genuinely functional although no scientific testing has been done. 


\section{Sports protection}

When it comes to most contact sports such as boxing, rugby, American football and hockey, the majority of individuals involved are required to wear some sort of protective sportswear. Due to the high physical impacts endured with in sports such as these, protective sportswear can determine the difference between life and death, especially in sports where high risk of spinal and head injuries are evident.

Industrial and sports clothing is directed by protection requirements and regulations, sports clothing is generally chosen based on performance and comfort. How much protective clothing influence performance is usually determined by the nature of the sport, metabolic rate required, environment, and characteristics of the protective clothing. 


\section{Protective Sports Clothing}

In the Ergonomics and Comfort in Protective and Sport Clothing study, challenges in comfort relative to performance of protective wear was discussed. Stating that the physiological load of protective clothing may impact physical and mental capacity, which is why comfort plays such an important role. On top of this the importance of heat regulating protective wear was also discussed, as the heat stress can gravely impact performance, heat stroke being the third leading cause of death in US for sportsmen.

It's reported that comfort is somewhat associated with performance, shown by a cognitive exam. Comfort was believed to arise from integrated visual thermal, and tactile sensations, psychological status, body clothing interactions and environment. However this may change after a period of wear, for example dampness can cause comfort to decrease. The study evaluates that the best way to improve comfort and ergonomics in protective sportswear lies in the development of new materials and approaches. Materials can tackle problems such as moisture transmitting, vapour transmission rate and increase sweat evaporation. Others can provide better protection with less bulk and weight, such as the ballistic vest protection and so on.
This article brought to light the importance of comfort in sportswear, as well as the significance of using the right materials for the right function. Lack of comfort does not only effect our physical capabilities but also our mental functional, which is something I previously over looked. As sports performance depends on mental strength equally as much as physical strength, a well fitted suit could be essential to optimal performance. If the user feels uncomfortable in the wearable he /she may become distracted and lose focus, causing their ability perform to decrease. Therefore designing a garment which is customised for the user will be essential, additionally design the protective gear to be as minimal as possible while retaining its functionality will also aid in greater performance. 


\section{Common sports injuries}

In order to design an effective protective wearable, knowing which areas to protect and how to address them is a must. Looking at impact sports and the common injuries that are associated with them is valuable, as it allows for a more efficient protective wearable to be designed for the particular sport.

According to Myers Sports Medicine \& Orthopoedic Center the five most common sports injuries are strains/sprains, knee injuries, shin splints, fractures and dislocation. These tend to occur due to poor training practices, improper equipment, flawed techniques or accidental. Moreover these injuries can also occur when a person is not conditioned well enough to perform the sport, such as not warming up beforehand. 


\section{Injuries}

Shin splint is another typical sports injury, this usually involves pain along the front outside of the shin bone(tibia) but can also take place in the foot and ankle (anterior shin splints) or where the bone meets the calve muscles which is on the inner edge of the bone (medial shin splints). This injury is common amongst runners, especially when running on hard surfaces. However these can also be cause if one fails to warm up or stretch, incorrect running techniques, running in shoes that lack in support, or having flat feet can promote this injury. Furthermore fractures is also considered a common sports injury, which is usually caused by a one-time injury to the bone (an acute fracture). However repeated stress on a bone over time can (a stress fracture) can also promote a fracture. Small cracks can complete a break and a stress fracture usually occurs when the legs or feet endure competitive impact from a sports activity, such as running and jumping.

Dislocations occurs when a force pushes the bone out of its joint alignment. This can be cause by contact sports such as rugby or an activity such as excessive stretching or falling. Dislocations can be put back in place, however the connective tissue surrounding the joint could have severe damage. Generally these occur in the fingers, hand and shoulders.

Strains and sprains are the most common type of sports injury, caused when a ligament tears or over stretches. These injuries have a range in severity and are usually found in wrists, ankles or knees. Strains are also known as a pulled muscle, which is when the fibres within a muscle or tendon stretch too far or tear.
Knee injuries are another popular sports injury with over five million people visiting orthopaedic surgeons for knee related injuries and issues every year. Mild knee injuries include iliotibial band syndrome, runner's knee (tenderness or pain near the front of the knee cap), or tendonitis (degeneration or inflammation within a tendon). Furthermore severe knee injuries are usually damage to the cartilage or ligaments. According to Myers Sports Medicine \& Orthopoedic Center four major ligaments that are commonly injured are the posterior cruciate ligament (PCL), the medial collateral ligament (MCL), anterior cruciate ligament (ACL), and the lateral collateral ligament (LCL). 


\section{Protective materials}

With today's advanced technologies, the developments in functional fibres and new innovative structures have contributed to a safer contact sports environment. The majority of protective wear today makes use of ethylene vinyl acetate (EVA) and Polypropylene foam, to act as body armour. However EVA foam does have its structural limitations, although it's soft and provides some cushioning it does frequently bent and crush during movement. Therefore more innovative manufactures today use composites such as D30 and VPD to resolve this complication. These innovative materials provide high impact resistance, high performance and comfort. 
D30 impact additive is a "pioneering ingredient designed to revolutionize back-of-hand TPR impact performance."-D30. D30's material scientists combine polymer blends to achieve specific physical properties such as high shock absorption, abrasion resistance, flexibility and temperature stability etc. In principle D30 is based on non-Newtonian principles where in its raw state the materials molecules flow freely allowing it to be soft and flexibles. However when exposed to physical impact these molecules lock together to dissipate impact energy and reduce the transmitted force. Thus making D30 incorporated protective wear reactive to external forces, which is a very innovative way of making protective wear sensitive without the need of electronic sensors. TPR (total physical response) has been widely used by glove manufactures for construction, automotive and oil and gas industries. However according to D30, increased protection is usually achieved with thicker and stiffer parts, having negative effects on comfort and dexterity. In contrast the "new D30 impact additive combines advanced polymer chemistry that can be blended into existing processing and manufacturing techniques, delivering unmatched impact protection properties to traditional TPR."- D30. After conducted testing done at the D30 Lab, results showed the TPR with D30 impact additive reducing transmitted impact force $34 \%$ greater to traditional a TPR sample of identical thickness. Moreover, D30 states that TPR plastisol enhanced with the D30 impact additive can match the impact protection of standard TPR, while being 34\% thinner. On top of this D30 improves protection, dexterity and comfort as the additive makes TPR 18\% softer. D30 offers a wide variety of design sports protection products adopted by well-known sports brands such Adidas, Under Armour, SCOTT Sports and so on.
POC's visco-elastic polymer dough (VPD) is another innovative body armour material. Similar to D3O the material in its natural state is dough like and highly dampening. However during impact the VPD reacts instantaneously by stiffening and absorbing most of the impact energy. Furthermore due to the material's softness it provides a great deal of comfort and some degree of customization. Though the material is not recommended for extensive use in cold conditions as it is most flexible and pliable when warm. VPD retains the same high impact absorption level and is able to protect users from several impacts during one collision e.g. falling. Due to VPD meeting motorcycle safety standards it's been used in a range of POC's protective gear, from joint to spine protection. 


\section{Protective sports wearables}

To get a holistic view of protective gear within the sports industry, this section will review current innovative wearable designs for high physical impact sports. Focusing on sports such as American football, rugby, hockey etc. as these sports have a large majority of research with in protective wear because of their dangerous nature. Using these products the intent is to assess their problem solving concepts to understand how their technologies can be integrated and with my suit to aid in optimal body protection. 


\section{NFL Helmets}

Due to NFL being one of the most dangerous contact sports as well as its heavy use of protective wear, looking into the most innovative protective gear with in the NFL could provide very valuable insight. After a collaboration between NFL and NFLPA (player association) a coordinated laboratory testing on helmets were carried out by their respective biomechanical experts. With the aim of determining which helmets best reduced head impact severity under conditions simulating potential concussion- causing impacts sustained by NFL players. After this study was carried out the 3 top performing companies and helmet were VICIS Zero 01(2017), Schutt Air XP pro VTD 789902 (2017) and Xenith Epic+ (2017). All three of these helmets make use of very innovative impact absorption techniques which I intend to analyse. 


\section{VICIS Zero 01 (2017)}

The company VICIS is 2017's safest NFL helmet with their Zero 01(2017) model. According the VICIS this model shows significant reduction in impact forces, done through the use of their expertise in medicine and engineering. ZERO1 uses a multiple layered outer shell, with a soft outer shell and an underlying layer of columns designed to mitigate collisions from multiple directions. Providing elite athletes with the best head safety thus far, with the help of their state of the art testing protocols. The engineered multi-layered helmet design reduces impact severity better than competitors based on an algorithm developed for professional football to assess helmet performance. As most helmets only consider linear impact forces in contrast the Zerol considers both linear and rotational forces, therefore improving its impact performance beyond competitors. "The Lode shell (outer soft shell) absorbs impact load by deforming like a car bumper and, then bouncing back"- VICIS. Similar to D30 this helmet also relies on a responsive mechanism, although not to the scientific extent of D30.

To optimize fit, a data -driven approach was used through the use of helmet pressure maps. Due to the neurosurgeons at VICIS, there was a deep understanding of player head shape and anatomy. Using this idea could be very interesting in deterring the pressure needed at each location of protective padding. Which may not only benefit protection but also blood flow and therefore increased oxygen intake for greater performance. VICIS used their AXIS system which uses head length and breadth measurements to allow for the perfect fit with pressure eliminating the need for shims and air bladders. On top of this VICIS ZERO1 also provides greater field of view compared to its competitors with almost 5 degrees more than the next best traditional helmet, improving awareness and thus the safety and performance of elite players. After analyses of several impact padding or structures I have come to the realisation as to how important this impact absorption is. Products and companies who tend to focus on ways of improving ways of dissipating the exerted energies seem to perform the best within the industry. 


\section{Sports performance}

Today Enhancing sports performance at elite and participatory levels, requires the combined efforts of athletes, coaches and even scientists along with other professionals. With a large majority of sports and exercise science focused research and examination of how science and technology can promote enhanced sports performance. Currently research into human performance focussed on analysing how the human body moves, from the internal operations such as the muscles and skeletal system to how this translates into external movement execution. From these techniques, principles are applied to understand how athletes function and learn to stimulate improvement. On a regular basis coaches and athletes try to balance intensity and frequency to obtain the greatest enhancement in performance. This is where technology and science have improved sports, with accurate monitoring sensors, coaches and athletes are able to correctly determine intensity (stress), frequency (overtraining) and therefore optimise sports performance. 


\section{Athletic Training}

The trend of using real-time wearable devices is growing exponentially with in the sports performance industry. This is due to the immense benefits they provide in terms of assessing physical activity, as well as being driven by their increased availability, lower cost, and advancements of personal computing devices such as smart phones and digital watches. Allowing athletes, sports teams and physicians to monitor functional movements, workloads and biometric markers to improve performance and reduce injury. The most commonly used movement sensors include global positioning sensors (GPS), pedometers and accelerometers/gyroscopes devices. Physiological sensors include sleep monitors, heart rate monitors, temperature sensors and integrated sensors. This section of my study will review current utilization of wearable sensors and their potential for future integration sports performance.

In sports performance these devices have become quite valuable, allowing performance variables to be monitored. Making the identification of biochemical fatigue a lot easier, permitting athletes and coaches to avoid injury during training. Furthermore, these monitoring wearables also promote the development of optimal training regiments for each individual maximising athletic performance. The constructive real time information can also be used to detect position specific patterns in movement, design customised training programs, optimise performance and detect possible injury causes such as concussion and fatigue. 
The article 'Textile-based wearable sensors for assisting sports performance' discussed a range of wearable sensors which can be used to asses physiological signals and body kinematics during exercise. Addressing ways to integrate such sensors within garments allowing users to monitor progress throughout their exercise programs. Using textile based sensors which are compatible with textile manufacturing processes, in turn making the fabric itself a sensor whilst maintaining the functional properties of the garment. Evaluating the $\mathrm{pH}$ sensor which analyses sweat in real-time, a novel approach to chemical sensing on fabric. As well as assessing the piezo resistive sensors and its response to body movement. The paper looks into fabric circuitry and sensor integration issues. The importance of rehydration for athletic performance mentioned and ways of indicating sweat loss to ensure hydration. This is done through using a $\mathrm{pH}$ sensitive indicator which has a colorimetric response along with LEDs to analyse sweat directly in real-time and provide feedback. Looking into physiological monitoring in particularly breathing patterns to calculate how hard the body is functioning. The wearable unobtrusive sensor permits the measurement of breathing patterns indicating performance and recovery to exercise. The benefits of improving breathing techniques is great when it comes to health and wellbeing, especially with those affected by stress.
Body kinematics was discussed in terms of postural alignment for motor skills training as athletic movement relies on dynamic movements. Using fabric strain or pressure sensors, body kinematics can be assessed through monitoring joint movement and foot plantar pressures which could create improved technique through better body awareness. Moving onto fabric circuitry as a control system to power sensors, capture data and communication of this information to the user productively. The paper shows an advantage of using conductive piezo-resistive materials is that they can be connected directly using conductive thread. Using the Lilypad system a connection can be dispersed within a modular structure throughout the garment by means of stitching conductive thread in place. However an issue with using stainless steel conductive threads is the tendency of fibres fraying which can cause short circuits, making it important to insulate connections. 


\section{Primers}

During a meeting with the Silver Ferns strength and conditioning coaches Stephen Hotter and Adam Allen, when asked how they prevent injury during a netball they mentioned primers. Primers is essentially a warm up which involves compound movements e.g. squats or deadlifts. Through doing primer movements prior to their netball match players are able to lubricate joints, activities musculature and sharpen movement patterns before participating in an activity with near maximal effort. Although athletes are able to lower risk of injury by doing this they are also able to improve performance. By activating more muscles and greater joint movement this will result in enhanced muscle and joint function, providing smoother, precise and more powerful movements. To address this through my wearable design could be very beneficial, providing athletes with an option of using primer workouts without having to go to the gym and use heavy weights which again could lower risk of injury. A garment that is convenient (e.g. being able to use it closer to the actual event), effective and efficient in preparing the athlete to perform at a high level.
EXOS

EXOS is a human performance company, helping people reach higher and achieve more. Founded in 1999 with the belief that human performance is for all of us, with the focus on healthcare, corporate wellness and sports performance. In terms of performance EXOS focuses on several steps. Learning and understanding every individual's aspirations, designing solutions based on the needs of their clients then measuring and refining systems to produce optimal performance. EXOS is well-known for working with elite NFL players and Olympic athletes providing sports performance training, nutrition, rehabilitation and sports science services. Believing that to optimize performance both physical and mental weakness needs to be addressed. The key to EXOS success is ensuring aspect of performance is looked into, through research and personal consultation, and providing athletes with a customized program to increase the chance performance enhancement. 


\section{Biosensing Wearables}

According to Rock Health analysis of this market shows potential significant growth, with an estimated market size ranging from $\$ 5.8 \mathrm{~B}$ to $\$ 50 \mathrm{~B}$. Due to the large smartphone adoption it has allowed wearable device makers to rely on smart phones for display computing, internet connectivity and in turn reducing the price of wearables. However despite great technological advancement in the wearable tech industry, it fails to engage users effectively. Analysis showing the rate of engagement decline drastically during the first 6 months of ownership. Rock Health discussed the top three wearable trackers Fitbit, Jawbone Up and Nike Fuelband which represent $97 \%$ yet also fails to send clear marketing messages. In order to combat low engagement rates and scaled beyond early adopters companies need to satisfy three key factors appropriately - functionality, reliability and convenience innovatively. Functionality defines a biosensing wearable's potential utility to an end user, without this usefulness to the consumers become highly limited. Reliability influences addressable segments due to unique constraints of operating in a healthcare environment as customers demand valid data to inform clinical decisions. On top of this convenience determines engagement of biosensing wearables, without convenience user engagement fails. These three keys aid largely in product performance within the healthcare market. As this market becomes more competitive companies will continue to innovate and eventually create products that meet the demands of traditional healthcare markets 


\section{Cryo, Thermo, Massage Therapy}

Muscle damage is a big limiting factor for muscle performance during days post exercise, therefore different methods of decreasing delayedonset muscle damage have been discusses including massage, stretching, compression, anti-inflammatory drugs, antioxidants exercise and cold water immersion (Journal of Sports Sciences.2010). Thermotherapy and Cryotherapy are treatments which have been well documented throughout the years, due their benefits in terms of muscle recovery. Incorporating these hot and cold treatments into the smart wear will help prevent injuries and improve the muscle recovery process.

Cryotherapy is defined as the therapeutic application of any substance to the body that removes heat from the body, resulting in reduced tissue temperature (Pain Physician.2004). For this reason it is known to decrease blood flow within soft tissue due to vasoconstriction. Furthermore reducing tissue metabolism, oxygen utilization, and inflammation and muscle spasm. Studies found that the use of intermittent compression and intermittent ice water treatment (47\% swelling reduction) proved to be more effective than continuous ice water treatment (33\% swelling reduction).However complications can arise when application comes in contact with superficial nerves which could lead to major injuries such as lateral femoral cutaneous nerve injury especially when used with compression. Depending on the cryotherapy application method the treatment may have different physiological effects for example analysis found that an ice massage cools the muscle faster than an ice pack (Pain Physician.2004).
In contrast thermotherapy that adds heat to the body resulting in increased tissue temperature, therefore increasing blood flow due to vasodilation, metabolism and connective tissue extensibility. Increasing blood flow facilitates tissue healing by supplying protein, nutrients oxygen at the injury site. Furthermore increasing local tissue metabolism by $10 \%$ to $15 \%$ improving our healing process by increasing both catabolic and anabolic reactions needed to degrade and remove metabolic by-products of tissue damage and provides the milieu for tissue repair(Pain Physician.2004). Heat therapy also effects the brain causing increased activation of the thalamus and posterior insula, effecting the brain directly can decrease the sense of pain.

Journal of Science and Medicine in Sport 2009 shows that post-exercise cold water immersion to be beneficial for muscle recovery Analysis illustrates lower muscle soreness, reduced inflammatory response and consequent secondary muscle damage. "Cryotherapy has been shown to reduce cell necrosis, oedema and neutrophil migration, as well as slow cell metabolism and nerve conduction velocity, which in turn reduces secondary muscle damage". The study states that benefits may have been facilitated by warm water endorsing vasodilation, which improved immune function by increasing antioxidant and antibody supply, while cold water promoted vasoconstriction, reducing swelling and myocellular necrosis. Another study shows that players who undertook cold water immersion directly after the match reported lower perceived muscle soreness. 
One cause of fatigue is the restriction of blood flow to active muscles therefore providing insufficient oxygen and inadequate removal of metabolic waste products. A study by Medscimonit shows that blood and lymph flow to be a common physiological effect derived from muscle therapy. Massage therapy helps remove metabolic waste by-products such as lactic acid which in turn aided in the recovery of muscle fatigue. Studies found that massage therapy was successful in reducing the magnitude of DOMS (delayed-onset muscle soreness), swelling and plasma CK activity. Additionally if a massage is performed post-exercise but before DOMs develops can reduce soreness by $30 \%$, no matter how the massage is performed (Journal of Athletic training). Using massage therapy in combination with thermotherapy and cryotherapy with possibly a soft robotics module we can drastically speed up the recovery process of athletes and therefore improve their athletic performance. 


\section{Compression wear effectiveness}

In fitness and sports, compression garments have become very popular among athletes ranging from recreational to professional with the intention of improving performance, injury prevention and injury rehabilitation. With sports teams such as the All Blacks adopting their new jerseys with a supposedly ground-breaking chassis system and new fabric innovation designed by Deborah Yeomans, director for Adidas futures. "In looking at the kinetic chain, which is the flow of energy through the body, we found an area we could improve to give the All Blacks a competitive advantage..." Yeomans says. However research shows there to be minimal physical performance benefits ability with the use of compression garments but rather its biggest beneficial effects to be for post -exercise recovery purposes. A study done by MacRae, B.A., Cotter, J.D. \& Laing, R.M for the sports medicine (2011) suggests that all though minimal, compression garments can attenuate muscle oscillation, improve joint awareness, alter sub-maximal oxygen usage during exercise, alter local blood flow and protein or metabolite clearance, mitigate swelling and reduce perceived muscle soreness during post-exercise recovery. Compression wear was initially introduced with a focus on venous thrombosis, with an aim of improving blood flow around the body. Which is shown through a study done with lightweight gradient compression stockings, and findings showed that using a gradient compression hosiery is very effective in improving symptoms of discomfort, swelling, fatigue, aching as well as leg tightness (Robert A. Weiss MB, David Duffy MB). 


\section{Perspiration}

Analysing the physiological effects perspiration has on athletes and the importance of water in our bodies, additionally maintain the perfect body temperature for limited perspiration. The body uses water to maintain blood volume, to regulate body temperature and also plays a role in muscle contractions. Consumption of liquids aids in replenishing/restores fluids lost and maintains proper muscle function, therefore supporting physical performance and reducing the risk of heat stress.

Research findings show that athletic performance shows that sweat loss equivalent to $2 \%$ of body weight caused a noticeable decrease in physical and mental performance. Sweat loss exceeding $5 \%$ of body weight can decrease work capacity by 30\% (Jeukendrup, Asker E., and Michael Gleeson.2010). Endurance showed greater negative effects in hot environments than in cool settings. Which means thermoregulation does play a role in reducing exercise performance in correlation with body-water deficit, even during low-intensity exercises. Thus reducing exercise endurance from 121 minutes to 55 minutes as well as a decline in core temperature athletes were able to tolerate. This occurs due to the physiological dehydration effects such as reduction in blood volume, decreased skin blood flow, decreased sweat rate and heat dissipation as well as an increased core temperature and rate of muscle glycogen use (Jeukendrup, Asker E., and Michael Gleeson.2010). During exercise the combination of environmental heat stress and dehydration can limit cardiac output and blood delivery to active muscle groups impairing athletic performance. Body temperature rise at a faster rate due to the inability to lose heat when dehydrated, therefore critical temperatures are reach quicker during exercises. Furthermore putting emphasis on assisting body's temperature regulation process in attempt to limit perspiration.

Although sweating is effective in regulating our body temperature at times the volume of sweat produced can become too great and leaving clothing soaked. Today performance wear such as SKINS treats this through the wicking of perspiration by drawing sweat away from the skin for it to be evaporated on the surface. However through this process of wicking Mike Smoltz, founder of TR Gear says that by drawing sweat away from your skin your body won't cool properly, instead it will make you sweat even more, leading to dehydration and compromised muscle performance. Using innovative ways of dealing with sweat whilst not impairing but assisting the body's temperature regulation process will be beneficial for enhancing athletic performance. This could be done through using fishnetlike textiles where sweat can be controlled with minimal absorption while still allowing sensors to collect data through perspiration. 


\section{Sports Perception}

Physical abilities, professional skills and even personality traits are considered important in athletic success. However the significance of emotion stability is also considered a vital part in achieving optimal sports performance today. Shiraz University of Medical Sciences conducted a study to determine the impact of mental happiness on athletic success. Looking at mental happiness in terms of emotional response, satisfaction and general judgement about life. Defining happiness as "the predominance of frequency of the occurrence of positive emotional experiences over negative ones.. Stating that if positive emotion outweighs negative ones, individuals enjoys more mental health and functions appropriately. However, in the opposite case, mental health is reduced causing the individuals functioning to be disrupted. Therefore the study was conducted to analyse the relationship between positive emotion and sports competition outcome. After in-depth study with a large demographic range of participants the study showed some interesting results. Results showed that mental happiness can ultimately predict $42 \%$ of athletic success. The increase of mental happiness was found to greatly determine the success in both individual and team sports, moreover the results were not affected by the type of sports. The study also found a positive correlation between emotional intelligence and athletic success. Indicating personality features, social intelligence, and mental happiness can substantially affect success, and that mental happiness can best impact the individuals ability to achieve their goals (educational, competitive, athletic, etc.). Meaning that the mental state of an athlete before and during a sports activity can impact their decision making which will ultimately affect their performance. 


\section{Colour Perception}

Colour has been known to influence our emotions, decision making, judgement and perception. A considerable amount of scientific research has been done around how colour may affect our brain processing and the functioning of humans. Going back to German poet and polymath Johann Wolfganag von Goethe and his work "Theory of Colors" Goethe $(1810 / 1967)$ presented intuition-based speculative theories on the link between colour perception and psychological/emotional functioning. Proposing that colour perception produces physiological reactions in the body which can provoke people's emotions, cognitive focus and motor behaviour. Goldsteins ideas were formulated by subsequent researchers analysing his ideas on the link between wavelength and arousal. Findings showed that that longer wavelength colours such as red and orange activated the experience of arousal and warmth, whilst shorter wavelength colours such as green and blue experienced relaxation and coolness. These colour induced emotions were thought to impact performance on the achievement of task difficulty. Where longer wavelength colours relative to shorter wavelength colours inducing states that impair performance on complex tasks but improve performance on simple tasks.

In 1978 Alexander Schauss and his research assistant John Ott carried out extensive research into the effect of colour on emotions. Schauss and Ott observed that colour encouraged physical reactions to produce in the body manifesting in observable behaviour. They concluded that a particular shade of pink which he labelled P-618 triggered some insightful effects. Proposing that it had an endocrine-based weakening effect on muscle function and even influenced the cardiovascular system, on the other hand blue had an endocrine-based strengthening effect on the mus- cle functioning. Schauss stated that by simply staring at an $18 x 24$ inch card printed with this particular pink colour, especially after exercising, "a marked effect on lowering the heart rate, pulse and respiration as compared to other colors." In 1979, Schauss convinced the directors of a Naval correctional institute in Seattle, Washington to paint a few prison cells in this pink and analyse its effects on the behaviour of prisoners. These cells were monitored and according to the Navy's report, "Since the initiation of this procedure on 1 March 1979, there have been no incidents of erratic or hostile behaviour during the initial phase of confinement" Adding that only fifteen minutes of exposure were required to reduce potential aggressive or violent behaviour. Later on Schauss named the colour after the Naval correctional institute directors Baker and Miller, calling it BakerMiller Pink with the RGB code: R: 255, G: 145, B: 175. 


\section{Colour in sports}

Hill and Barton (2005) used data from combat sports in the 2004 Olympics to test their proposal that red functions as a dominance cue in human competitions and enhances performance as a result. Findings showed competitors who wore red sportswear relative to blue were more likely to win the competition, which was most evident in male competitors. This was also applied to elite English soccer leagues and multiplayer first-person shooter video games, which evidently produced a similar result. However red was not found to boost performance in all countries, perhaps due to culture-specific associations. Furthermore, it was found that the effect of wearing red may enhance one's dominance, aggressiveness, testosterone, and pre-performance strength, which in turn facilitates competitive outcomes. On top of this, studies found that the influence of wearing red and or viewing red on an opponent may also provoke an influence. As demonstrated in several studies opponents in red are perceived to be more dominant, intimidating etc. demonstrating a general association with red and aggression.
In a study done by Mark G. Frank and Thomas Gilovich at Cornell University, a survey was carried out to analyse the impact black uniforms have on professional sports. Due to the association of black with evil and death in most cultures, Frank and Gilovich were interested in finding out whether this may impact sports performance and behaviour. After the study was carried out with professional football and ice hockey teams who wore black uniforms, results showed that these teams were more aggressive than those with non-black uniforms. Wearing a black uniforms can increase a person's tendency to engage in aggressive behaviour. An analysis of penalty records indicated that teams with black uniforms in both sports ranked near the top of their leagues in penalties. To ensure accuracy, when teams switched from non-black to black uniforms, an immediate increase in penalties were presented. This was believed to be associated with social and self-perception processes.

Baker Miller Pink hoodie

This phenomenon still inspirers use in today such as design such as the Baker Miller Pink relaxation hoodie by Vollebak. Engineered for relaxation and recover through use of Baker Miller Pink and sound to activate parts in the nervous system that aids in rest and recovery. Slowing your pulse, breathing and brainwaves, helping users conserve energy preperformance, maintain focus during events and recover faster afterwards. 


\section{Inside the mind of champion athletes}

In a Ted Talk by Martin Hagger a professor of psychology at Curtin University, sports psychology was discussed. With and expertise in social, health, sport and exercise psychology, Hagger explains how the mind of champion athletes at highest levels. Elaborating on how his involvement in multiple research projects, focused on motivation and behaviour change unveiled what triggers optimal sports performance. Explaining that physical abilities, skill and conditioning are often not enough to achieve and perform in high stakes situations such as the Olympic games. However, arguing that using and creating strategies to preparation is the best way to ensure athletes are in the best condition physically as well as psychologically to perform at peak levels. Hagger explains sport psychology to be the science and practice of mental preparation for sport. Involving the identification of techniques and strategies that athletes can take and use to perform at their most optimum (Hagger,M.2013). On top of this helping athletes deal with setbacks and recovering from devastating defeats, such as Rory Mcilroy who lost the final 2011 Masters Tournament after losing his four-shot lead, but eight weeks later winning the US Open Golf. Hagger explains the factors that contribute to high performing performance being motivation, confidence, performance knowledge, routines and anxiety management. Hagger states the importance motivation but how motivated an athlete is, can defined by the goals set. Determining how much will and drive an athlete have to perform at their best. However, motivation on its own is not enough, self-confidence is vital to peek performances. One of the ways this can be done is through imagery and or mental rehearsal, Hagger elaborates. Visualising the race/match, imagine the sensations as well as any contingencies that may arise. Anxiety management is also mentioned,
Hagger explains how relaxation Techniques such as self-talk, meditation, breathing, music etc. For example, Michael Phelps used music before his race to help him enter the right frame of mind. As this routine helped him stay relaxed and allows him to manage his anxiety (Hagger,2013). Hagger concludes by stating from a sports psychology perspective an athlete needs to be motivated, confident in their abilities, training, preparation. Also manage pressure well, and uses well-drilled techniques to prepare such as imagery, self-talk and relaxation. 


\section{D Printing}

3D printing also known as form of an additive manufacturing process, allowing three dimensional physical objects to be created from a digital file. An additive process usually involves a process of laying down several layers of a range of materials until the physical object is completed. Although in some cases $3 \mathrm{D}$ printing may be more expensive than other traditional techniques such as injection moulding, it can provide greater complexity, flexibility and functional parts which are impossible to create with conventional techniques to be created.

3D printers today are used in multiple different fields, however the 3D printing of wearable textiles are still quite a niche market. 3D printed textiles have been experimented with, for example studies around biomimicry experimentation through the use of 3D printing have been done effectively. However not a lot of this have been designed for everyday use but more for show or demonstration. Therefore this project focuses on this currently niche market, and ways of introducing the $3 \mathrm{~d}$ printing into sports activities. 


\section{$3 \mathrm{D}$ printing on textiles}

A study called 'combining 3D printed forms with textile structures mechanical and geometrical properties of multi-material systems' done by Niederrhein University of Applied Sciences focused on the integration of 3D printing onto traditional textile structures or fabrics. Looking into the application of 3D printed forms in garments through merging them with textile fabrics ensuring tensile strength. Taking different approaches of combining 3D printed polymers with different textile materials and fabrics and assessing the limits of this technique. Examining the possibilities to print 3D forms directly onto textile fabrics which differs from previous experiments where the focus was on creating textile-based structures from different polymeric materials through FDM printing. As 3D printed additions to garments should be soft and not rigid, PLA soft was used as the filament material over the commonly used ABS.

Results derived from the first test series revealed that 3D printed forms can be easily stripped from fabrics. Although the polyester net showed promise as the molten PLA flows around the single threads and encloses them, allowing both parts to connect. Adjusting pressure and temperature to a higher magnitude with the aim to improve adhesion proved pointless as the printed patterns were still easily stripped from the textile fabric. Therefore focus were relocated to "open" textile structures such as nets which proved successful in the first test. Examining these connections between the printed layers and textile fabrics through microscopic pictures of cross sections a problem was discovered. Which was an undesired lateral shift of the layers with respect to their neighbours. Therefore adjustments to printing parameters were made to enhance layer- layer adhesion as well as line-line adhesion. In conclusion to combine tensile strength with of textile fabrics with 3D printed forms, several textile nets was found to be sufficient with the connection between both materials. 


\section{D printing Biomimicry}

Biomimetic or biomimicry is the approach to design innovation that seeks sustainable solutions to problems through imitation of nature's systems, structures, function, and elements. Humans have always looked at nature as a way to inspire problem solving, for example the first airplane an so on. I believe biomimicry could play a large role in my project, in terms of looking at enhanced performance, protection and even emotion stability. These there can all undoubtedly be linked back to our human survival instincts, which nature has played a large part in shaping, since the beginning of mankind. Therefore I believe mimicking natures organic structures can aid in greater protection and performance, and investigating its forms and colour as to how they are perceived by us.

With 3D printing technologies we are able to construct simplified physical models of complex biological systems, allowing us to investigate their mechanical properties. For example a study done by Clemson University (USA) and McGill University(Canada) investigated the bio-inspired 3D printing focusing on the dermal armours of fish and how their mechanics. Finding that "Mimicking the overlapping arrangement of elasmoid like scales revealed that scale rotation, bending, and frictional sliding facilitates varying amounts of resistance to penetration (protection) and body mobility (flexibility), while simple geometries that create a topological interlocking effect between adjacent scales further increases their penetration resistance". Also analysis of shark skin showed that adding denticlelike microstructure on the surface of a flexible layer can decrease drag and increase swimming speed as demonstrated by Speedo's LZR Racer swimsuit design which was later banned from the Olympics. 


\section{Garment Design Process}

With the increased advancement in virtual reality application, the garment industry has developed new garment designing technologies and techniques. Using garment CAD technology which is the use of computer technology to aid in design of garment products. In comparison to mechanical product, garment CAD have resolved special issues. Such as modelling soft material with low bending stiffness, rather than rigid inflexible objects. Garment components can be assembled through following specific pattern making rules, on top of this although the garment is designed and constructed from $2 \mathrm{D}$ patterns, the quality of fit can be evaluated on 3D human models. Typical commercial 2D garment CAD software's include Toray-Acs in Japan, Gerber in United States, Investronica in Spain and Lectra in France. Although the high reliance pattern masters experience in 2D systems, 3D garment CAD methods with the inclusion of human body measurement technologies are often recommended as an alternative to non-expert users. With main techniques being 3D human body measurement and modelling, 3D garment design on digital human models, 3D draping simulation and $2 \mathrm{D}$ pattern generation from a $3 \mathrm{D}$ space. Companies well known for this 3D garment systems are AssystBullmer in Germany and Dessingsim in Japan.
In a survey on $\mathrm{CAD}$ methods in $3 \mathrm{D}$ garment design the process and approach of designing a 3D garment was discussed. Proposing current methods as to how to construct a 3D garment through the use of current 3D garment technologies and techniques. Moreover with the advancements in $3 \mathrm{D}$ scanning technologies today it provides designs with an easy way of capturing geometric information of their designated human model. On top of acquiring the geometric parameters of the human model it is necessary to use semantic features to facilitate the garment design on the $3 \mathrm{D}$ model. Two sets of sizing parameters are required, including height, specified by parallel cutting planes and girths, measured by the cutting plane intersecting the human body. Through the use of cutting planes we can specify and classify vertices, edges and faces in the body mesh model. By doing this we're also segmenting the human body allowing for parameterization and providing accurate model measurements. Special characteristics of the human body are also considered in establishing locations for cutting planes. Such as using the crotch point which is usually $5 / 8$ total height from the top. 


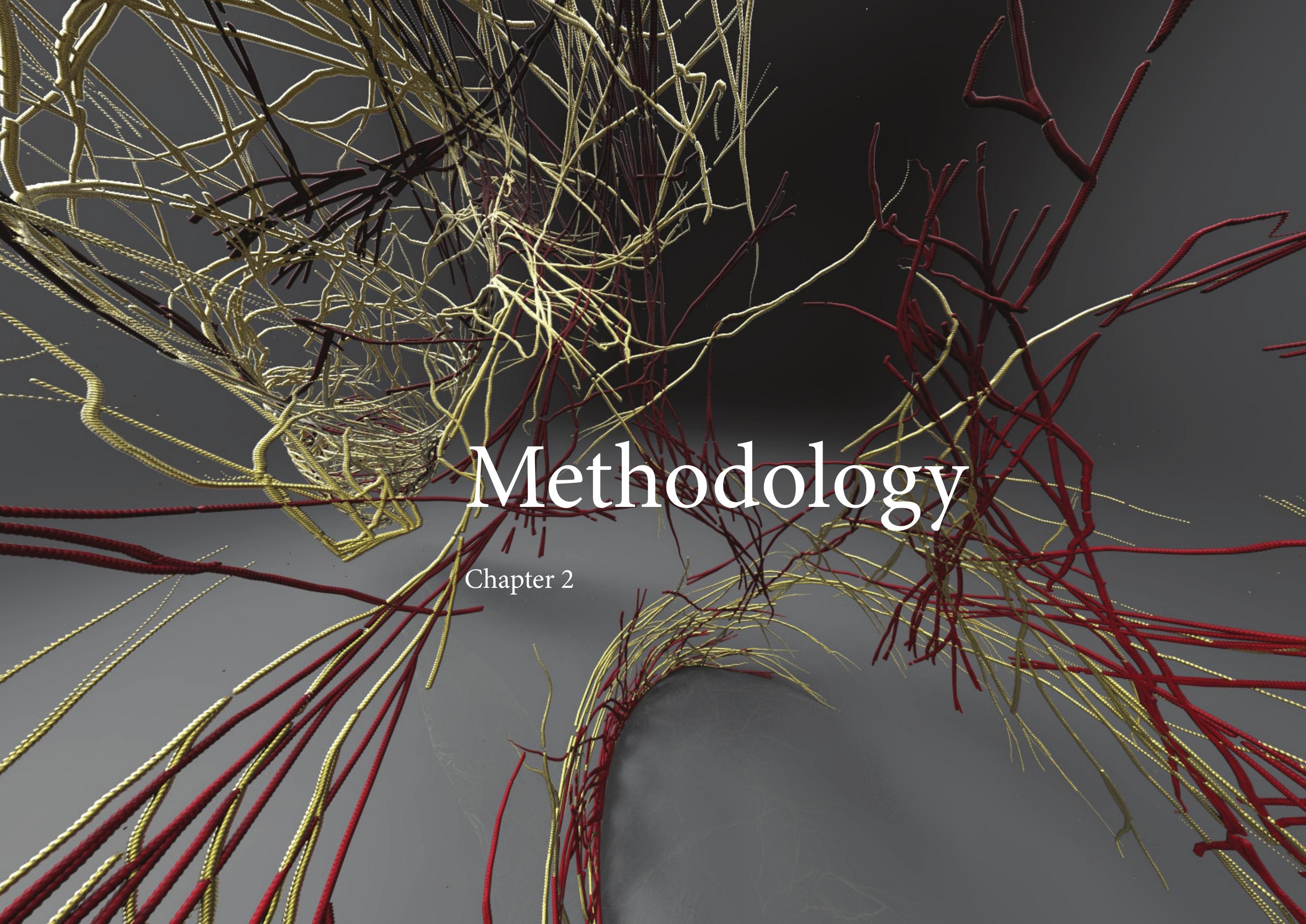


Thischapter discusses the primary methods applied throughout the research design process. The research conducted were based predominantly on an engaging iterative experimentation design processes. Through constant prototyping and design reflection of physical objects the objective was to demonstrate an innovative design approach that enabled the production of successful physical designs. However to ensure an effective design process the appropriate design tools and methodology was required. Therefore, the primary methodology used was Research Through Design. Utilizing this methodology preserved creativity as well as assuring validity through design research and reflection. 


\section{Research Through Design}

Using research through design this project investigates the potential of a 3D printed protective performance enhancing wearable. Applying methods to design phases to distinguish the most appropriate way of integrating the researched elements and in theory produce an effective speculative design. Focusing on improving the protection and performance of athletes physiologically through aesthetic and function. Using an iterative design research process the aim is to create a functioning design model, following the four phases by Daniel Edelson.

1. The development of a theory.

2. The derivation of principles for design from the theory.

3. The translation of principles into concrete designs.

4. The assessment of design to test whether they work as anticipated.

Traditionally, design in educational research has served as a way of implementing theories for testing (Edelson,D). The design research methodology, research through design, treats design as a strategy for developing and refining theories. The research through design paradigm produced by Edelson encourages lessons to be learned through design. Encouraging the designer to become in engaged in problem analysis, design solutions, and design processes. Through this research model, Edelson argues the validity of using design research methods as a form of educational research. Stating that (a) design offers opportunities to learn unique lessons, (b) design research yields practical lessons that can be directly applied and (c) engages researchers in the direct improvement of educational practice.
Due to the complexity of the process of design, open-endedness and dependence on creativity, makes it difficult to explain. Design is a sequence of decisions balancing goals and constraints (Edelson, D. 2002). Throughout any design process, three decisions are made determining the design outcome. These involve, (a) how the design process will proceed, (b) what opportunities the design will address, and (c) what form the resulting design will take. As stated by Edelson the design procedure identifies the processes and people involved in the development of the design. This may involve establishing specific design processes which respond to a particular design challenge. The problem analysis, involves the goals, need, or opportunity that a design is intended to address together with the challenges, constraints and opportunities presented by the design context constraints (Edelson, D. 2002). Generally, a design process initiates with an alleged problem or opportunity, and an idea for how to respond to it. However, the initial idea is normally elaborated upon through a combination of reflective or analytical processes. Furthermore, the finalised design solution embodies the resulting design. Reflecting the results of the designers' efforts to address the challenges, satisfy constraints, utilize opportunities and balance compensations that were recognized in the problem analysis (Edelson, D. 2002). The employment of this methodology becomes appropriate as this thesis focus on investigation of how applicable3d printing may be in regards to the sports protective industry. 


\section{Design Methods}

\section{2,3d Traditional and digital sketching}

Sketching throughout this thesis played a key role in the initial conceptual designing process. Allowing for concepts to be generated and refined frequently through visual thinking. Design sketching differ from 'drawing an object' as they are not something which is pre-existing. Instead it allows the designer to become involved in a process that attempts to give external definition to an imagined, or half imagined, suggestion for design form and function (M.Tovey, S.Porter, R.Newman.2003). Sketches will provide a way of handling design ideas, permitting greater understanding processes such as design thinking, problem solving and communication. Through utilizing iterative sketching the ability to envision concepts without physical modelling is possible. Promoting quicker problem solving and refinement of a design theory based on design reflection.

\section{Photography}

Photography is a very effective way for both documentation as well as investigating events that has occurred. Therefore, photography was used throughout the design process for communicating the design process. Through photography designs were analysed, critiqued and evaluated consistently. Photography methods such as chronophotography wer utilized to investigate the human body within the specific design context. Providing a holistic understanding of the design challenge as well as determining effective ways to respond to it. This method played a large role in evaluating how the physical models behave and look in the given context, encouraging critical design thinking.

\section{D Scanning}

Through $3 \mathrm{~d}$ scanning, analysis of a real-world object can take place providing a collection of data on its shape and appearance. The gathered data can then be applied to construct a digital three-dimensional model. $3 \mathrm{~d}$ scanning grants an opportunity to digitally design $3 \mathrm{~d}$ models from a realistic human anatomy, as well as catering for customization in regards to ergonomic fit. Furthermore, providing a foundation for the $3 \mathrm{~d}$ modelling process, and enable designs to grow from real world geometry within a digital work space.

\section{D Modelling}

Computer- aided design (CAD) is the use of computer systems to aid in creation, modification, analysis or optimization of a design (Wikipedia.2018). CAD will be practised in order to produce detailed $3 \mathrm{~d}$ models throughout the design process. This will predominantly include designing the initial design concept iterations for $3 \mathrm{~d}$ printing testing. However, 3D computer graphics, 3D modelling will be utilised to create the final outcome as it allows for greater design dynamic in regards to creative artistry. $3 \mathrm{~d}$ modelling, provides the ability to sculpt models from geometry of the body preventing static design as well as accommodating in depth evaluation of aesthetic and function. 


\section{D Printing}

Through $3 \mathrm{~d}$ printing, designs will to be translated from digital to physical. Allowing for extended design reflection to be employed, as the ability to analyse physical models through touch can be applied. Thus, physical models can bring forth problems which may not become visible during the digital designing process. Permitting analytical critiques and evaluation of physical models based on the design purpose and context.

\section{Rendering}

Rendering provides the ability to envision concepts more in depth and life-like manner, without the need to produce physical models. Through this method, textures and colours can be applied to the $3 \mathrm{~d}$ modelled surface. As this thesis practices a speculative design approach, the value of using rendering is increased. As certain design qualities may not be able to be created with the given design tools, renders can be used as a substitute. Moreover, creating renders will not only facilitate complex design communication but will also encourage extensive design reflection. Utilizing this method in conjunction with $3 \mathrm{~d}$ modelling provides extensive design visualisation as well as providing an understanding of how models may be perceived within the design context.

\section{Design Reflection}

Design reflection will play a vital role in regard to how the design progression throughout the design process. Reflection based on design analysis will be applied in order to carry out appropriate design decisions, ensuring the design is constantly developed and refined. The process of continuous critical thinking will determine the resolved outcome as the design decisions will ultimately construct how the final model responds to the design challenges. Furthermore, deciding how constraints were satisfied and opportunities utilized. 


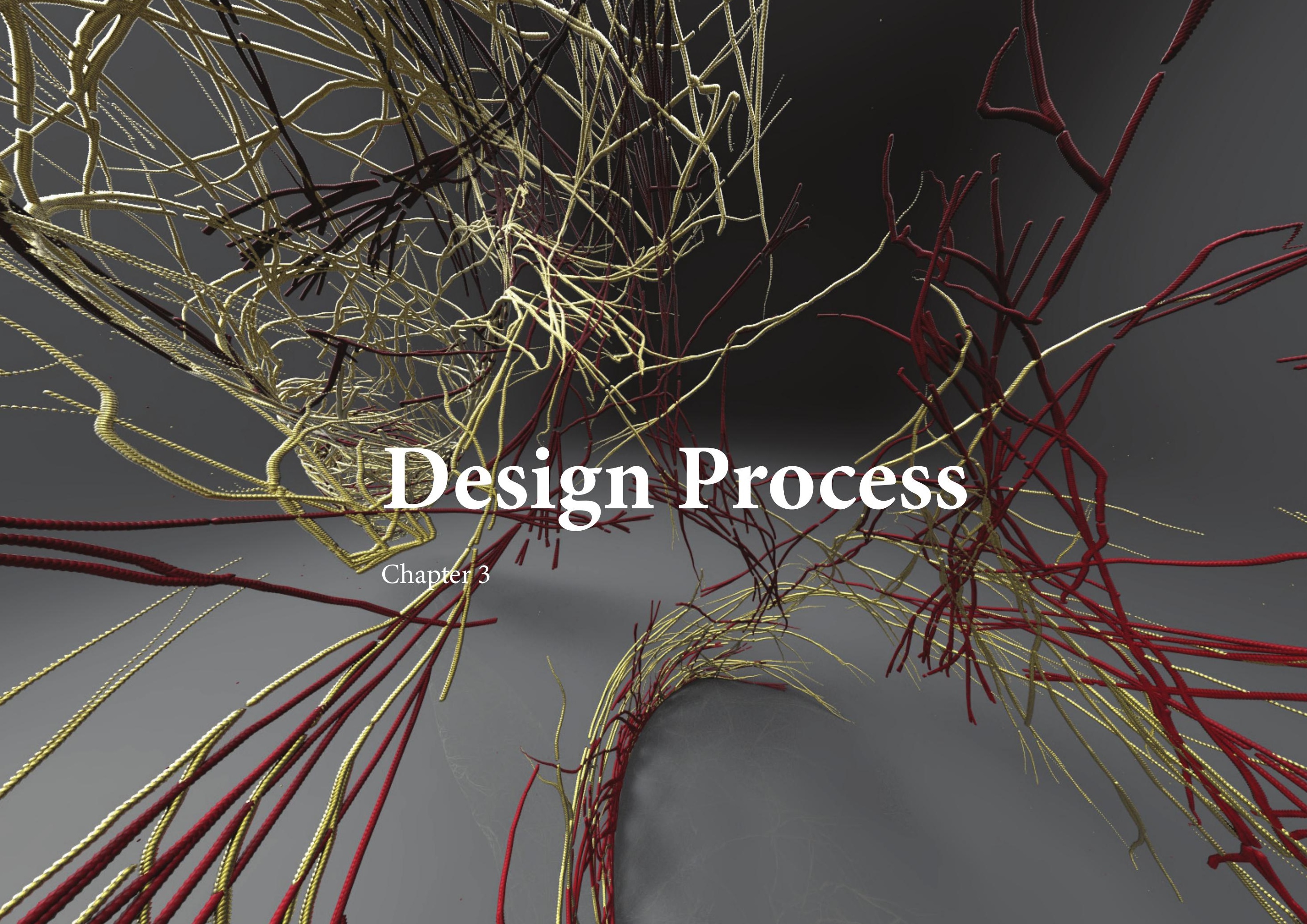



This design phase will be utilized to conjure a design approach, through continuous creative thinking. Through sketching the aim is to encourage visual thinking, exploration speculative concepts and design reflection. From these sketches a final concept will be selected and produced utilizing effective and relevant methods. 

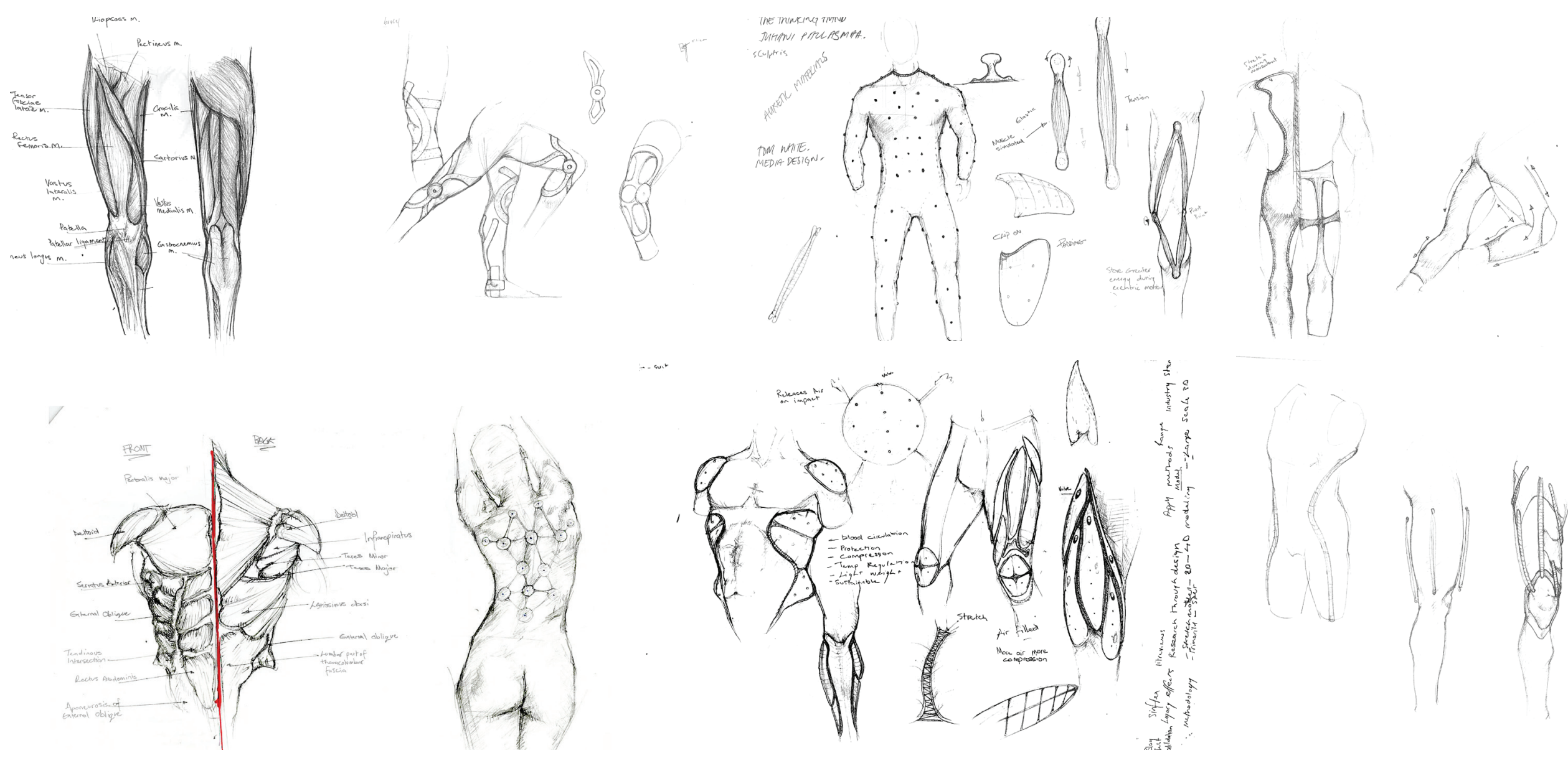

Figure 3.1

Sketches exploring human anatomy and performance enhancing wearable concepts. 

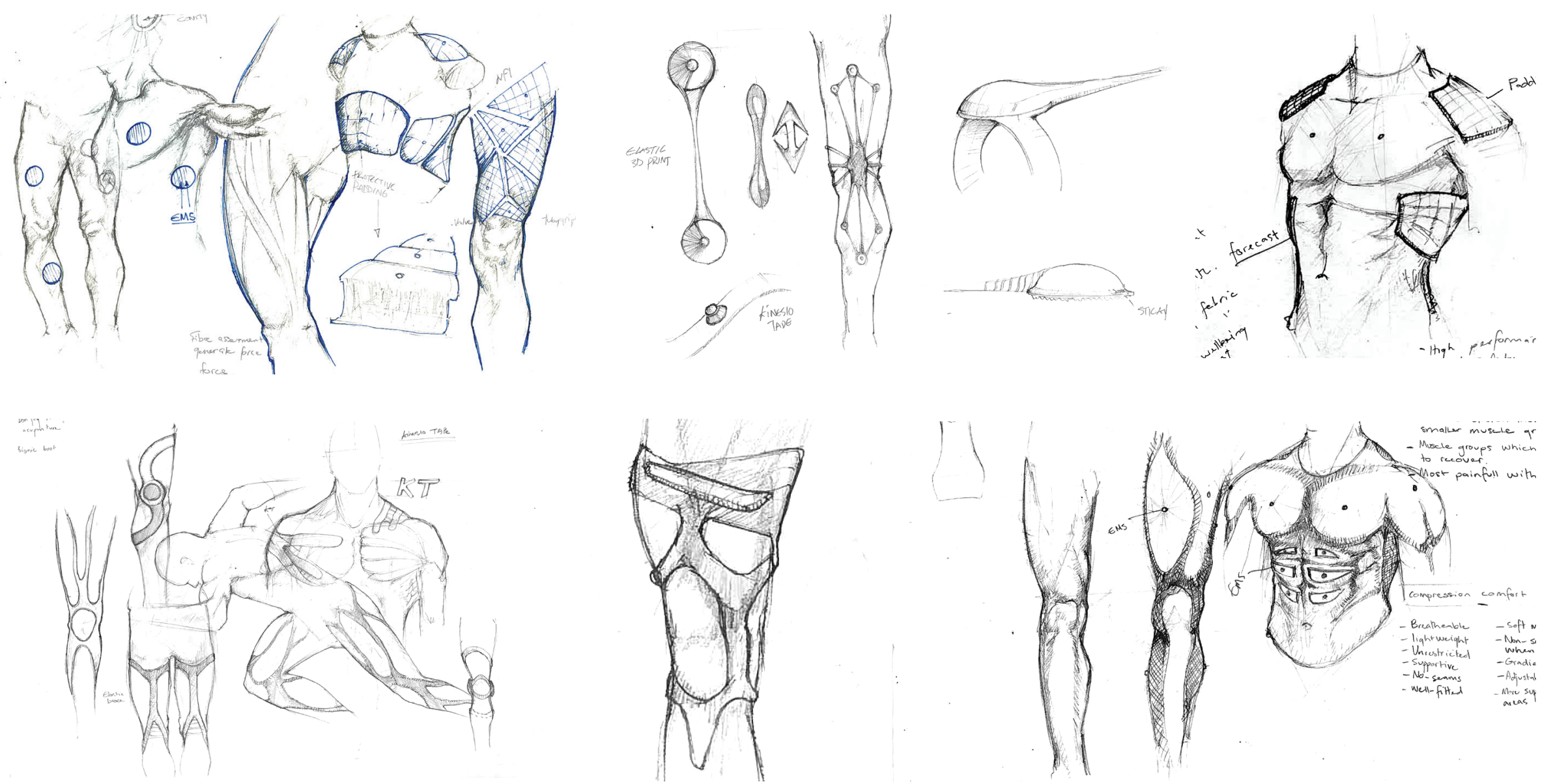

Figure 3.2

Sketches exploring possible performance enhancing strategies in regards to the human anatomy 

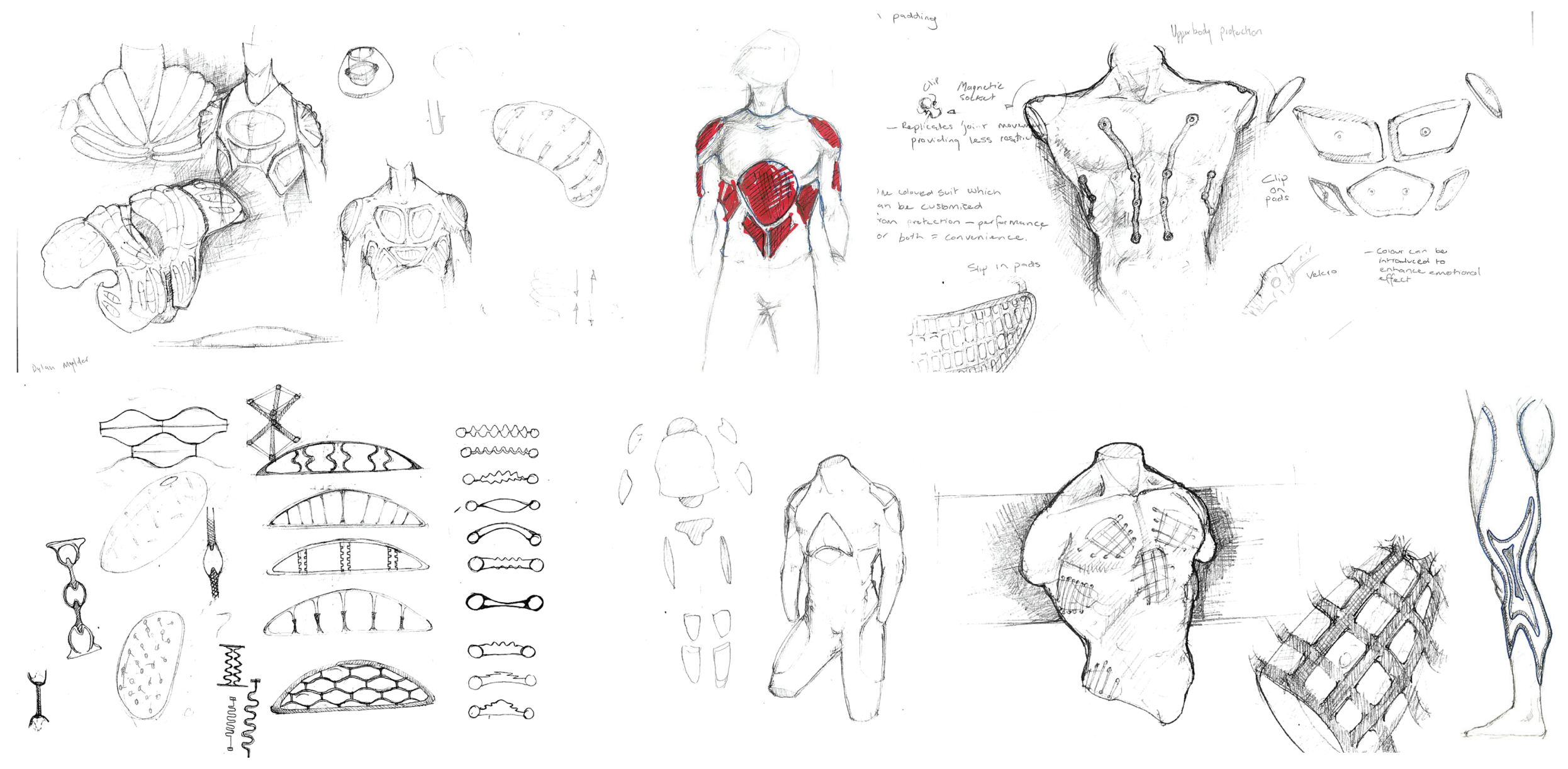

Figure 3.3

Exploring impact absorbing structures and how they can be customised to suit the body. 

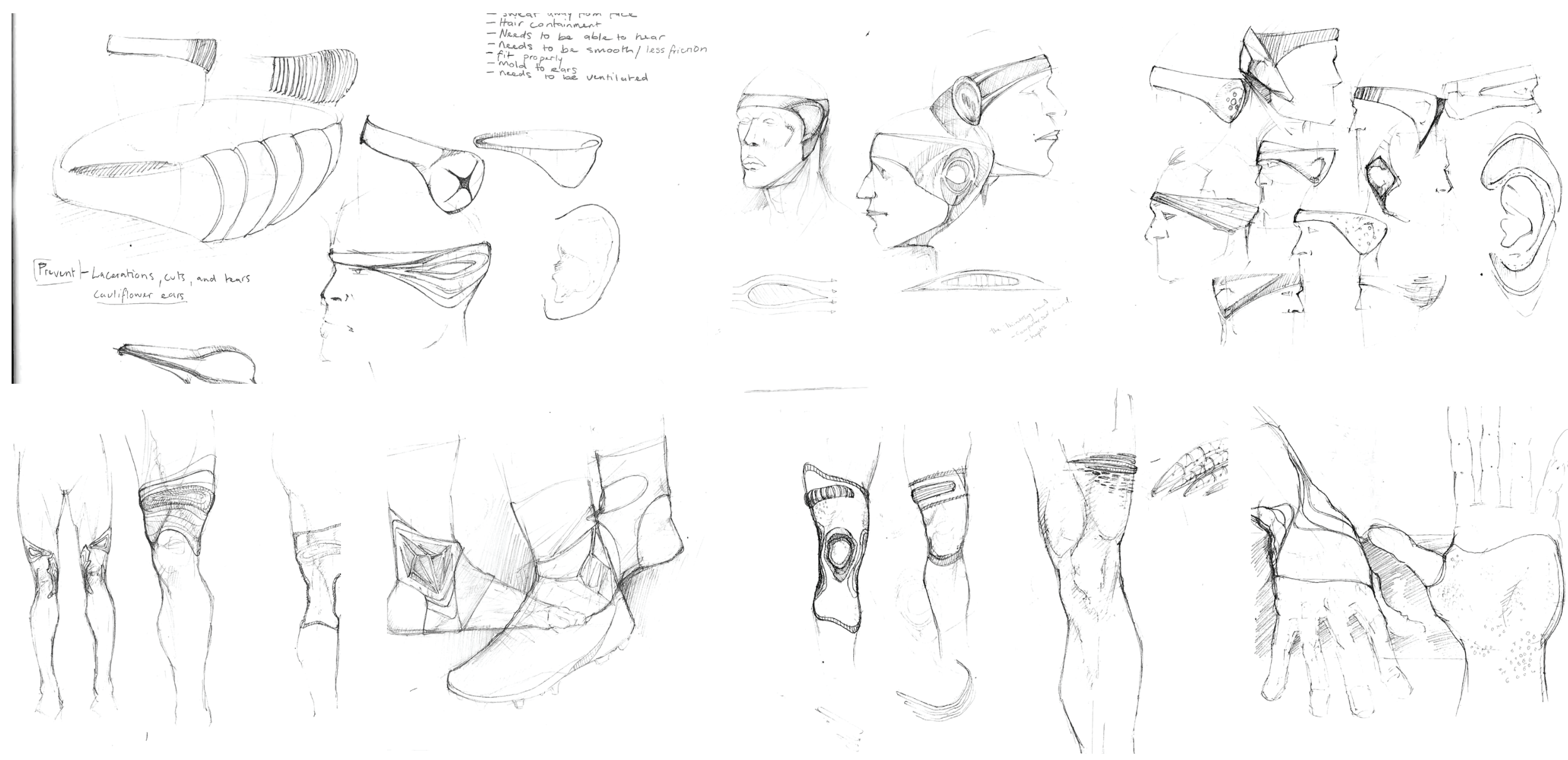

Figure 3.4

Determining how protective wear can be developed for rugby as well as enhancing sports performance 

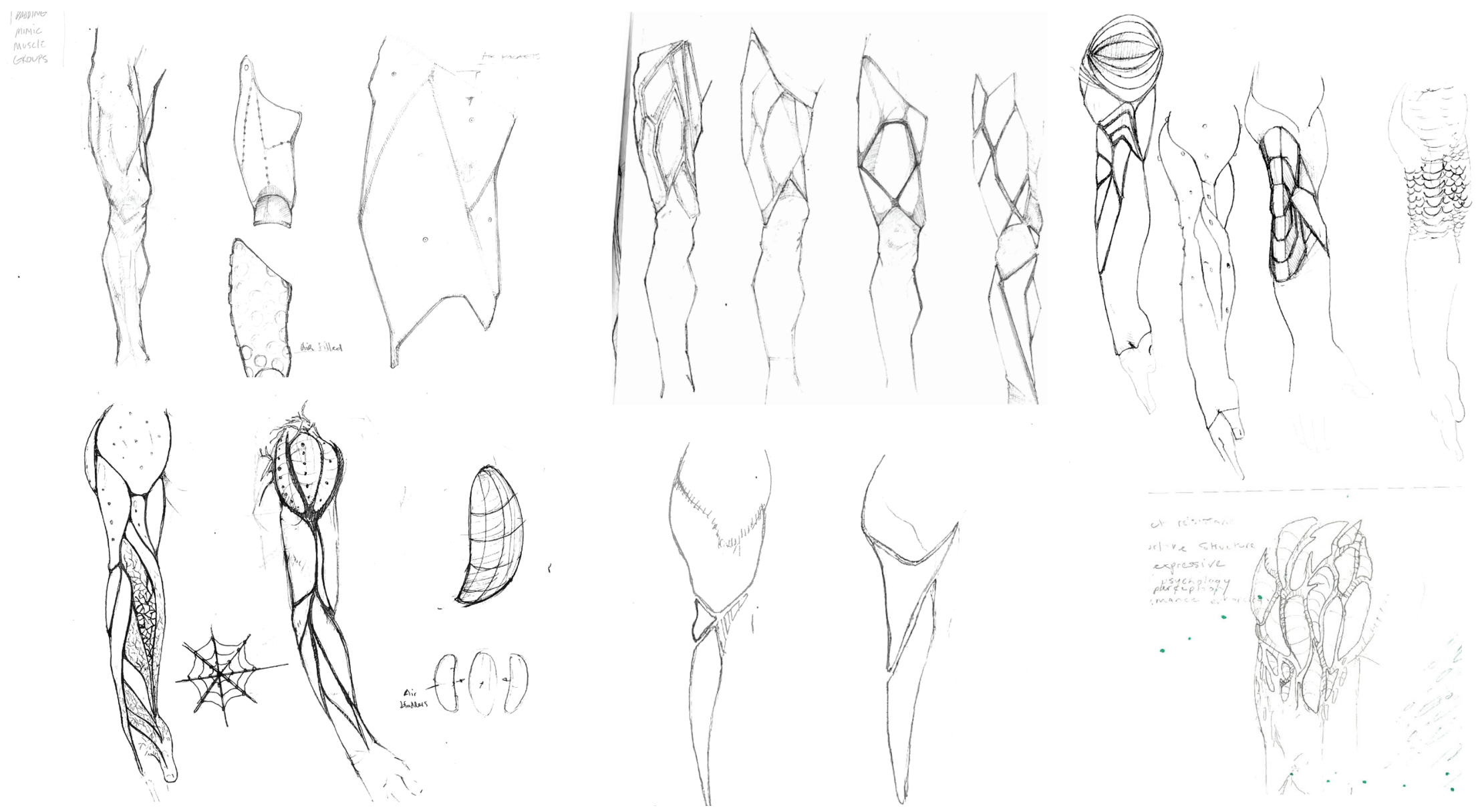

Figure 3.5

Sectioning the leg muscles based on the major muscle groups to allow for dynamic movement. 


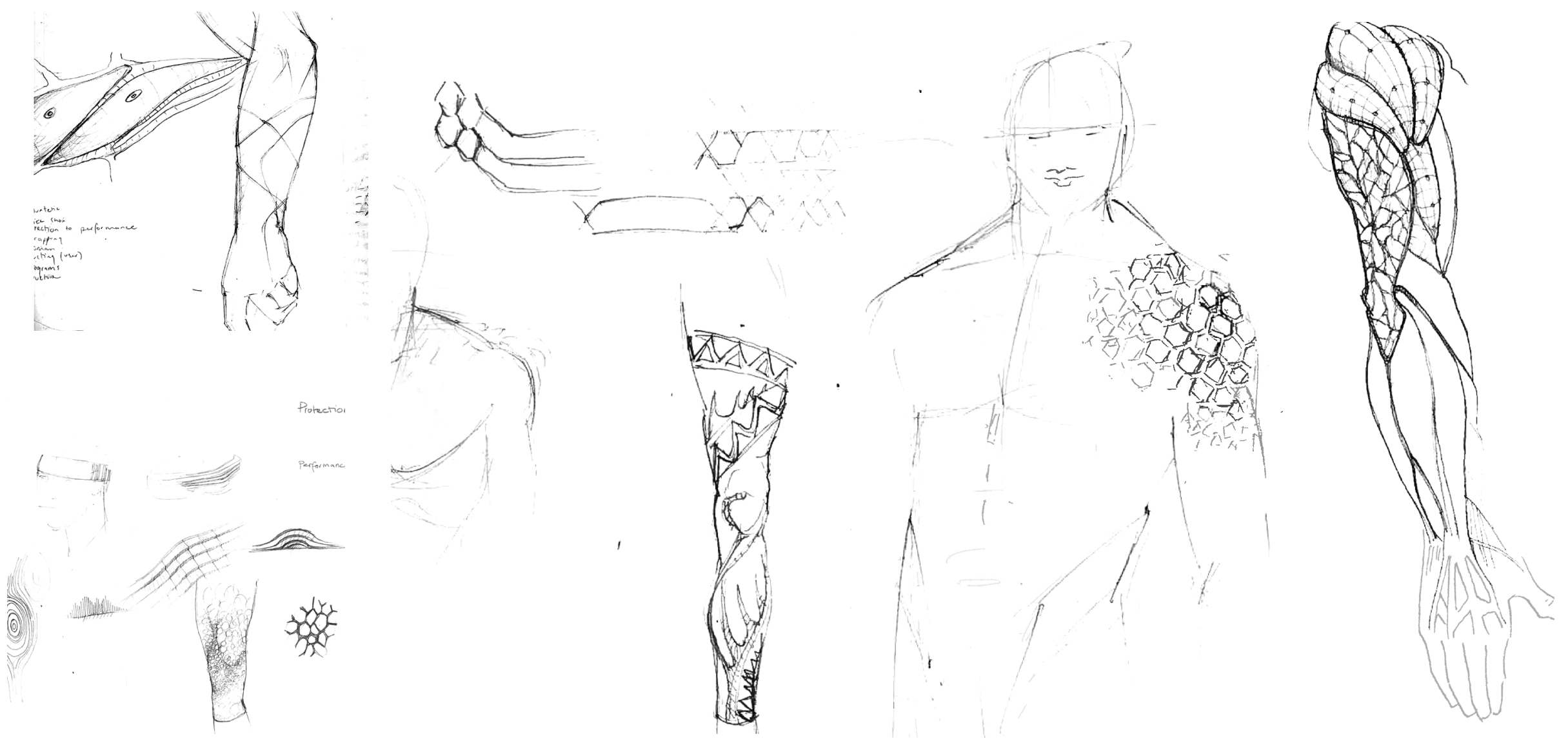

Figure 3.6

Exploring structural patterns and how they can be applied to the human form. 


\section{Design Approach}

\section{To The Roots}

Through constant iterative thinking and the exploration of multiple concepts a final concept was acquired. Giving the thesis an initial design approach and a foundation for a growing design. The design idea was constructed through looking at the root systems of trees. Roots are vital to the health and longevity of trees as all plants need water, oxygen, and nutrients. In vascular plants, the root is the organ of a plant that typically lies below the surface of the soil. However some can also be aerial or aerating, growing above ground or water. Analysing the physical and visual qualities of how these roots behave sparked an idea. Even more so when analysing how they interact with urbanised constructions such as pavements. It has been shown that if trees are too close to pavements, roots can eventually lift it because when roots encounter a paved area, its only way of entry are the gaps between the soil and pavement. This demonstrates the physical strength and intelligence of the tree root. This created inspiration to grow an organic protective system, the concept of having an aerating root system grown around a limb provided the protective investigation with a sense of direction, however speculative it may be.
Applying this concept to the human body, the cardiovascular system (veins) were analysed. Looking at how and where veins migrate inside the human body and how this can be become aerating veins on the surface of the skin. This was done by arguing that the areas where veins reside most, are more sensitive and in need of greater protection. Therefore the human veins system was utilized as a roadmap in regards to where protection is required most.

Relating this to sports, common among rugby players athletes wrap body parts and joints to reduce risk of injury and improve performance. However, this concept provides players with a customised wearable that can be worn daily, removing constant waste and purchasing of tape and bandages. Another reason why roots and veins are you is due to their symbolic meanings. They can be interpreted as the personal growth, foundation and identity of an individual. Through this the aim is to make athletes self-aware of their purpose and goals stimulating motivation and confidence which will effectively result in an enhanced performance. 


\section{Scales}

To create a protective skin that focuses more on cuts, scratches and abrasions the thesis looked at biomimicry. As discussed in the literature review, $3 \mathrm{~d}$ printing biomimicry has been done very successfully in past case studies. In this case, a design approach was taken involving analysing scales of fish, snakes etc. Looking at animals with scales, the idea was to create a second skin with qualities that can aid for sports protection. Besides having the ability to provide animals with a protective layer the structure of scales provide enough dynamic movement to move freely as shown by the snake and fish. This is essential to athletes as sports requires a vast amount of movement, disrupting any natural movements will evidently impact sports performance negatively. Furthermore, scale structures that are overlapping can also be designed to lock in or restrict movement. Providing the ability to control movements in certain directions that may harm athletes. These mechanisms can be applied to to limit over extending or any irregular movements. Using overlapping for protection has been used for centuries as shown throughout ancient roman history. Although primitive, this technique served a purpose reliable enough to ride into war with.

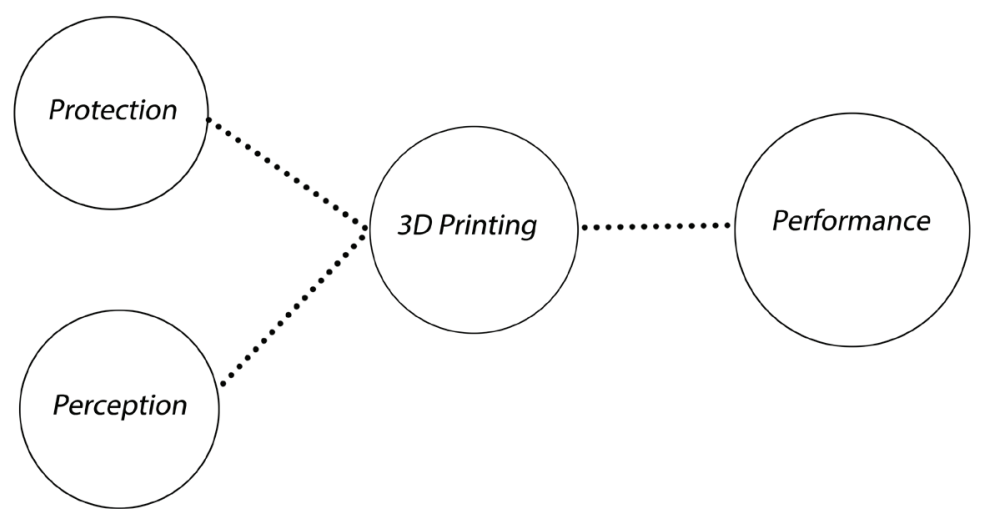

Figure 3.7

Diagram demonstrating the strategy for enhancing sports performance. 


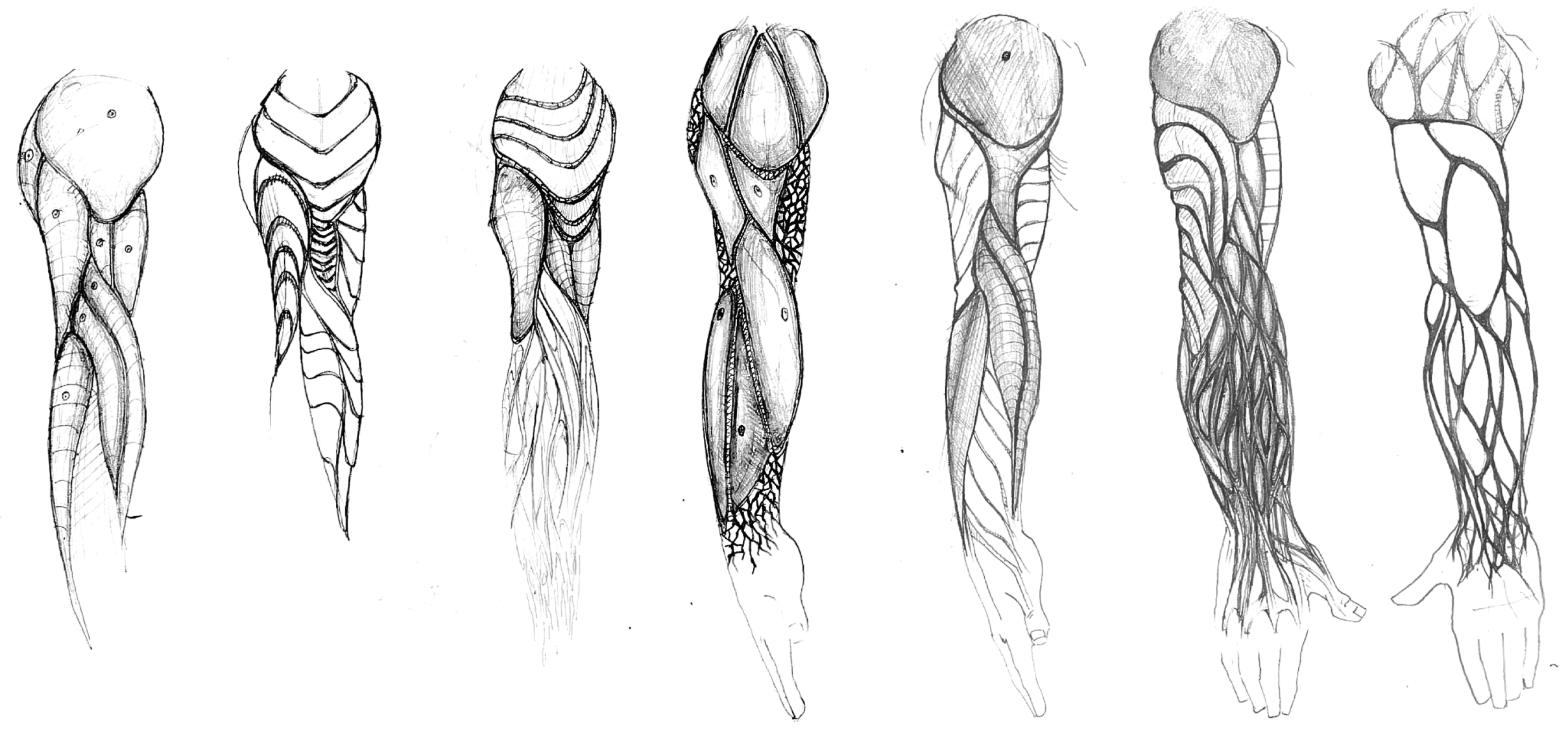

Figure 3.8

Exploring protective compositions based on the anatomy of the human arm. 

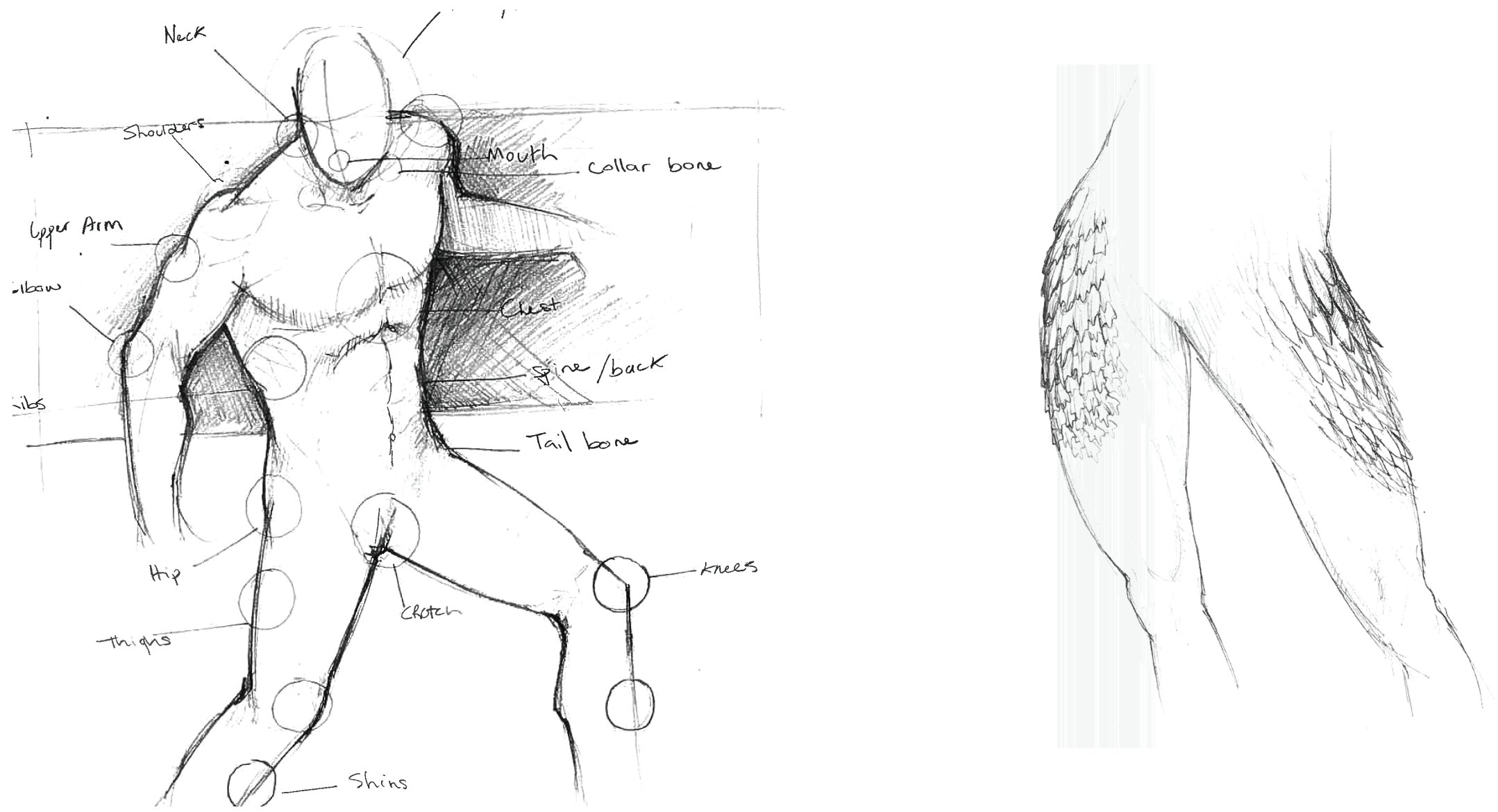

Figure 3.9

Determining which areas on on the body needs protection based on vulnerability. 


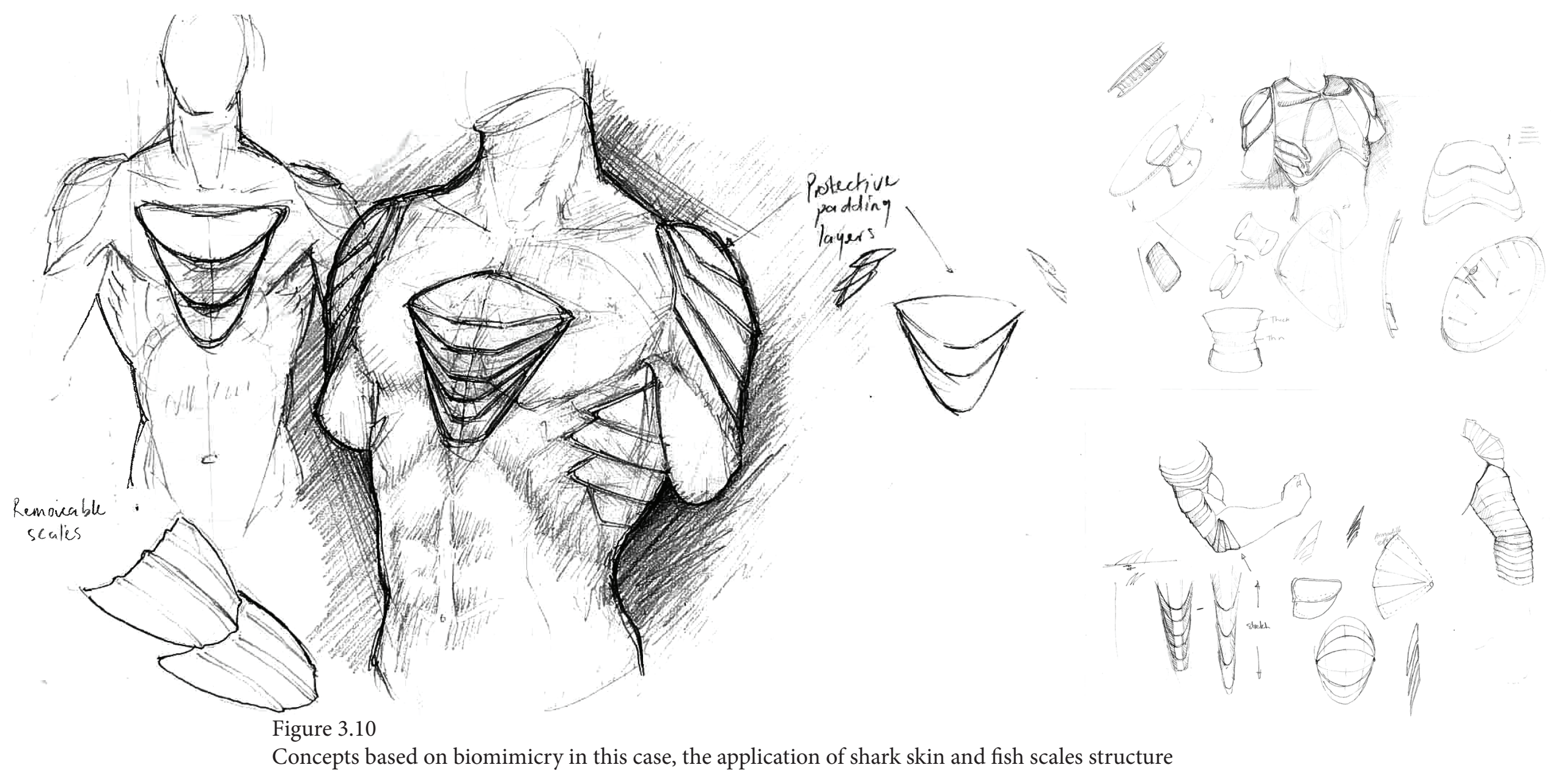



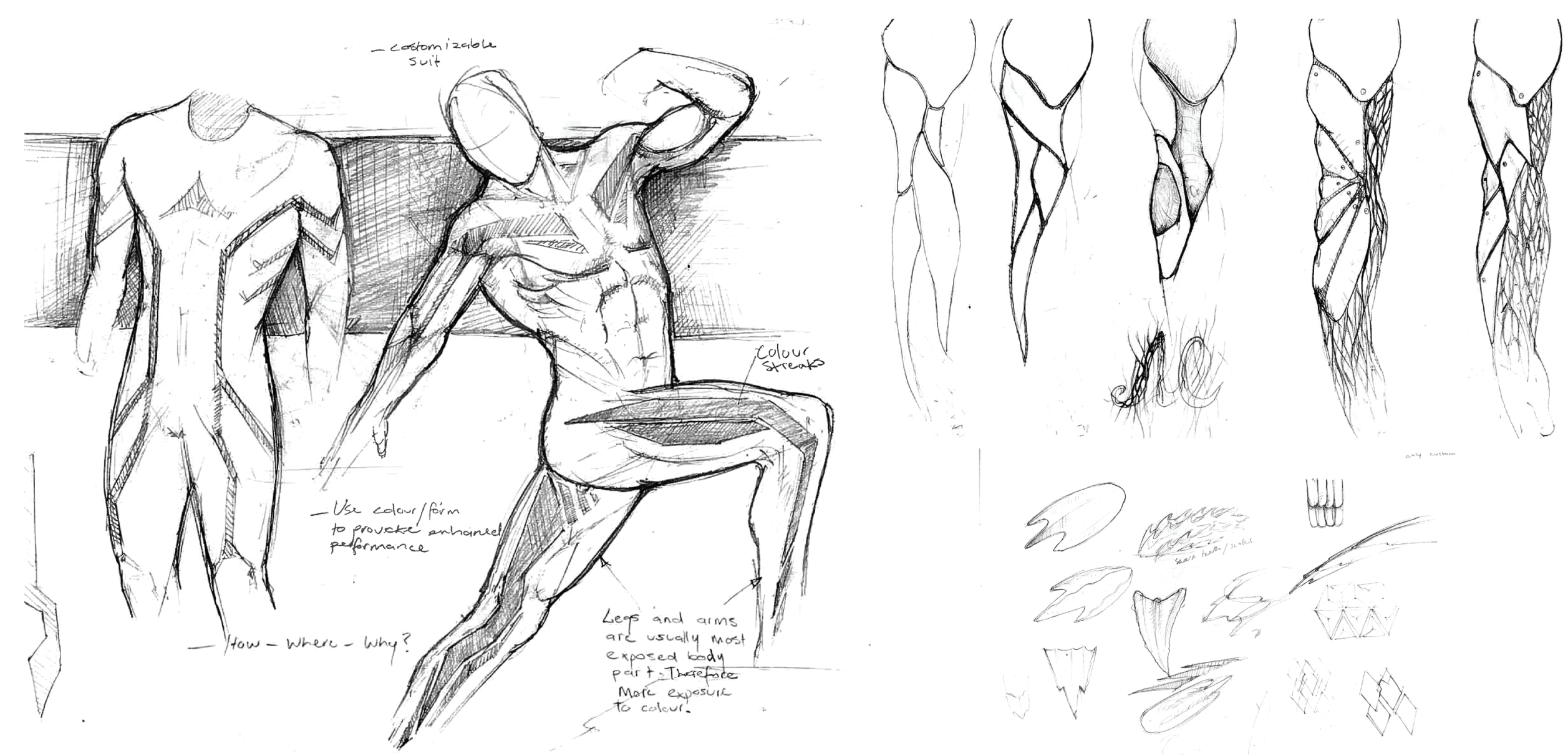

Figure 3.11

Analysing how sports perception can be introduced through form and colour.

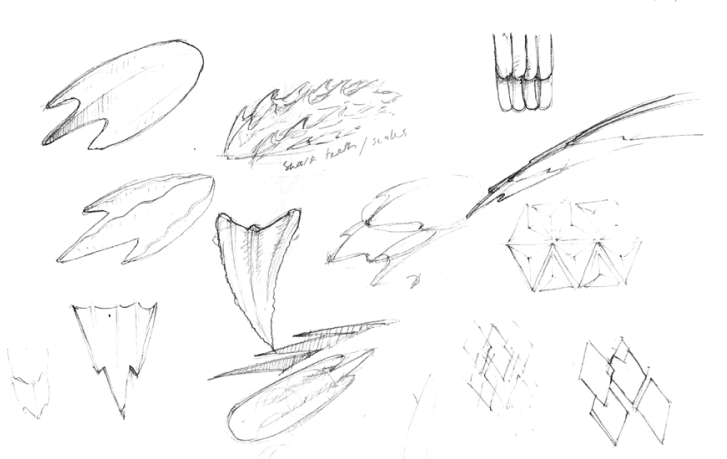


Exploring 3D Printing Part One

Chapter 4

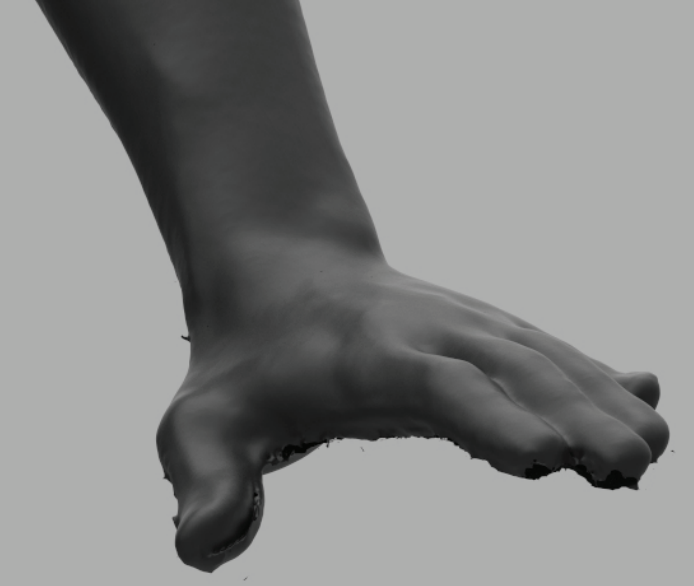


This phase covers the initial $3 \mathrm{~d}$ printing experimentation, discussing how the $3 \mathrm{~d}$ printing process developed from the first $3 \mathrm{~d}$ printed samples. Furthermore, selecting relevant findings and defining how they can be exploited and applied to facilitate design development. 


\section{Raft and Support}

Rafts are generally the foundation of an average fused deposition modeling (FDM) 3d print, it's a horizontal latticework made of filament that is located underneath the printed part. Raft is used as the groundwork of a $3 \mathrm{~d}$ print, primarily utilized to help create better bed adhesion. These bottom raft layers are printed slowly and thick to ensure a strong bond to the build platform, reducing any unwanted movement during the $3 \mathrm{~d}$ printing process. After a print is completed the raft can then be removed leaving a good surface finish on the bottom of the print.

When using FDM $3 \mathrm{~d}$ printing, each layer is printed as a set of melted filament on the build platform surface. The layered threads produced adhere to the threads below and around it to allow for complex models to be built. Each FDM thread is printed with a slight offset relative to the previous layer. Through this process models which contain angles of 45 degrees can be built. However, when the FDM $3 \mathrm{~d}$ printer encounters angles greater than 45 degrees, sagging can occur. To overcome this problem support material is required beneath the overhang in order to hold the model up and prevent any distortion.

Although, one exception to this rule is a technique called bridging. This is where hot material is stretched short distances between two points, given they are within $5 \mathrm{~mm}$ of each other. A simple way to understand this is considering the letter $\mathrm{Y}, \mathrm{H}$ and $\mathrm{T}$. The arms of the letter $\mathrm{Y}$ can be printed easily as they extend at 45 degrees or less, therefore no support is required. On the other hand, the Letter $\mathrm{H}$ does require support due to its centre bridge, unless the given distance is $5 \mathrm{~mm}$ or less.
Furthermore, letter $\mathrm{T}$ requires support for its extended arms as there are nothing for it to be printed on. Therefore, when designing $3 \mathrm{~d}$ models, consideration as to how they will be printed structurally will need to be required In this case, creating designs with a flat foundation will avoid the need for raft as it can be placed on the print bed without any overhanging.

A downfall when using support material is the added requirement of post processing. Removing support especially if created in small, intricate features of the model can be complex. To decrease risk of damage each support element needs to be removed using needle-nose pliers. Ontop of this. sanding may be required for smoothing the surface to the desired quality. Another problem that arises is the need for extra material, creating more work and additional costs. However, by placing support in the correct and or specific locations, the use of support material can be reduced effeciently.

The Initial the $3 \mathrm{~d}$ prints in this thesis will be done using no raft or support material because the process of removing raft and support material may prove extremely difficult without causing any damage to the model or textile. This because the support and raft will fused to the textiles during the $3 \mathrm{~d}$ printed process, removing the $3 \mathrm{~d}$ print without damaging or dethatching the model will be near impossible. $3 \mathrm{~d}$ printing without raft or support material means making sure all the designs does not require any additional support and that each model have a flat base to ensure effective bonding. 


\section{Contouring}

As this thesis focuses on how $3 \mathrm{~d}$ printing can aid sports performance and protection, research through design was carried out. Analysing and discovering how $3 \mathrm{~d}$ printing material interacts with textiles.. Using an FDM Up Box 3d printer along with PLA, TPU and ABS filament a series of $3 \mathrm{~d}$ printing experiments were done. Initially, these involved investigating how printed contour lines may create or distort a form when fused to the textiles. On top of this how the stretch direction and degree of stretch may alter or distort the outcome.

During the experimentation process a range of materials were used. Textiles consisted of nylon and lycra mixed with cotton. The reason behind using these elastic materials were to allow flexibility when stretching over the print bed so that the textile threads are opened allowing the base filament layers to fuse more effectively. Moreover, ensuring that the nozzle height is adequate because if too close the material can be burnt and ripped. If the nozzle is too far, the filament may not fuse properly and instead sit on top of the fabric.

Using materials such as nylon will allow the wearable to mould to the body and ensure a tight, secure fit. In terms of the filament material, flexible TPU were used to prevent loss of dynamic movement, as well as providing greater comfort for the users due to its soft qualities. 
By stretching the material prior to $3 \mathrm{~d}$ printing, findings show when the fabric is removed tension is preserved by the $3 \mathrm{~d}$ printed filament .Therefore, if a straight line is to be printed in the same direction of the stretch, it will become arced as a result of the stretch tension. Through using this technique, and controlling where and how much the textiles are stretched the $3 \mathrm{~d}$ printed design can possibly provide a better fit to the contours of the intended body part. However to allow for this curvature to happen, the textiles needs to have enough tensile strength to bend the $3 \mathrm{~d}$ print. This can be assisted by $3 \mathrm{~d}$ printing flexible filament and reducing the thickness to allow for easy bending. If the $3 \mathrm{~d}$ print is too thick and heavy the tensile strength will have little to no effect on the final outcome. Furthermore, when printing on the textiles whilst fully stretched this removes its capability to be stretched further after the printing process has taken place. This occurs because the fabric becomes locked by the filament and cannot return to its original state unless the filament is removed. This means the form will maintain its final outcome but will become reduced in terms of elasticity.
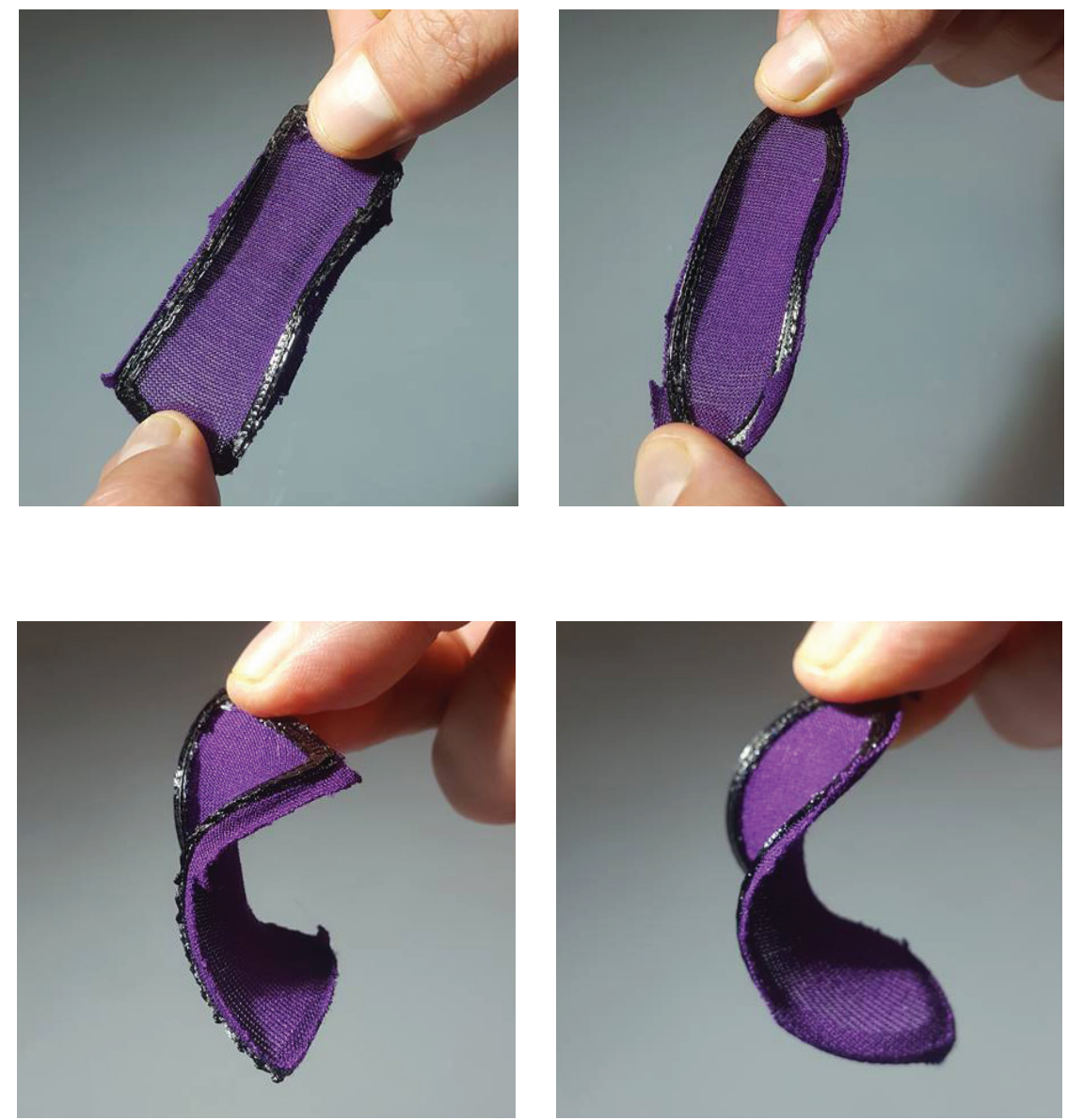

Figure 4.1

Photographs showcasing how textile tension can alter the three dimensional form of the test prints. 

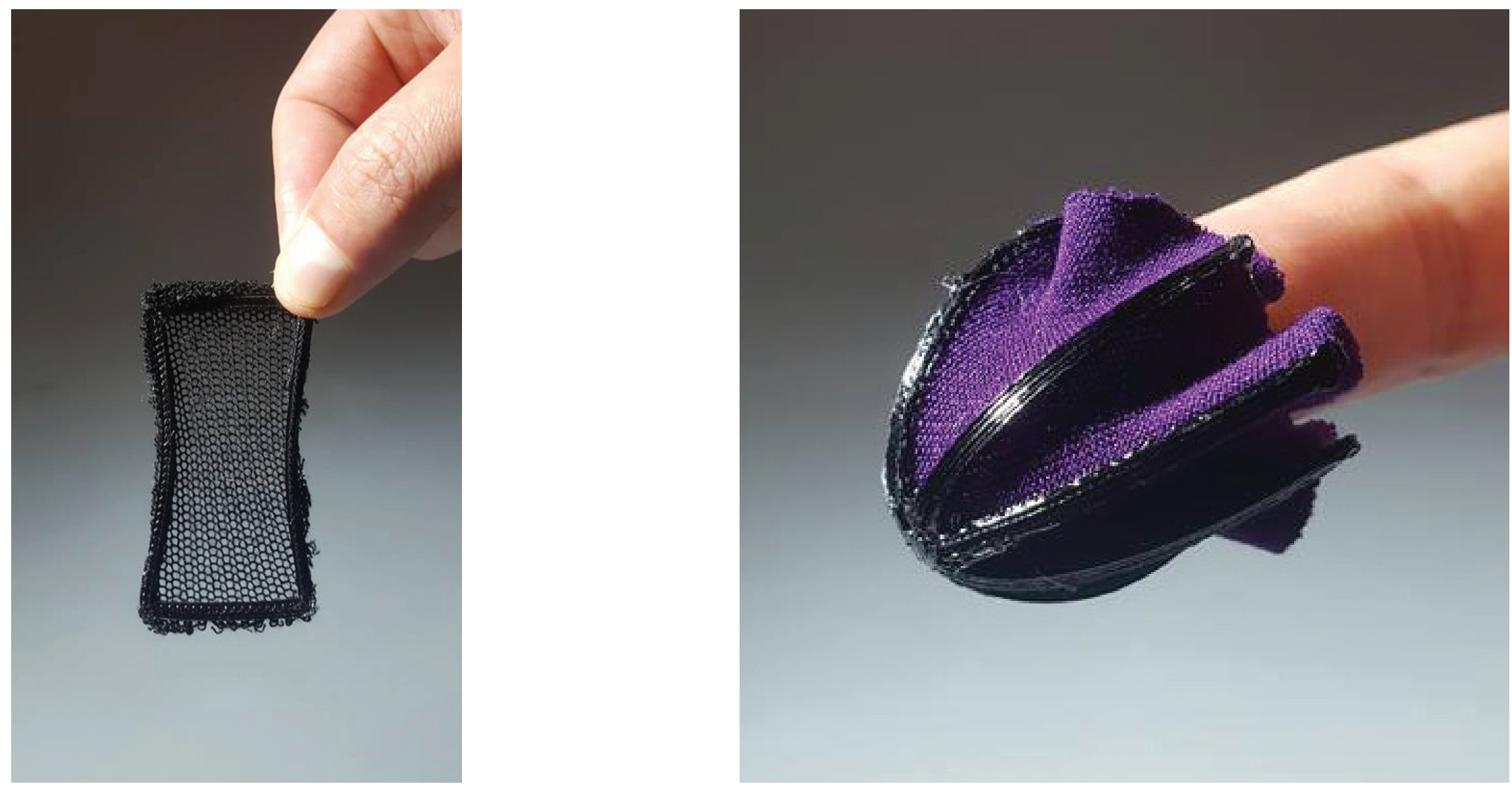

Figure 4.2

Demonstrating the contrast between textiles with low tensile strength percentage in comparison to that of a high percentage. 

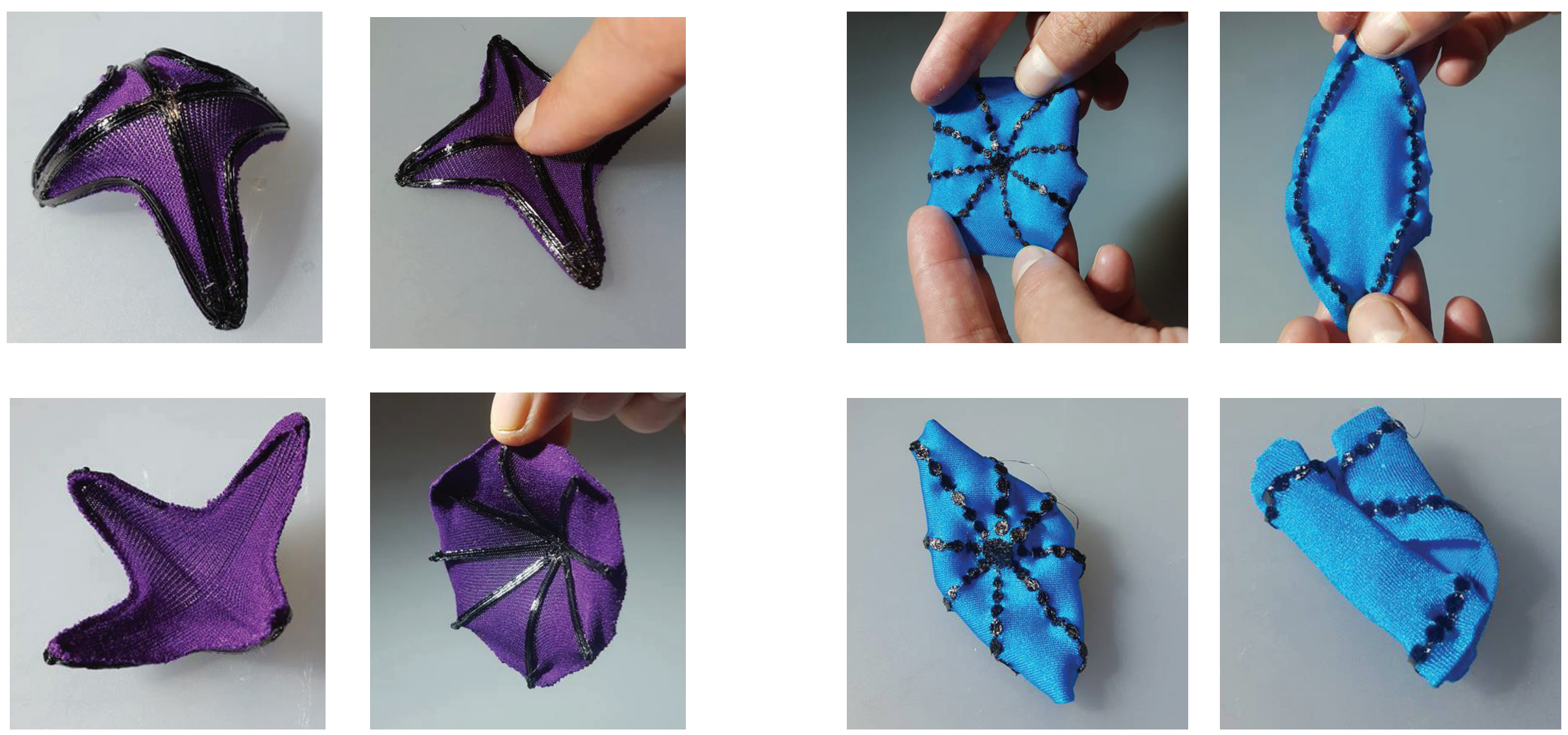

Figure 4.3

Photographs demonstrating the effect of textile tension in conjunction with the $3 \mathrm{~d}$ printed contour lines(left) and dotted lines (right). 

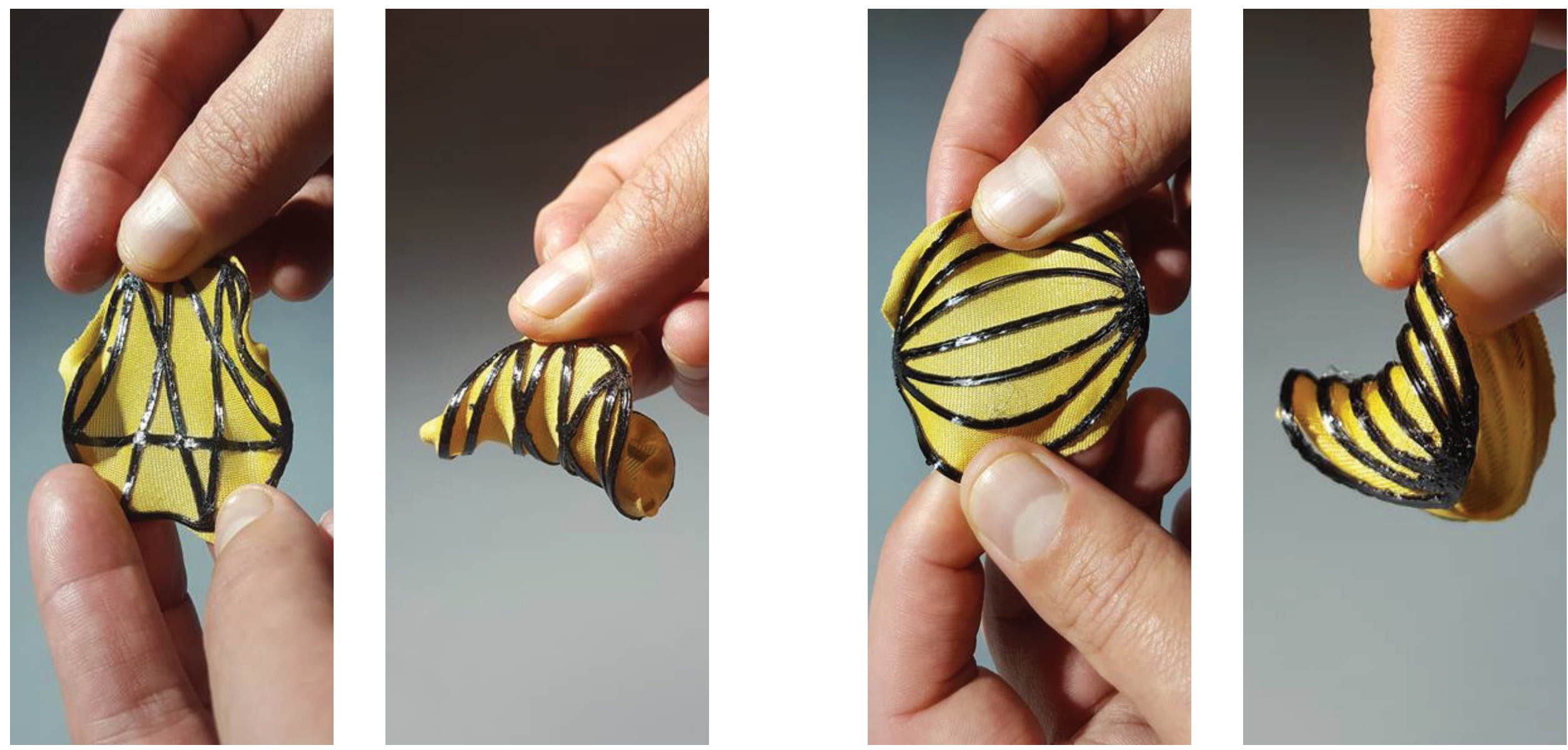

Figure 4.4

Analysing how various contour lines can create different outcomes. 

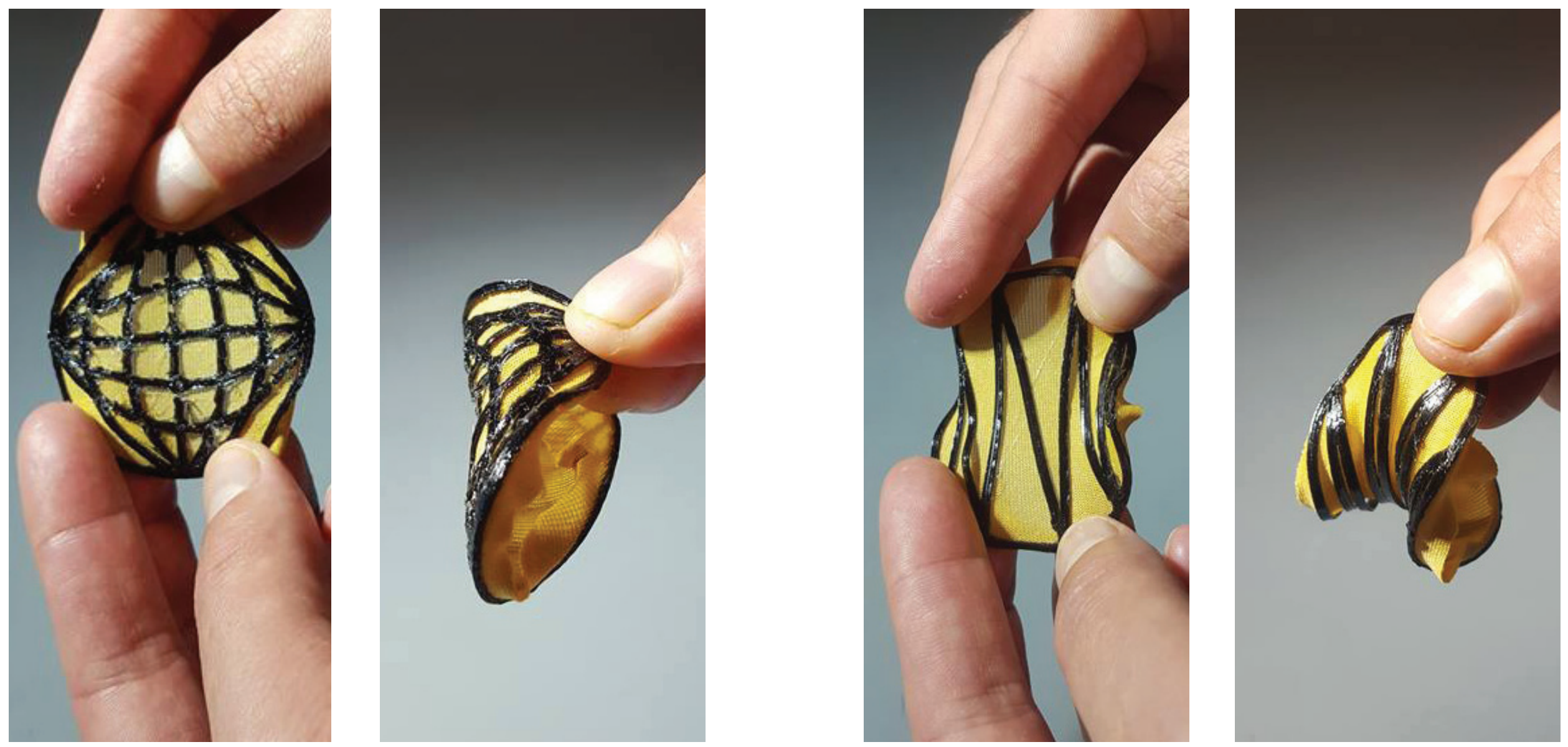

Figure 4.5

Analysing how various contour lines can create different outcomes. 


\section{Layering}
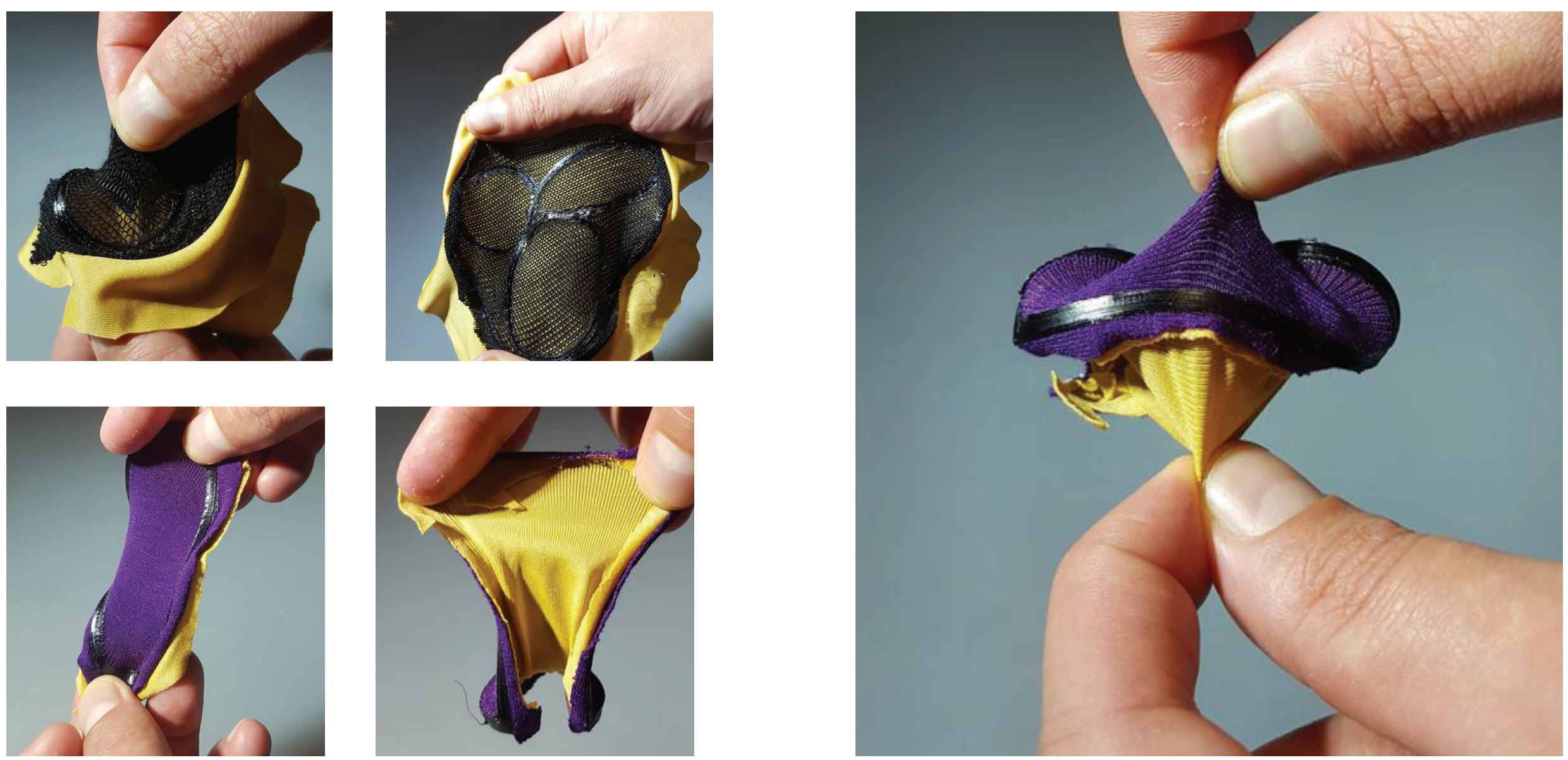

Figure 4.6

Demonstrating the adhesive strength of the layered $3 \mathrm{~d}$ printed samples. 


\section{Relfection}

By stretching the material prior to $3 \mathrm{~d}$ printing, findings show when the fabric is removed tension is preserved by the $3 \mathrm{~d}$ printed filament .Therefore, if a straight line is to be printed in the same direction of the stretch, it will become arced as a result of the stretch tension. Through using this technique, and controlling where and how much the textiles are stretched the $3 \mathrm{~d}$ printed design can possibly provide a better fit to the contours of the intended body part. However to allow for this curvature to happen, the textiles needs to have enough tensile strength to bend the $3 \mathrm{~d}$ print. This can be assisted by $3 \mathrm{~d}$ printing flexible filament and reducing the thickness to allow for easy bending. If the $3 \mathrm{~d}$ print is too thick and heavy the tensile strength will have little to no effect on the final outcome. Furthermore, when printing on the textiles whilst fully stretched this removes its capability to be stretched further after the printing process has taken place. This occurs because the fabric becomes locked by the filament and cannot return to its original state unless the filament is removed. This means the form will maintain its final outcome but will become reduced in terms of elasticity. Furthermore, this technique was repeated using dotted lines, but test samples were not as successful with preserving tension, therefore test samples were less distorted.

The combination of using multiple textiles were explored, analysing the adhesive strength of the $3 \mathrm{~d}$ printing filament as well as which materials assist effective bonding. From the $3 \mathrm{~d}$ printed samples, findings showed that textiles with a low thread count performs better. This is because of the increased space between each thread, allowing the plastic to fuse effectively with the textiles. 


\section{Scale Skin}

To create the scales through $3 \mathrm{~d}$ printing for the use of protection requires creative innovative design thinking. Initially, scale samples were designed using $3 \mathrm{~d}$ modeling software Fusion 360 and Solidworks. Furthermore, models were printed using $3 \mathrm{~d}$ printing software UP Studio. Creating these physical samples allowed for greater design thinking and evaluation based on visual and physical behavioural qualities. Therefore, using the appropriate $3 \mathrm{~d}$ printed filament and the textiles were a necessity for a functional design. By $3 \mathrm{~d}$ printing the scale samples the intent was to investigate its design effectiveness. Using a range of samples, the dynamic of the scale structures were analysed in combination with textiles. With the aim of determining how and if these samples can later become a somewhat legitimate approach to designing a protective skin. 

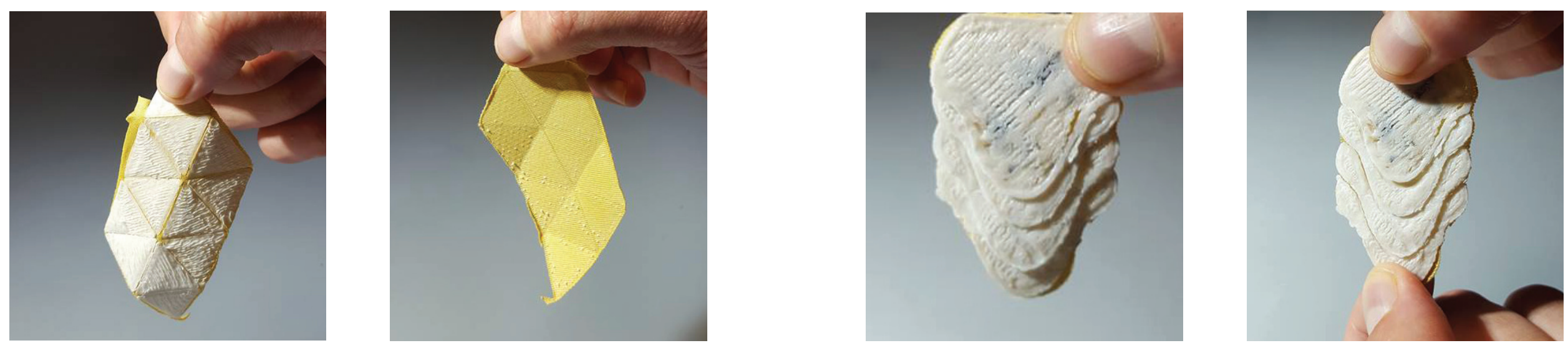
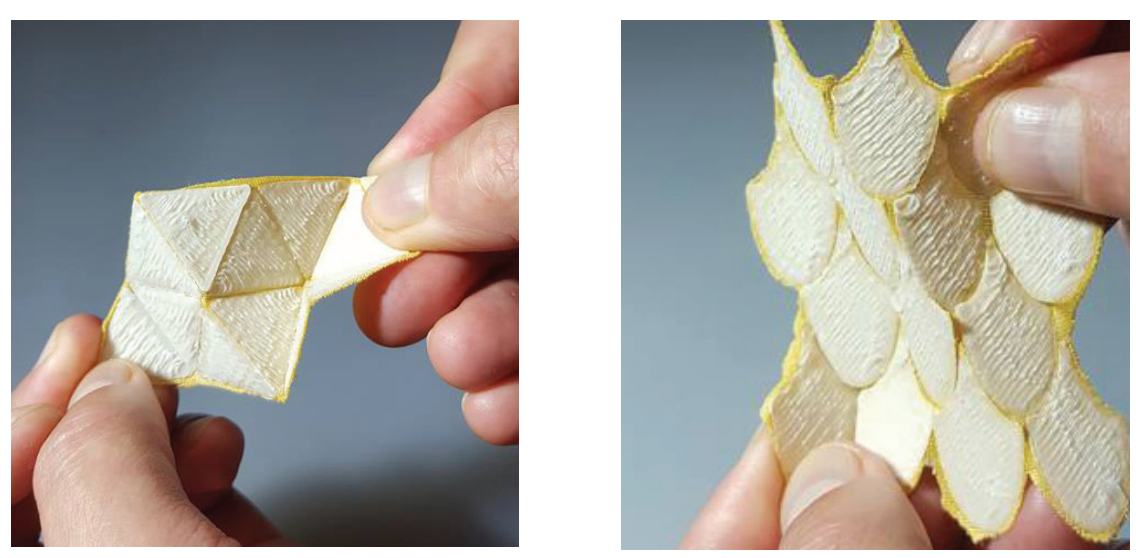

Figure 4.7

Demonstrating the dynamic qualities of the initial origami and fish scale test prints.
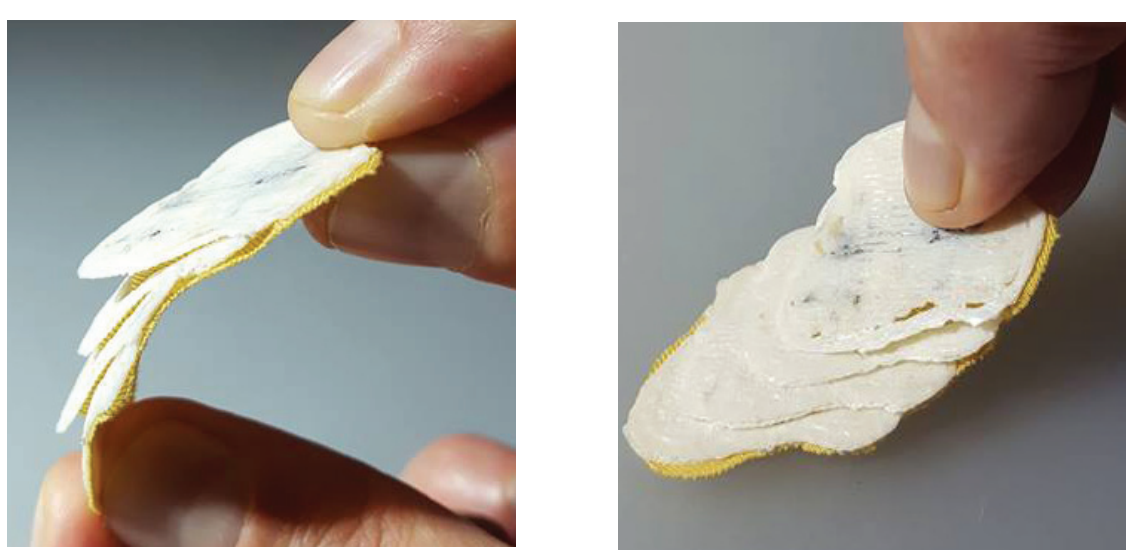

Figure 4.8

Overlapping fish scale test print dynamic sample. 

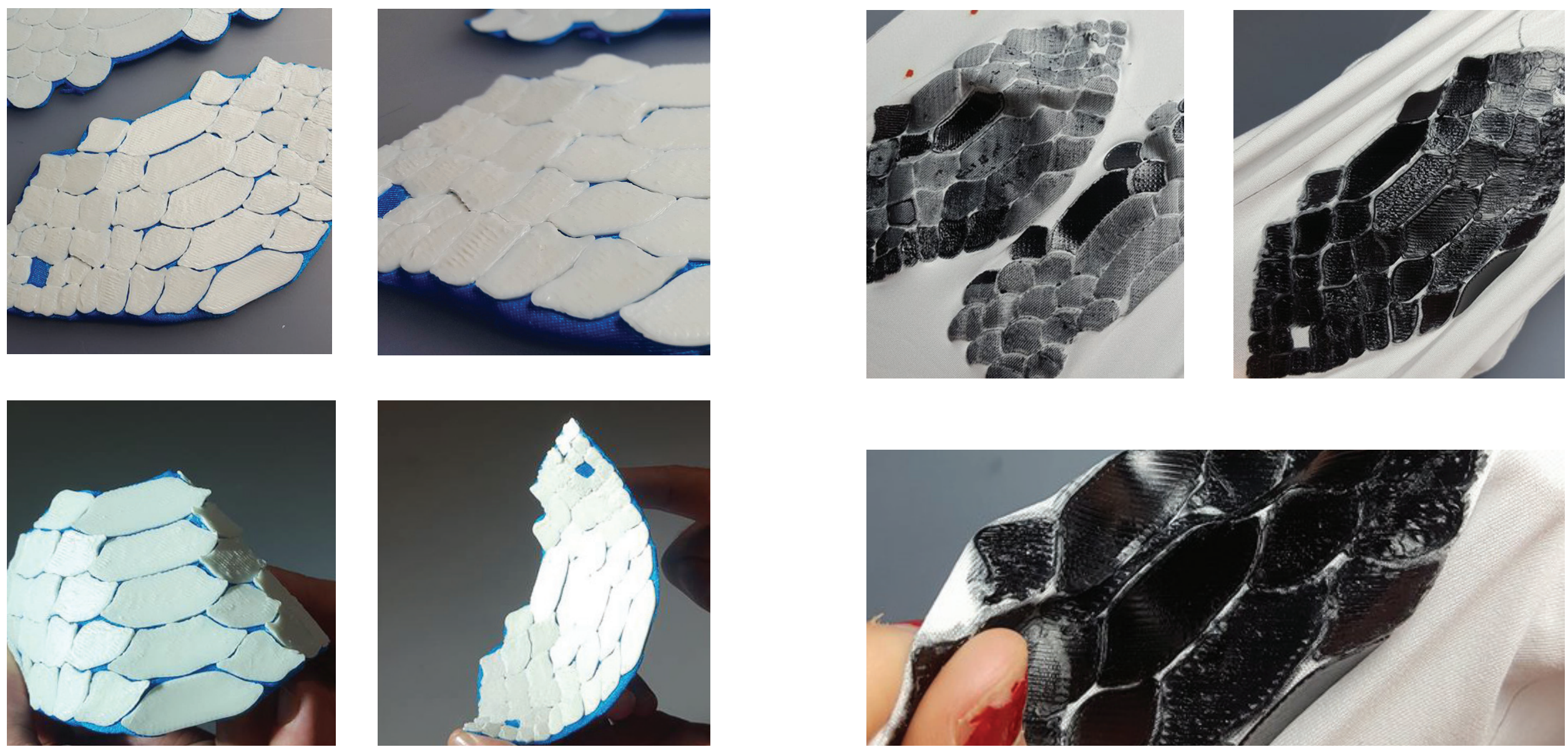

Figure 4.9

Scale configuration based on snake skin, demonstrating the locking possibilities of using a rigid $3 \mathrm{~d}$ printing filament (left), in comparison with a flexible TPU filament (right). 


\section{Stretch Test}
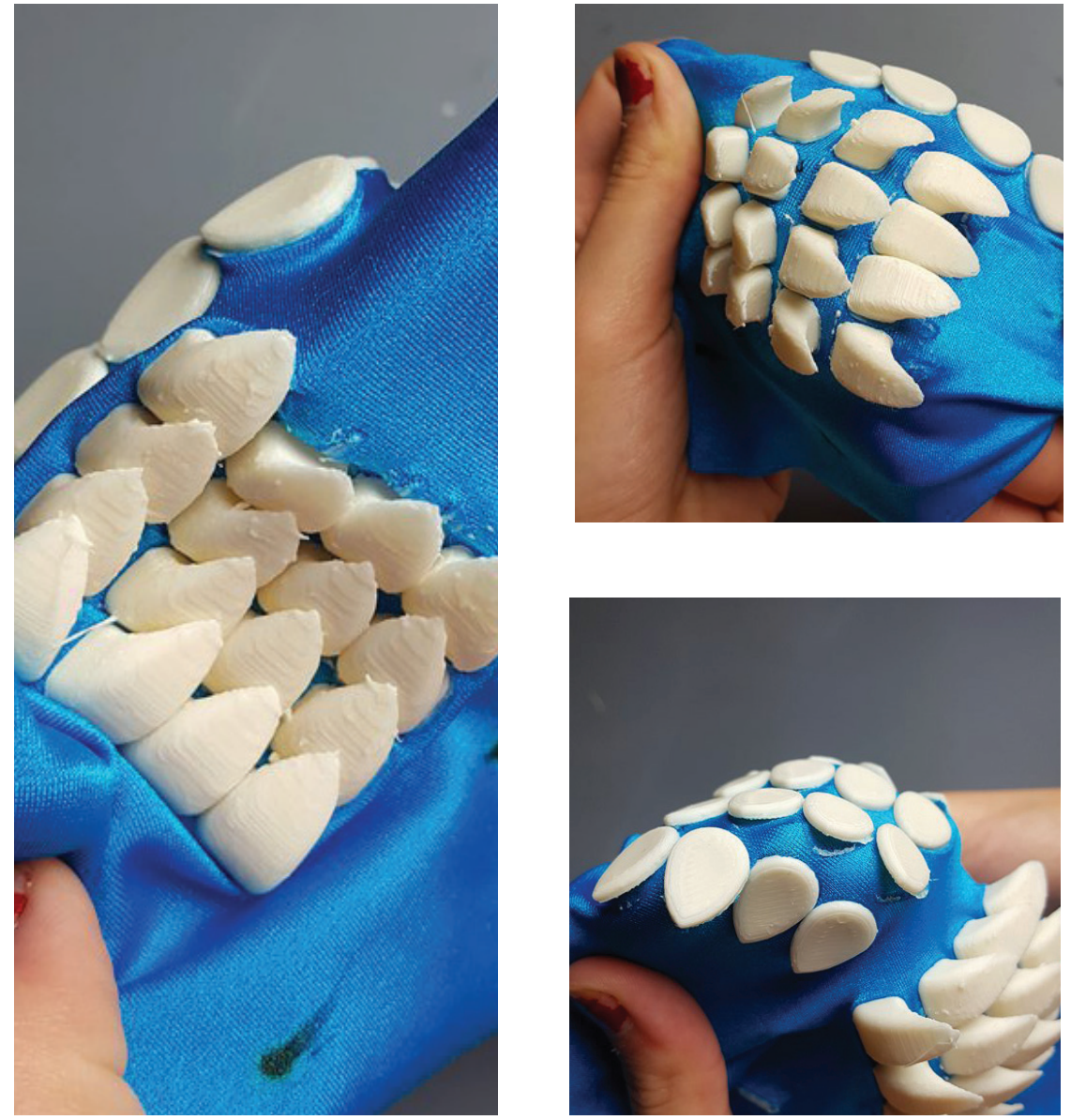

Figure 4.10

Dynamic of overlapping scales, locking to restrict motion.
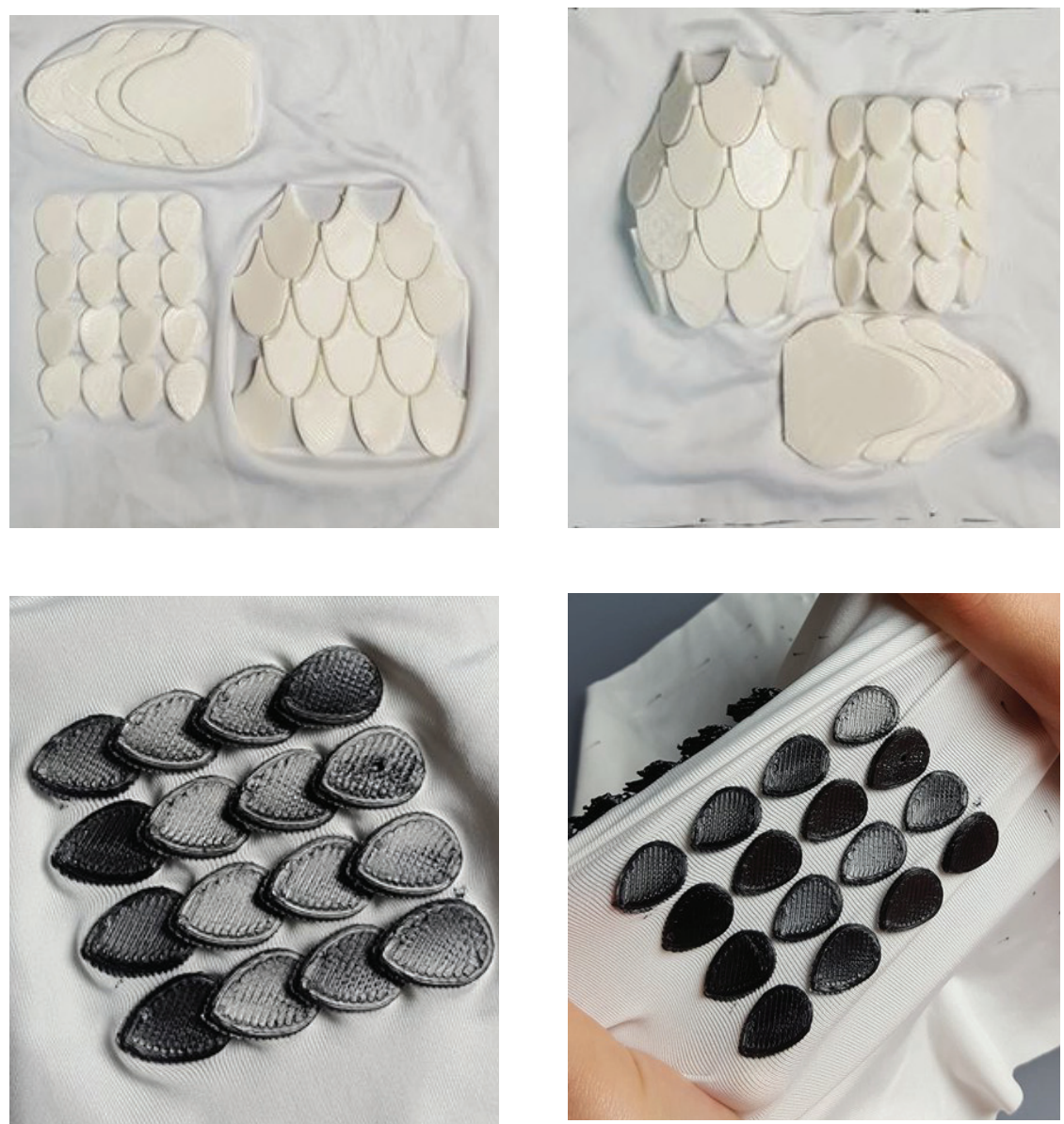

Figure 4.11

Demonstrating how stretching before $3 \mathrm{~d}$ printing can provide overlapping of scales. 


\section{Contour Scales}

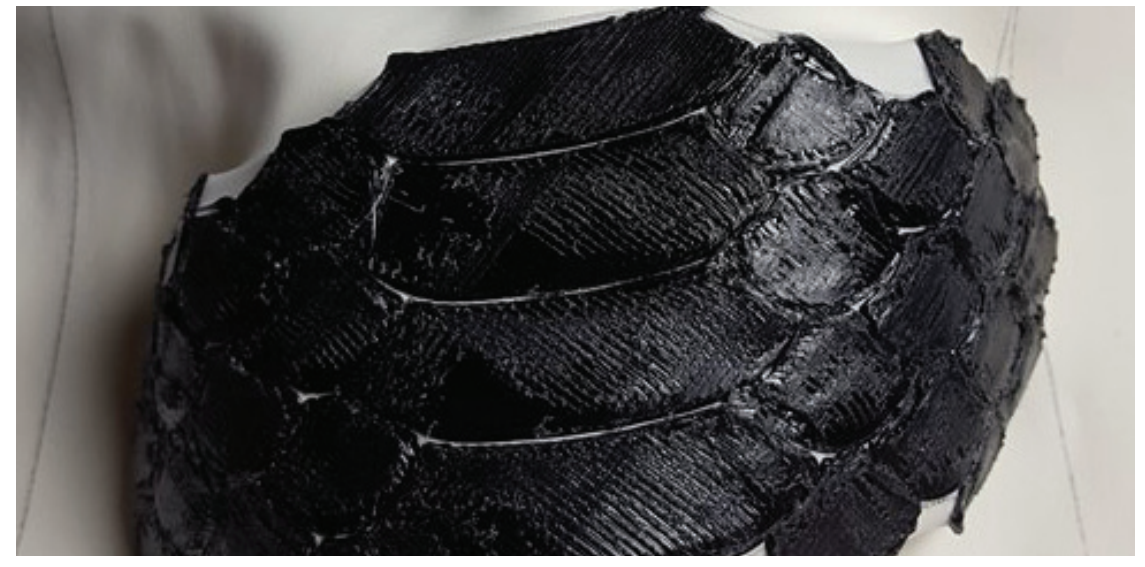

Figure 4.12

Post $3 \mathrm{~d}$ printing, contouring based on the direction of tension.
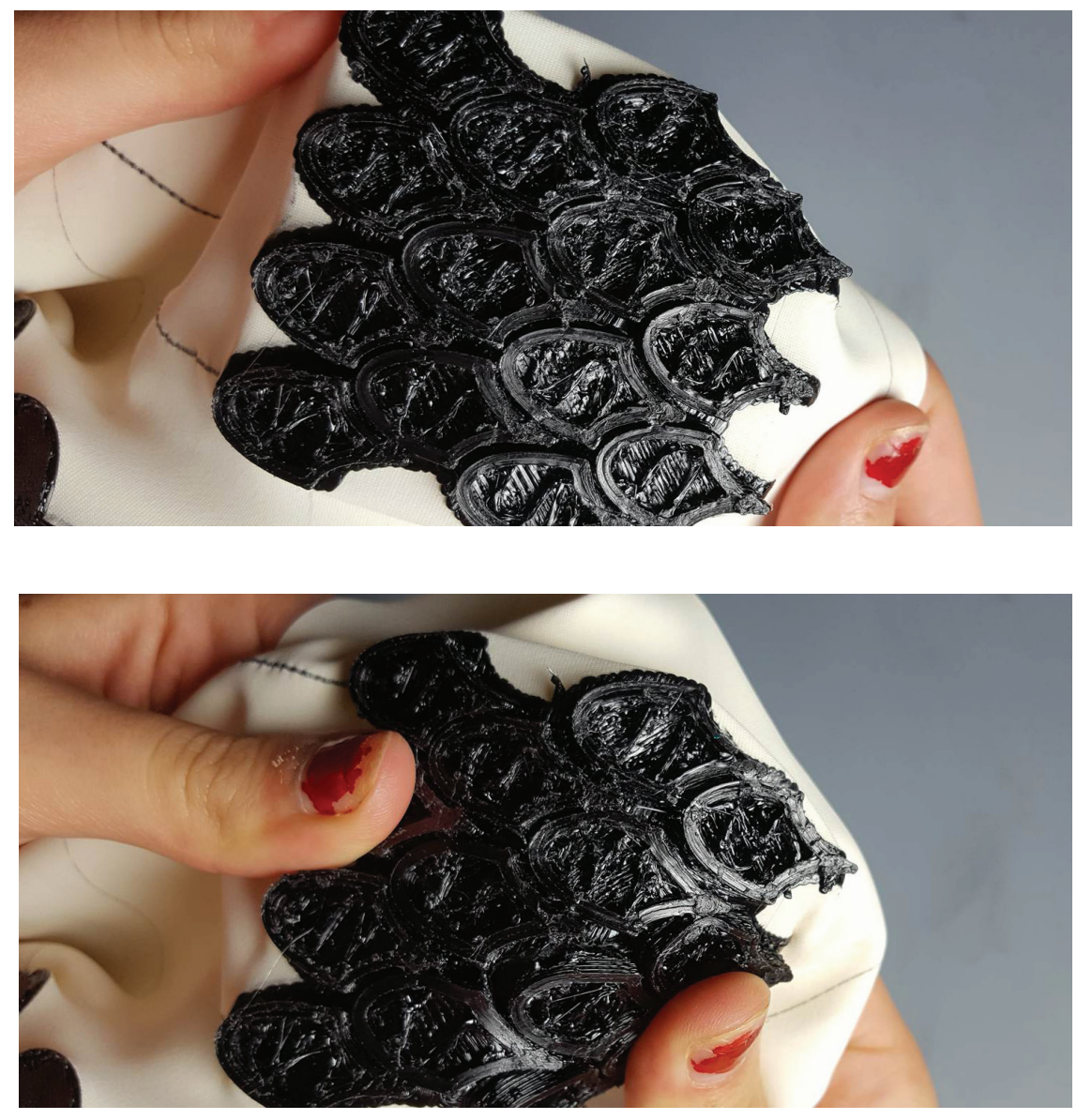

Figure 4.13

$3 \mathrm{~d}$ printed scale structure with increased height to evaluate movement restriction. 

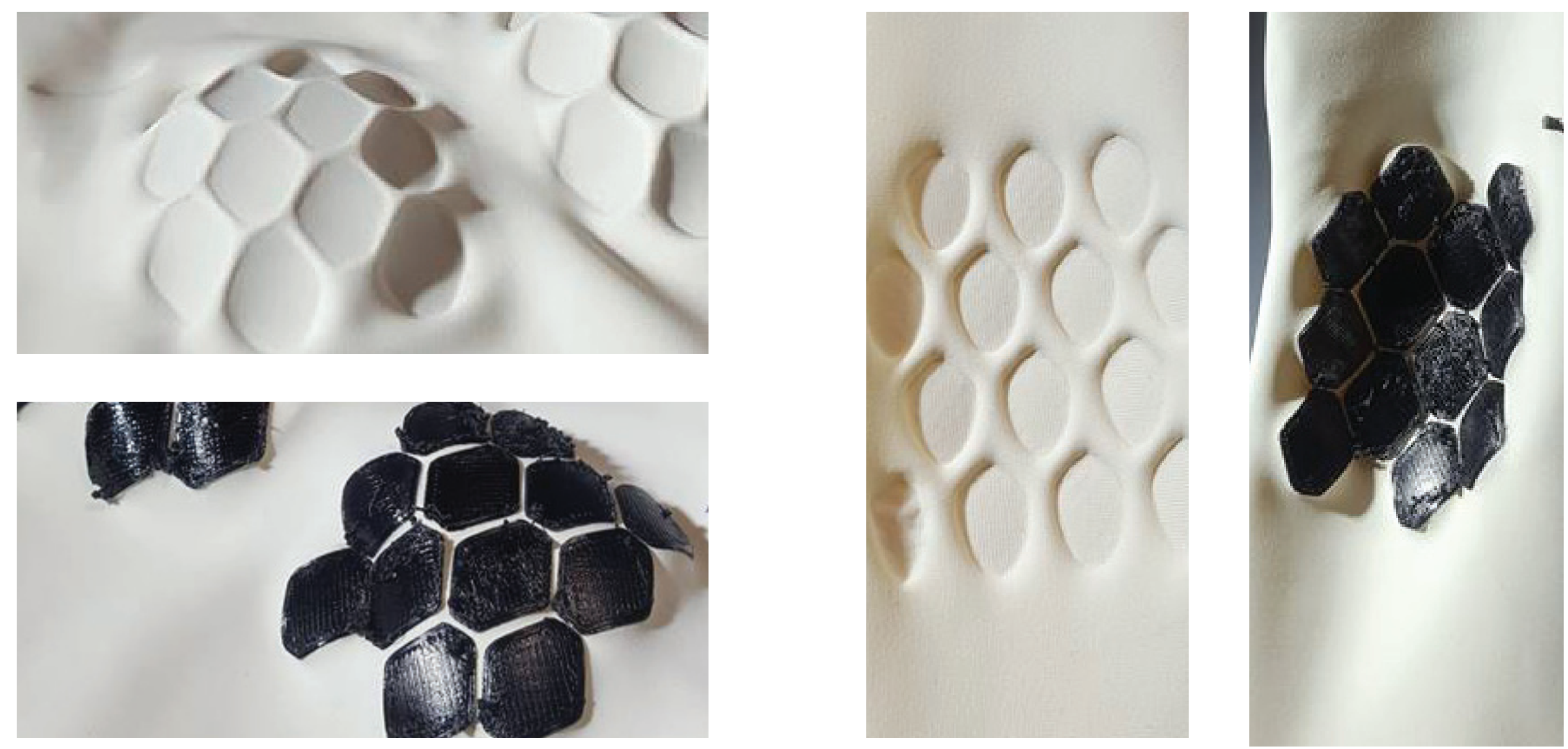

Figure 4.14

Demonstrating the behaviour of hexagon scales and its contouring capabilities. 


\section{Dynamic and Layering}
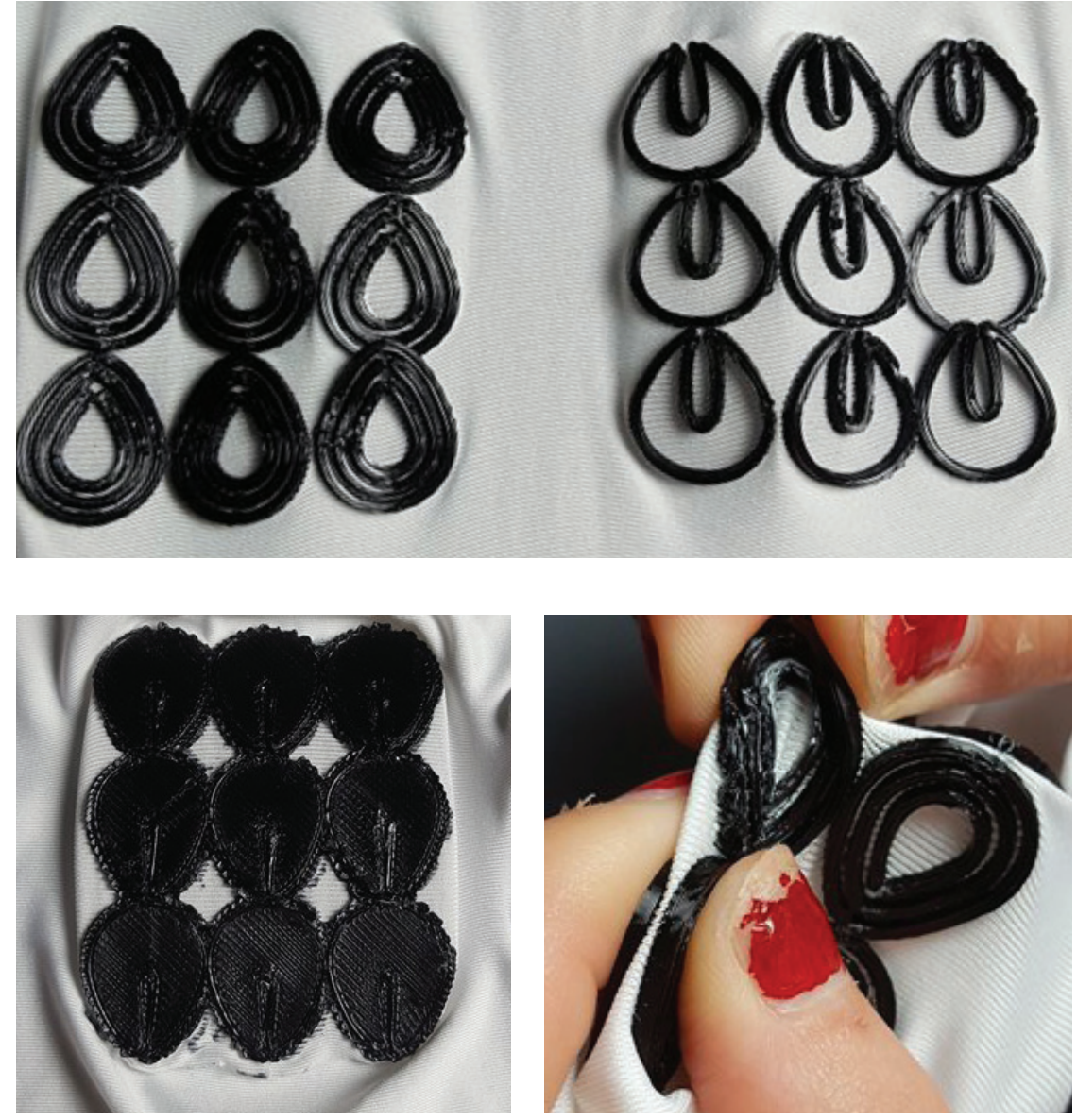

Figure 4.15

Analysing the dynamic qualities of minimal scales for additional flexibility.
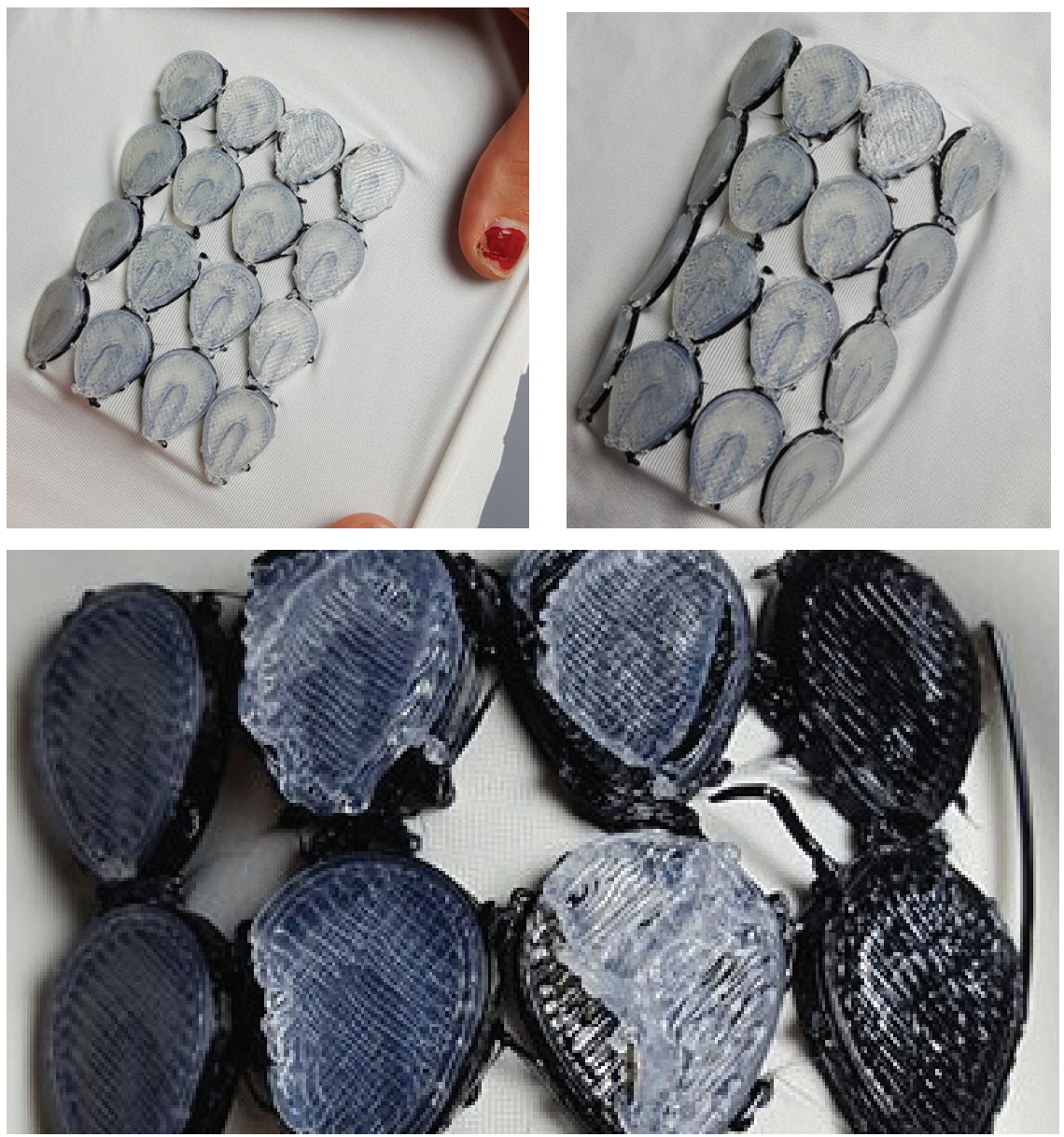

Figure 4.16

Exploring the combination of two $3 \mathrm{~d}$ printing filaments, to enhance protective capability. 


\section{Reflection}

From the first set of samples, protective qualities did become apparent through design evaluations. The locking mechanism performed effectively as well as the capability to protect the skin from abrasions. The locking system between each scale were developed further when stretching the textiles were introduced. Creating an ergonomic contour for fit ,but also part of the design functionality.

This was experimented with further to where the front edge $3 \mathrm{~d}$ prints were partially seperated from the textile releasing tension and shifting each line of scales forward. When the shift occurred, the scales were able to overlap with one another, providing extensive locking but also preserving enough dynamic. However, when the complexity of the design was advanced, other design flaws became apparent. By lifting the scales for overlapping, the bond quality between the textile and the $3 \mathrm{~d}$ print was reduced drastically. Making the scale structure too fragile to withstand repetitive impact, and based on this reason this design approach was discontinued. 3d printing with TPU(flexible), caters for a vast amount of dynamic capability which enhanced comfort as it behaved more like a second skin. But if printed too thin, the locking capability of the design was lost due to the flexibility. A solution was found by simply increasing the height of the design and decreasing the distance between each scale. Through this, the space for movement between each scale were reduced. Furthermore, by controlling the height of the printed scales the degree of flex can be controlled. Dynamic qualities of the scale designs were redesigned by altering the traditional snake scale formation to produce greater movement, reinforced by the flexibility of the TPU. This design decision was practicle as more dynamic movements were shown when simplifying the amount of material used. However, the flexibility and surface hardness were required to provide protective functionality. Samples using multiple filaments such as PLA and TPU were printed to investigate if this may be a valid way of balancing ergonomics and functionality. Creating a tough and rigid outer layer on top of the flexible TPU to determine whether this may improve protection. However, with the addition of PLA filament the flexibility of the TPU filament was reduced drastically . 


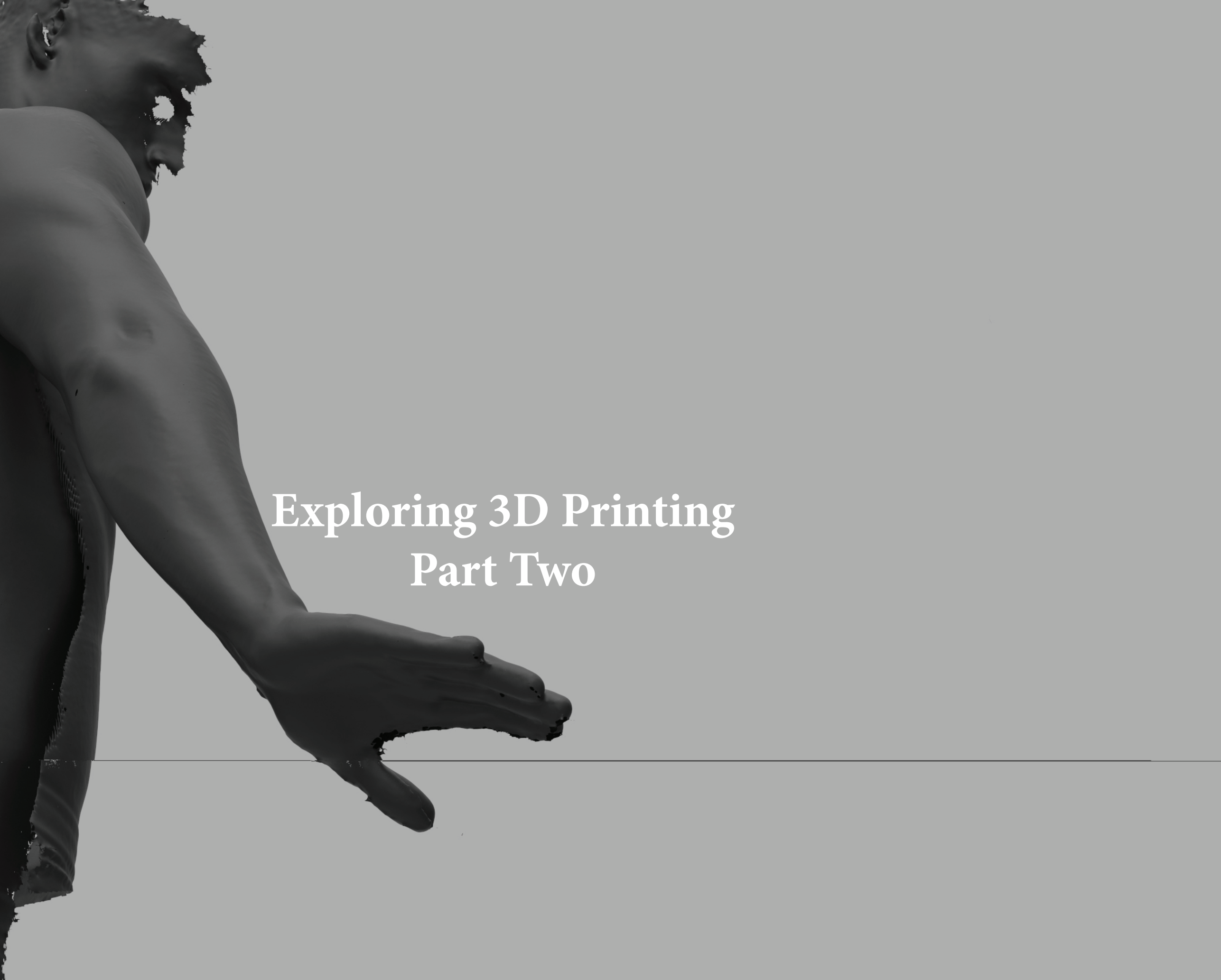





\section{Digital Scales}

Investigating how the designed scales conform to the body digitally and in physically proved difficult to resolve. Strategically placing every scale in the right position was seen as an over complicated designing process. As this would require designing scales of different shapes and sizes to not only cater for the flat surfaces of the human geometry but also those of curvilinear character. On top of this, wrapping the pattern to the geometry can also create problems, with factors such as overlapping and so on.

Instead a different approach was taken to creating a realistic, functional and ergonomic protective skin using $3 \mathrm{~d}$ scanning. When an object is scanned and imported into a $3 \mathrm{~d}$ modelling program, the mesh is made up of several polygons combined to create the $3 \mathrm{~d}$ model. From this, the idea was to utilize the digitally configured polygons and transform them into scales. Through this process, the $3 \mathrm{~d}$ generated and $3 \mathrm{~d}$ printed skin becomes customized to each individual, with every polygon and scale configurating to each unique body. Furthermore, these polygons all vary in shape and sizes, as a result of geometrical complexity. For example the elbow and shoulder are made up of several bumps and indents, therefore the amount of polygons in this area are greater and polygon sizes smaller to allow for an accurate digital model to be produced. Areas where the quantity of polygons are greater will allow for greater movement, due to polygons being smaller in turn providing greater flexibility. This method provides the skin with a geometrical network that responds to dynamic movement effectively. 


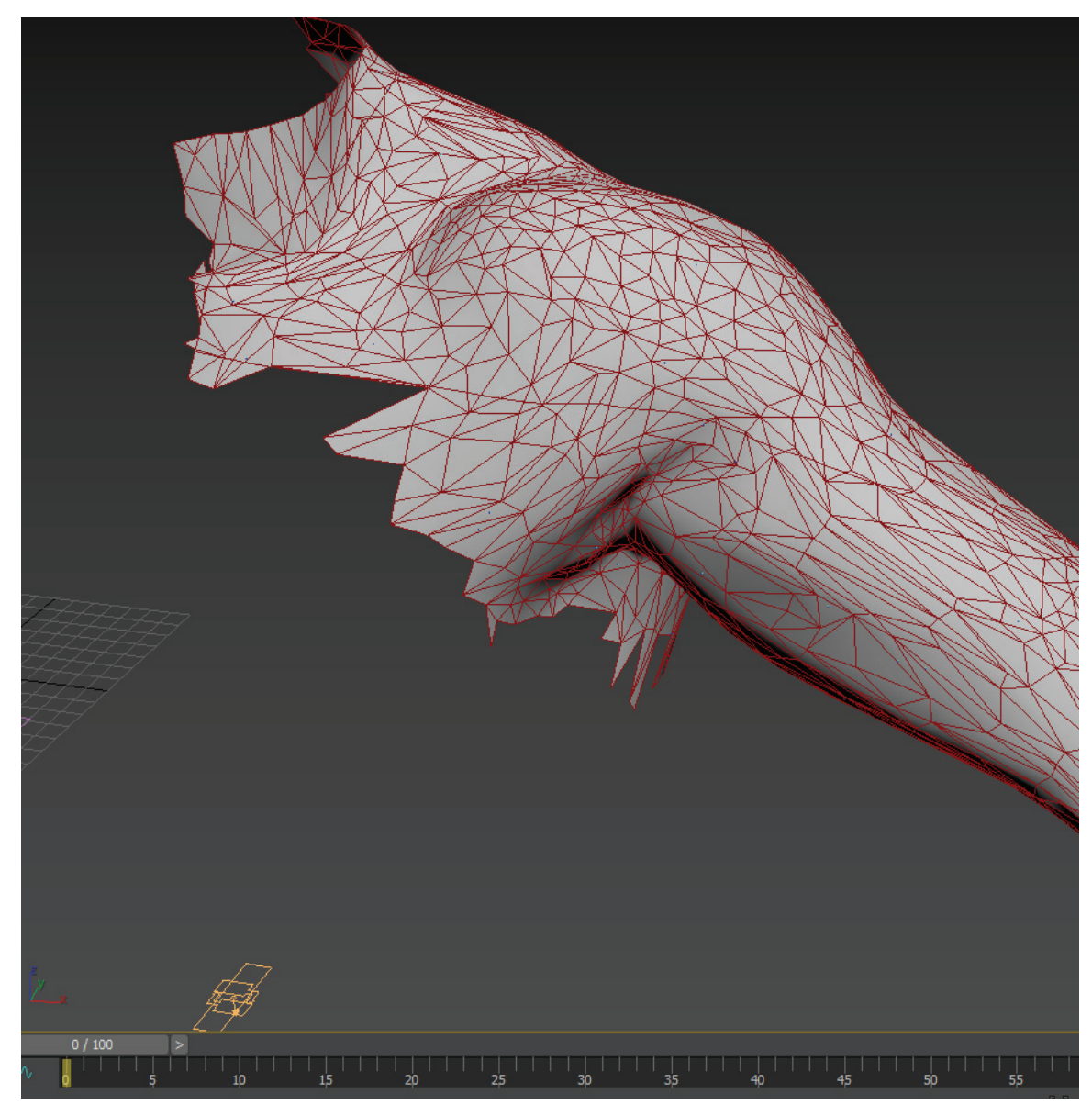

Figure 4.17

Screen capture of a $3 \mathrm{~d}$ scan, displaying the polygon configuration of the scanned arm. 


\section{Digital Scales Prints}
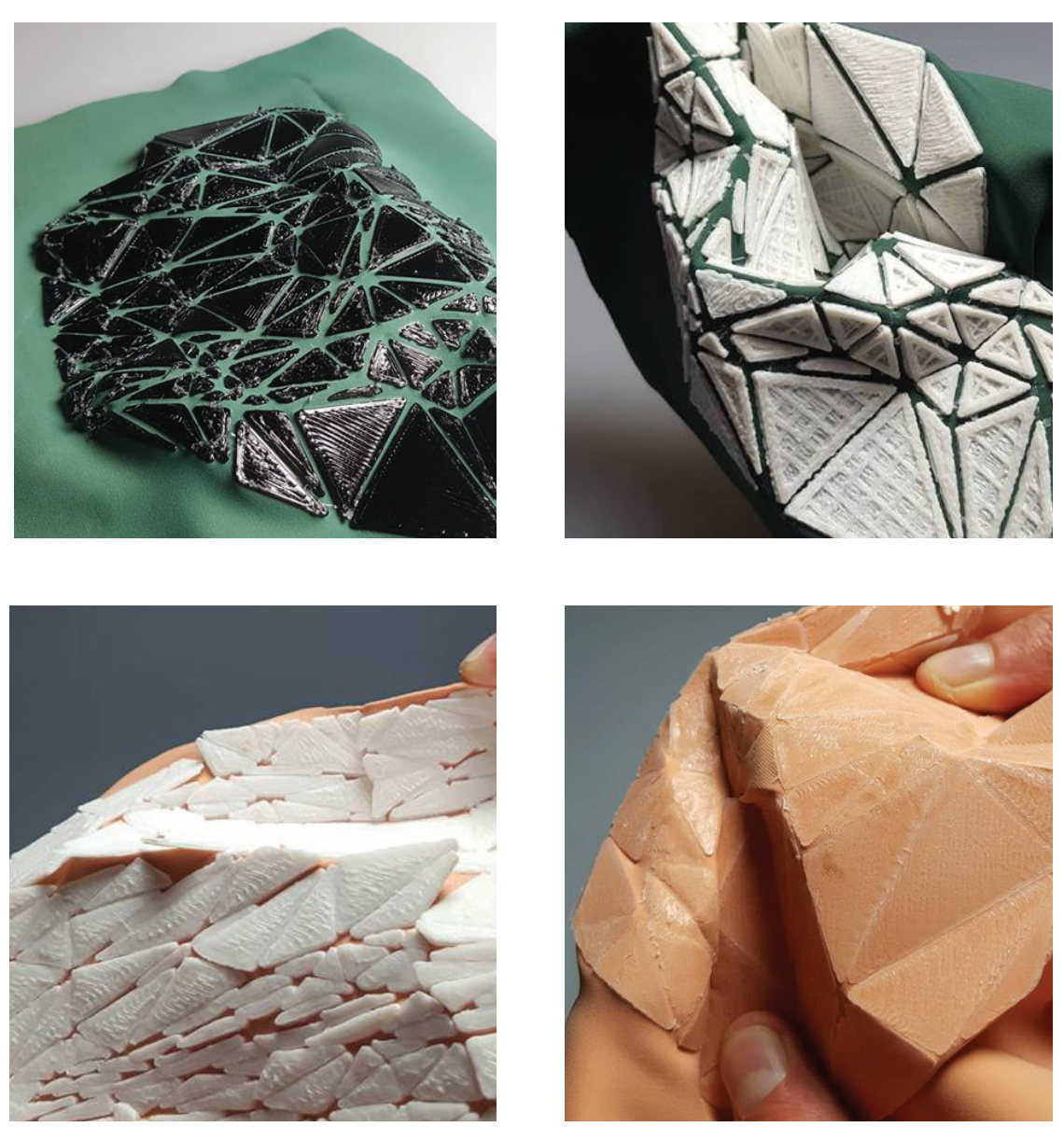

Figure 4.18

Initial $3 \mathrm{~d}$ printed polygon scales, testing different materials including ABS, TPU and PLA filament. 

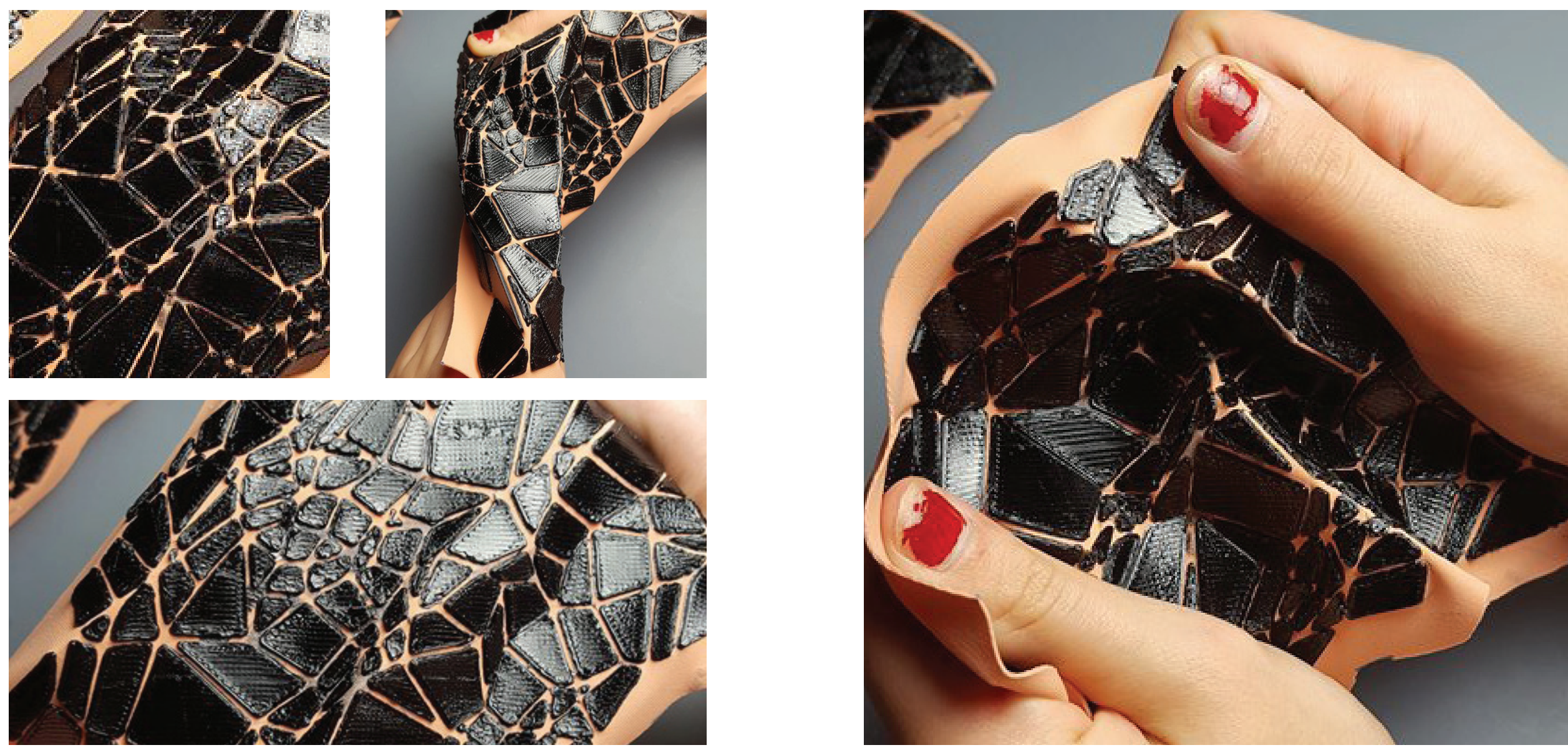

Figure 4.19

Analysing the generated polygon scales in terms of dynamic aesthetic qualities, post $3 \mathrm{~d}$ print. 

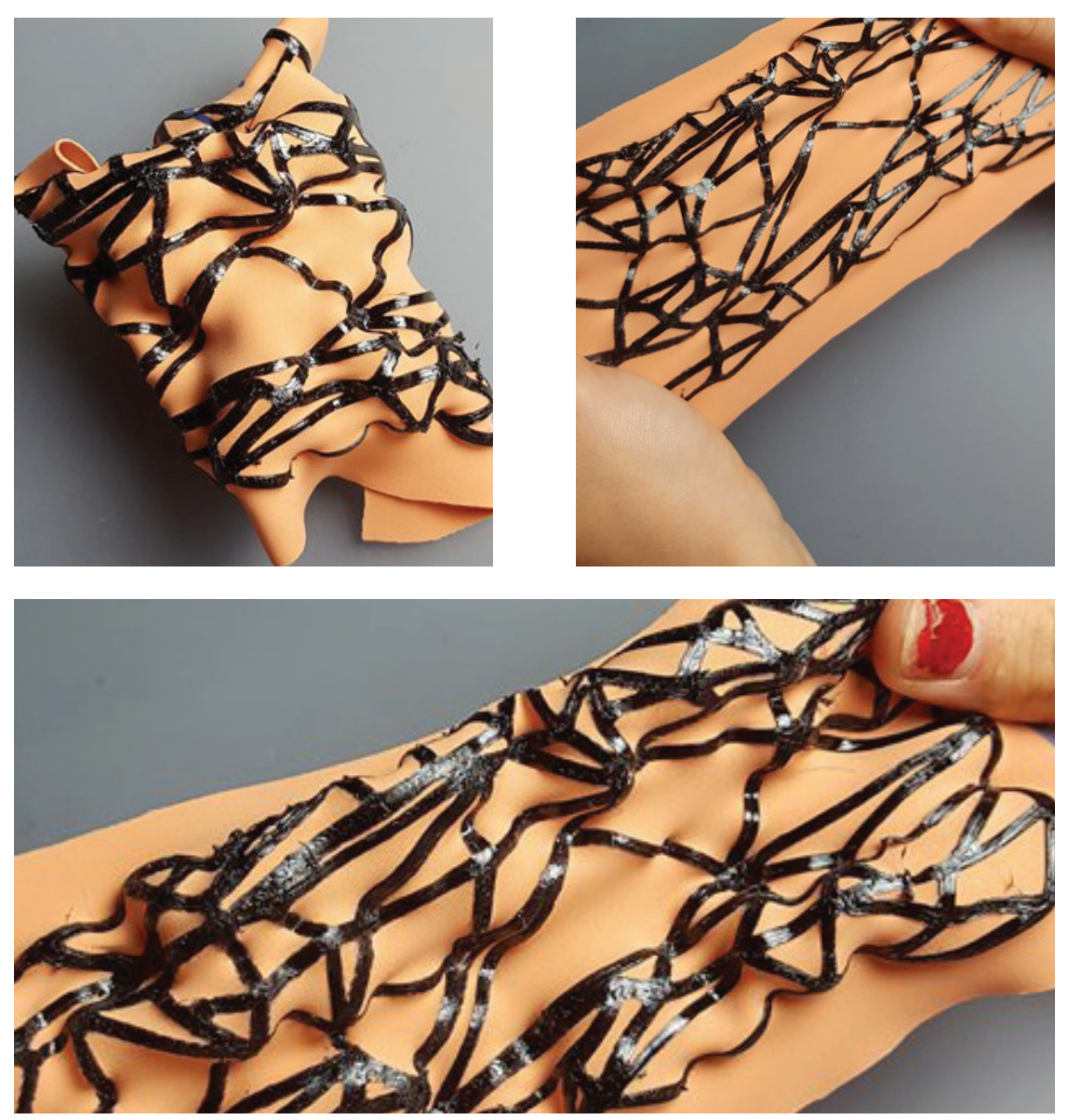

Figure 4.20

Test sample investigating the edge lines of digital scales. Inspecting how this same behaves in comparison to thedigital scales.
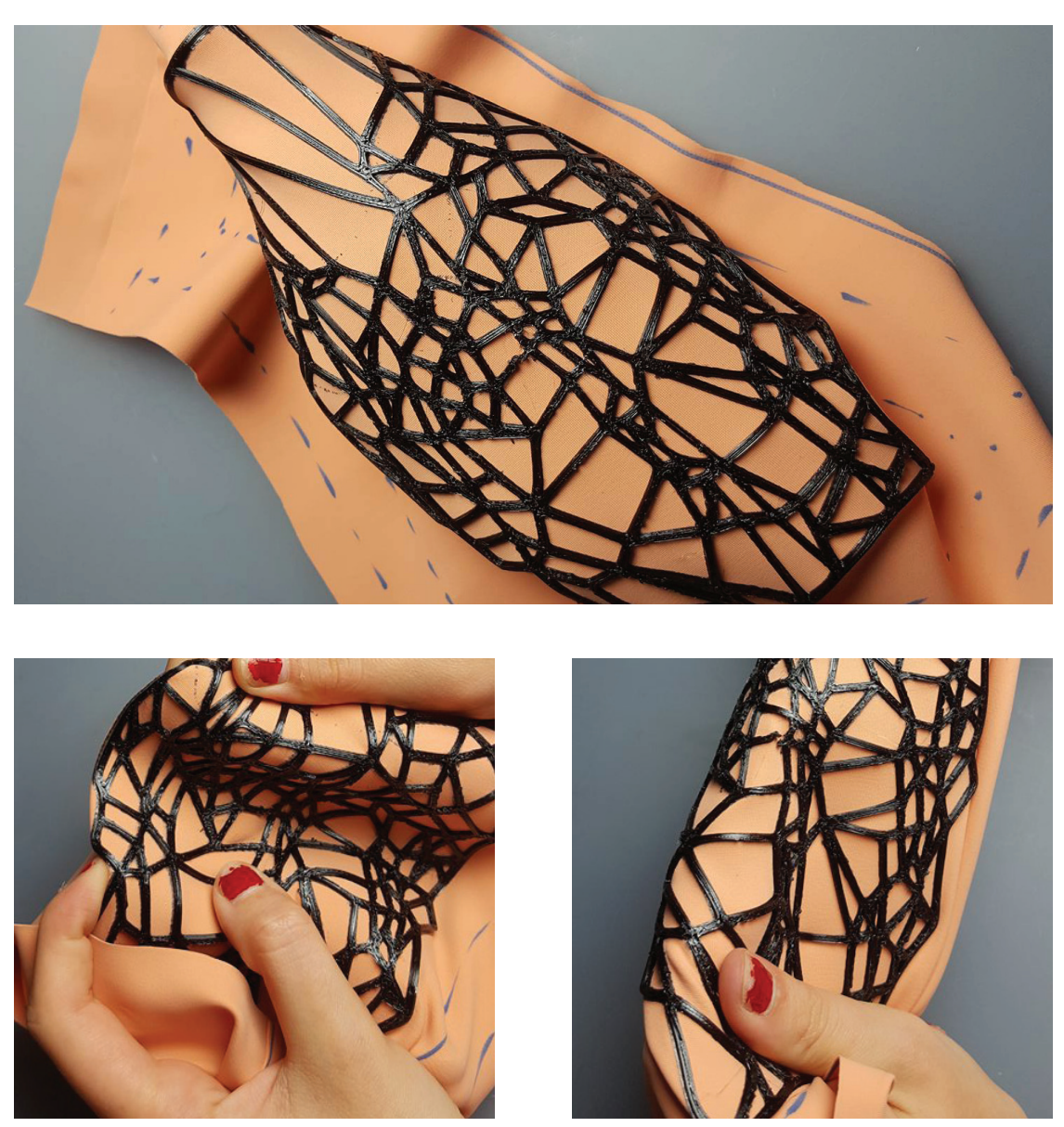

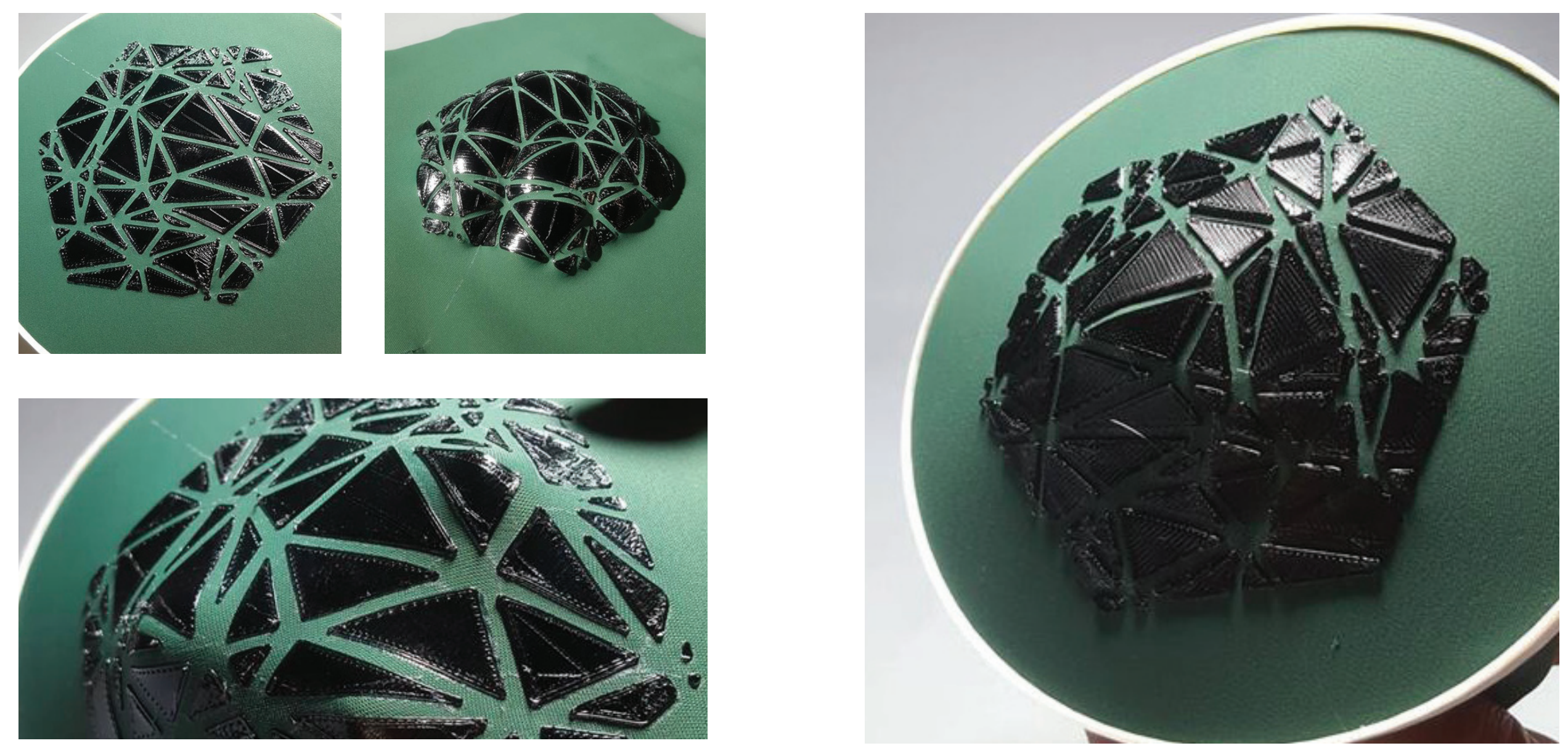

Figure 4.21

Demonstrating the scale configuration when applied to an organic surface, and or stretched around a surface. 

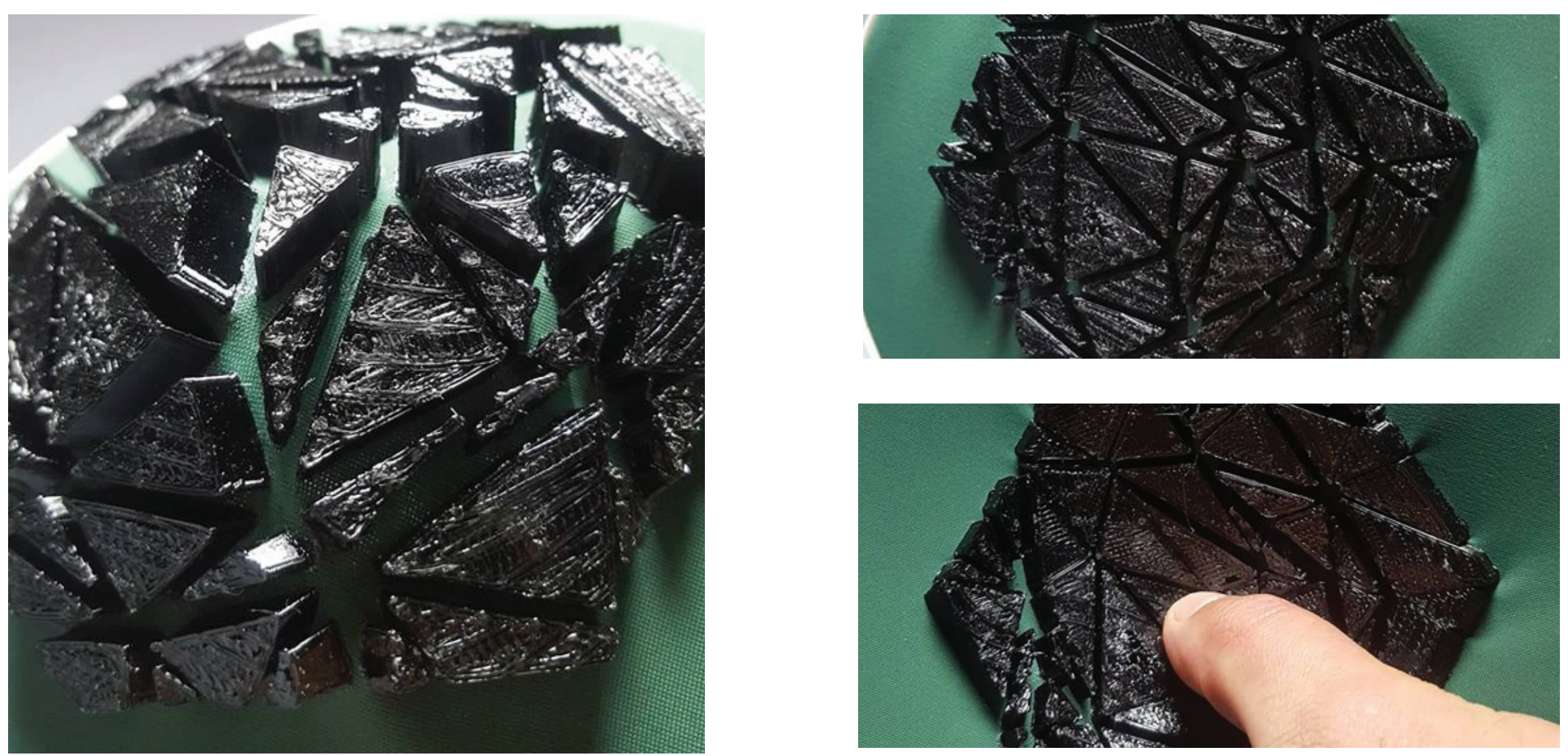

Figure 4.22

Showcasing the locking and expansion of scales when an external force is applied. 

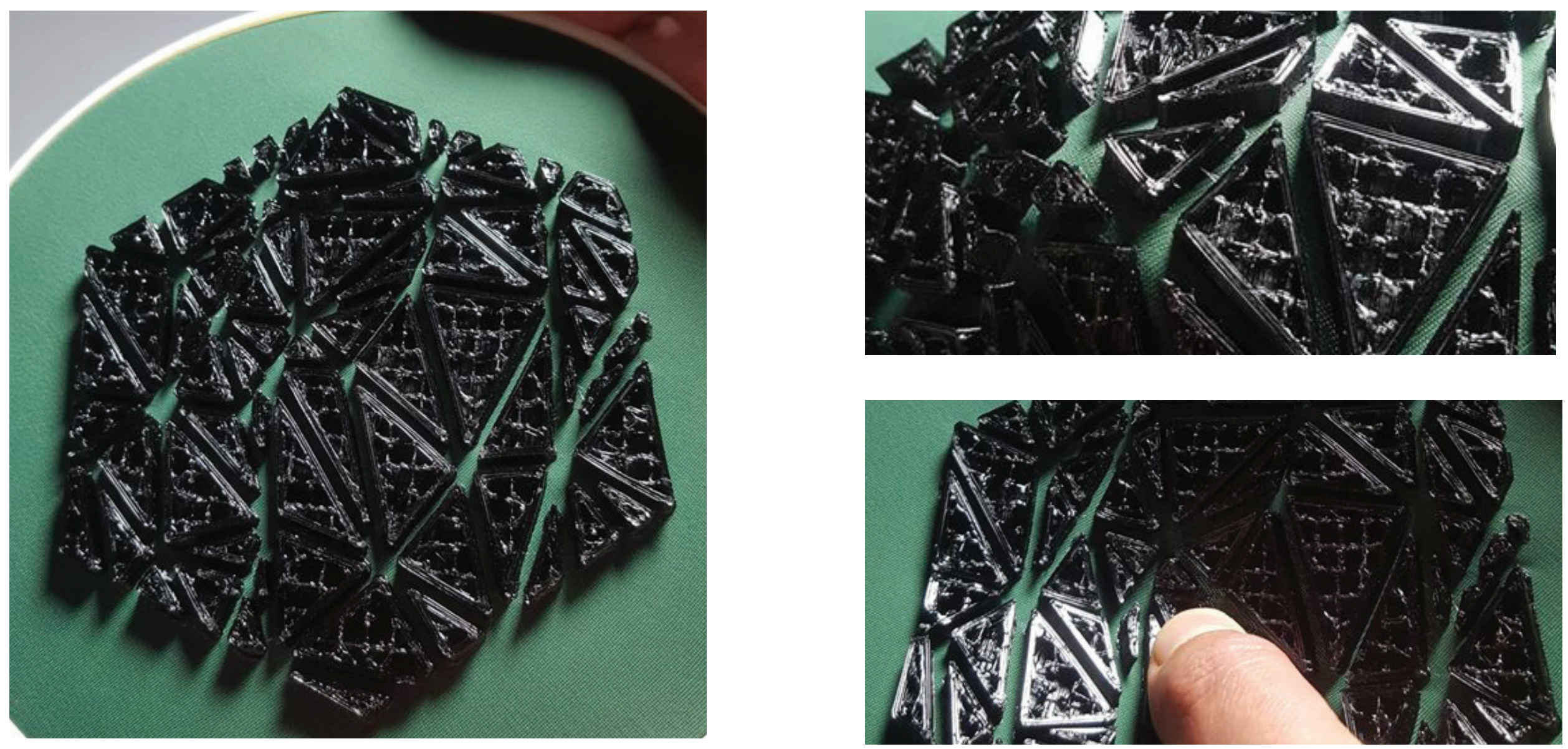

Figure 4.23

Sample print was stopped to expose the internal structure of the digital scales. 

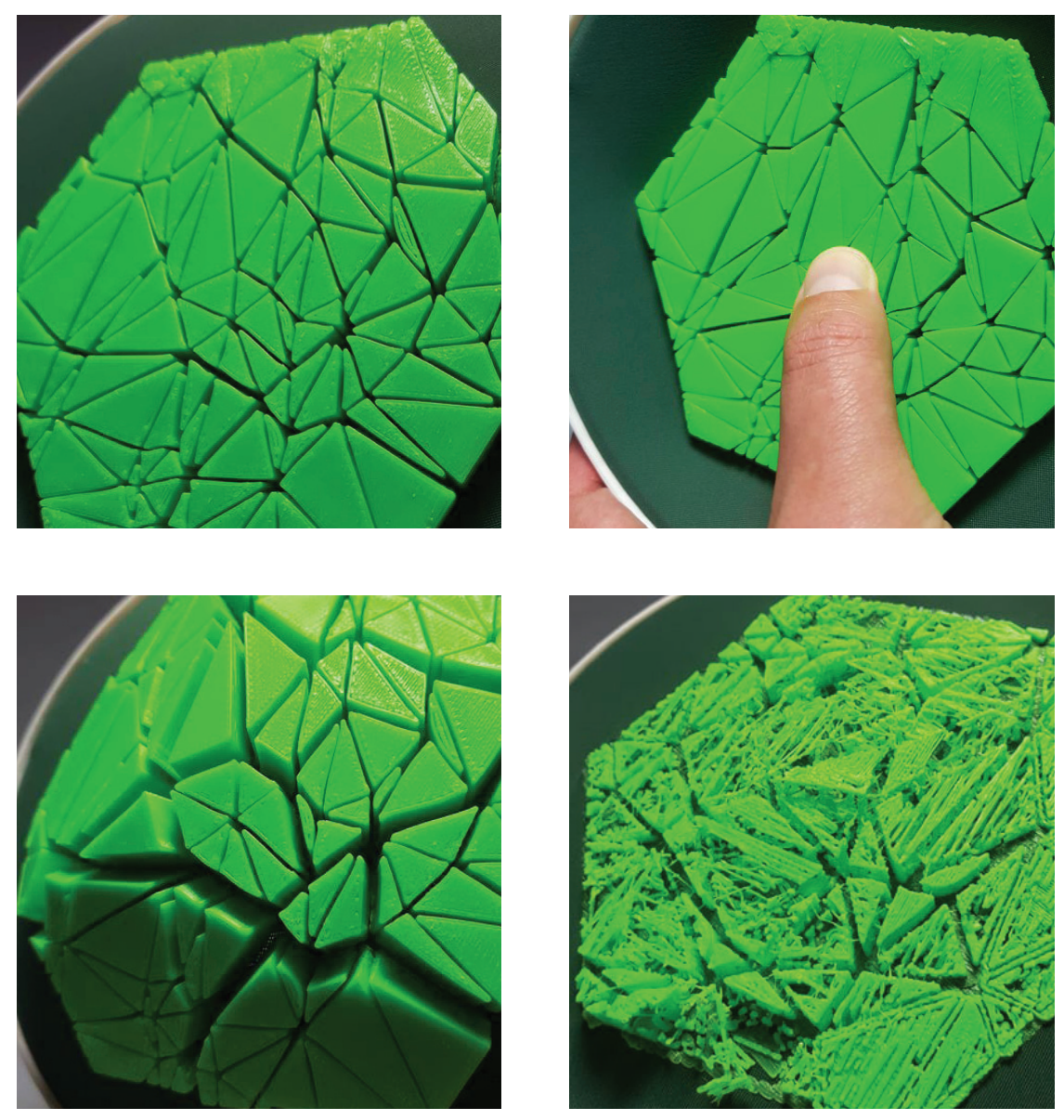

Figure 4.24

Rigid test sample showing how movement can be restricted further, through material selection and minimized space between scales. 


\section{Reflection}

The same dynamic restriction strategy was applied to the digital scales, where by the height and distance between each scale was decreased or increased depending on the dynamic objective. In this case, minimal $3 \mathrm{~d}$ printing height provided little to no movement restriction. While increased height can introduce dynamic limitations. Furthermore, scales with minimal height could be applicable for protection from minor cuts, scrapes and abrasions. Prints with greater heights can be utilized for impact protection as well as joint stability through using the locking mechanism. The aesthetic of the thinly $3 \mathrm{~d}$ printed scales were perceived to be more skin- like due to its subtlety. While higher prints provided potential for impact absorbing opportunities. Printing the digital scale edges showed distinct differences in terms of tension management. Thinly printed lines demonstrated greater conformal capability but less controlled. Whereas edge lines with greater height showed less conformal ability yet more control. This provided some insight as to what factors to consider when creating the root/ vein system, although a physical model will not be produced in this thesis. 


\section{Growing Support}

Increasing the thickness of the scales provided a way of creating design restrictions, this also began the idea generation for how impact absorption can be introduced. From this, impact protection became possible and a design phase. Thicker print samples were produced, to investigate the impact absorption qualities of the TPU filament. Initial tests included raising the height of scales, with no in-depth consideration the underlying structural design. This method did not prove successful as the material did not maintain the same flexibility and softness after continuous layering. Therefore, through design evaluations this would not be a comfortable nor functional design approach. To add complexity and provide innovative functionality, greater design thinking was required, considering the given tools and its capabilities.

Test models were designed to spark ideas, looking at how the materials available and several design approaches can be combined to create a design which has impact absorbing qualities. A design which used simple vertical miniature pillar structures were tested and were somewhat successful but had no complexity. Furthermore, suspension was created and proved to be somewhat succesfull. However, when tested at a smaller scale, failed due to the $3 \mathrm{~d}$ printer not having a high enough resolution to carry out a highdetailed print. This design also proved to be too expensive and had a risk for error too high to be invest in.
From this, understanding that to design a fully tested, engineered protective system is not the end goal of this thesis, renforced design creativity and thinking. By reassessing the tools ( $3 \mathrm{~d}$ printer) a new approach to designing a protective structure was developed. Through exploiting he $3 \mathrm{~d}$ printing software an idea to grow a protective structure became possible. As mentioned before, raft and support material are ways the $3 \mathrm{~d}$ printer ensures models are steady and supported during the printing process. From this, an idea surfaced where if one were to design a model which encourages or tricks the $3 \mathrm{~d}$ printer into printing its own strategically placed structures, this could be a way of validating a structure able to absorb impact. Through this design approach, the design work load was reduced drastically. To carry out this design method, the design approach was changed, whereby raft and support material became part of the design. Designing models which have no base, instead floats in midair or has drastic over hangs were created to force the $3 \mathrm{~d}$ printing software to build its own supportive structures and support the designed model. 

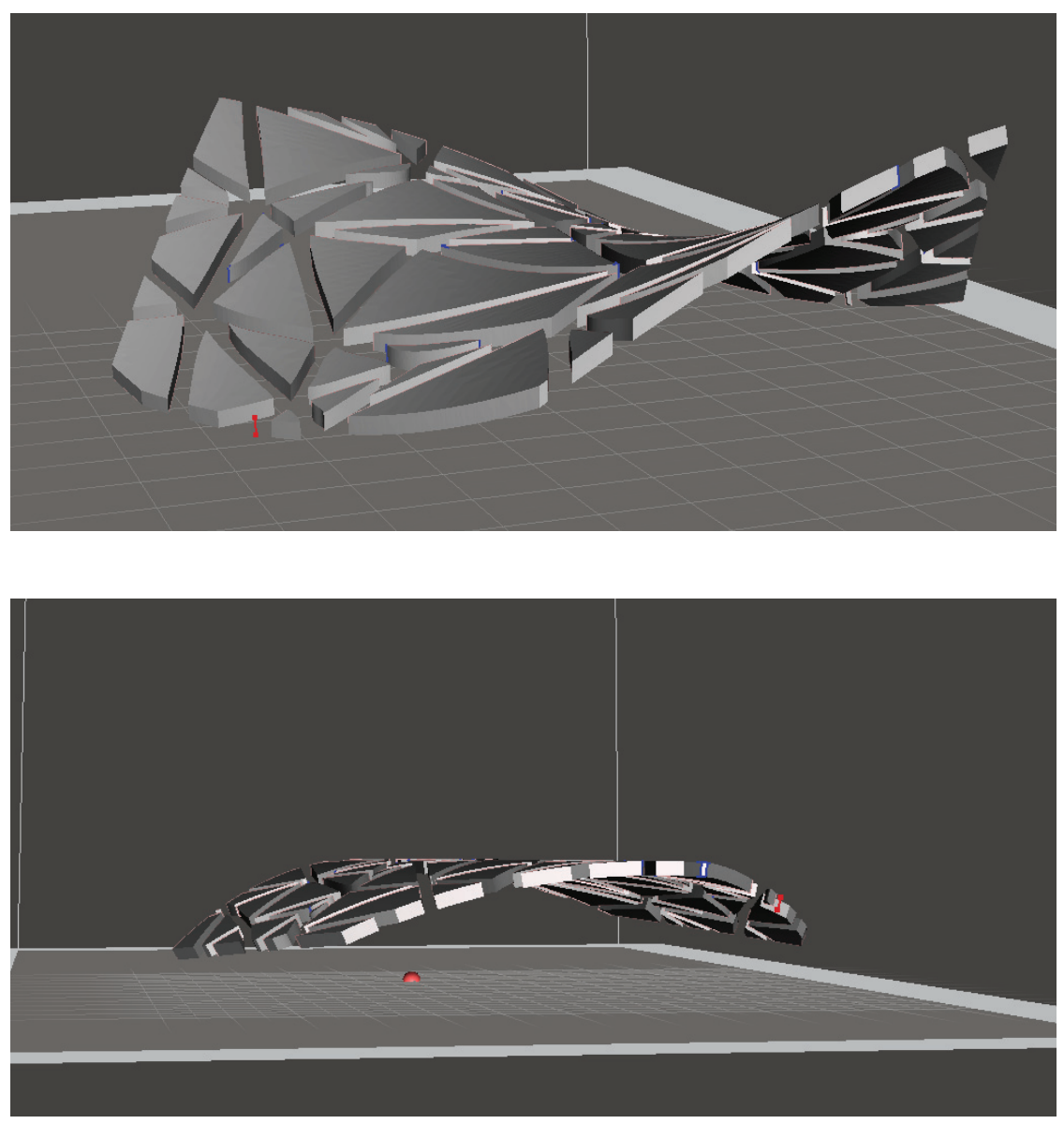

Figure 4.25

Floating $3 \mathrm{~d}$ models to allow support material to be generated and built by the $3 \mathrm{~d}$ printing software (UP Studio) 

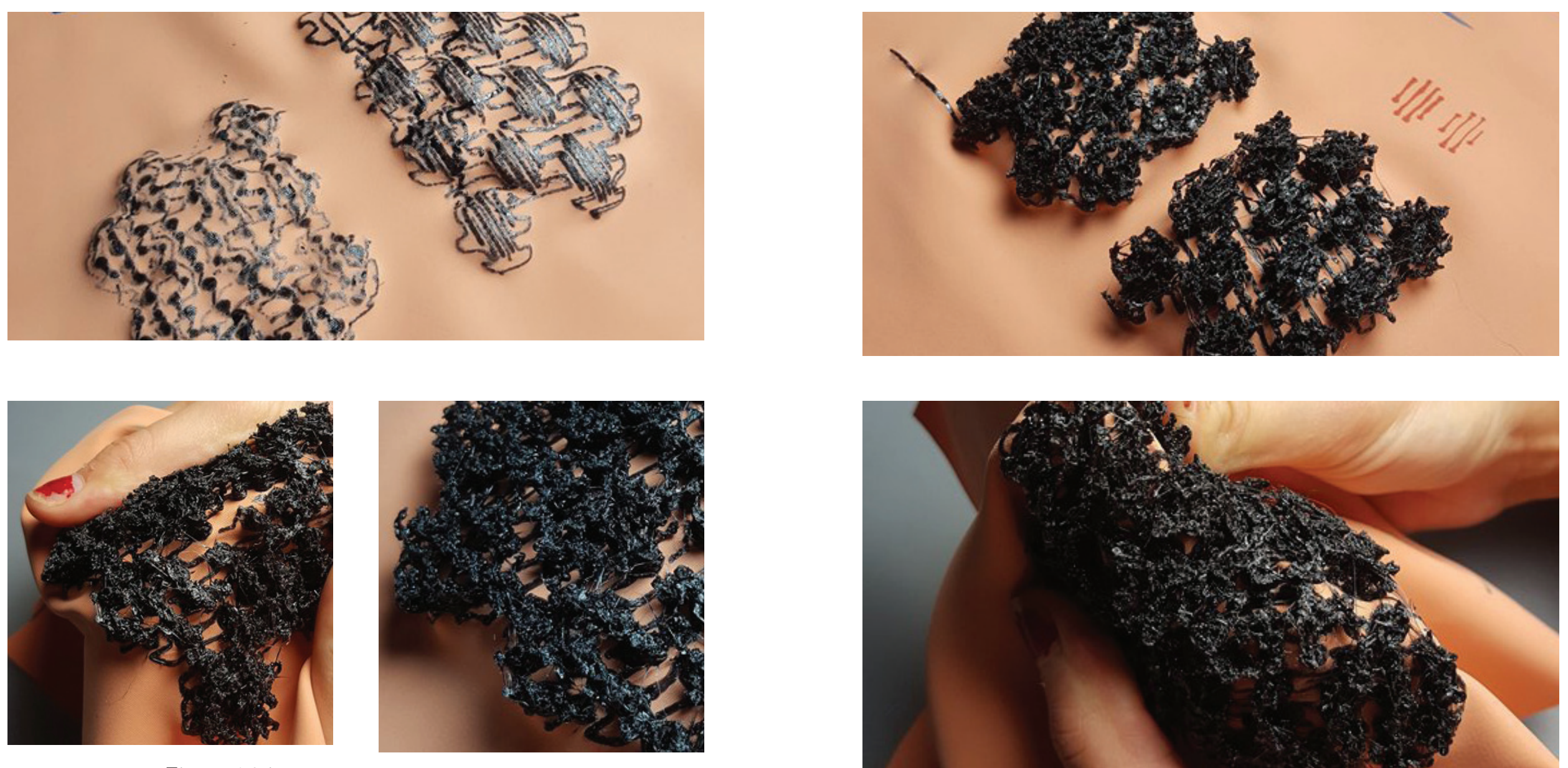

Figure 4.26

Growing an impact absorbing mesh using $3 \mathrm{~d}$ modelling and the $3 \mathrm{~d}$ printing software. 

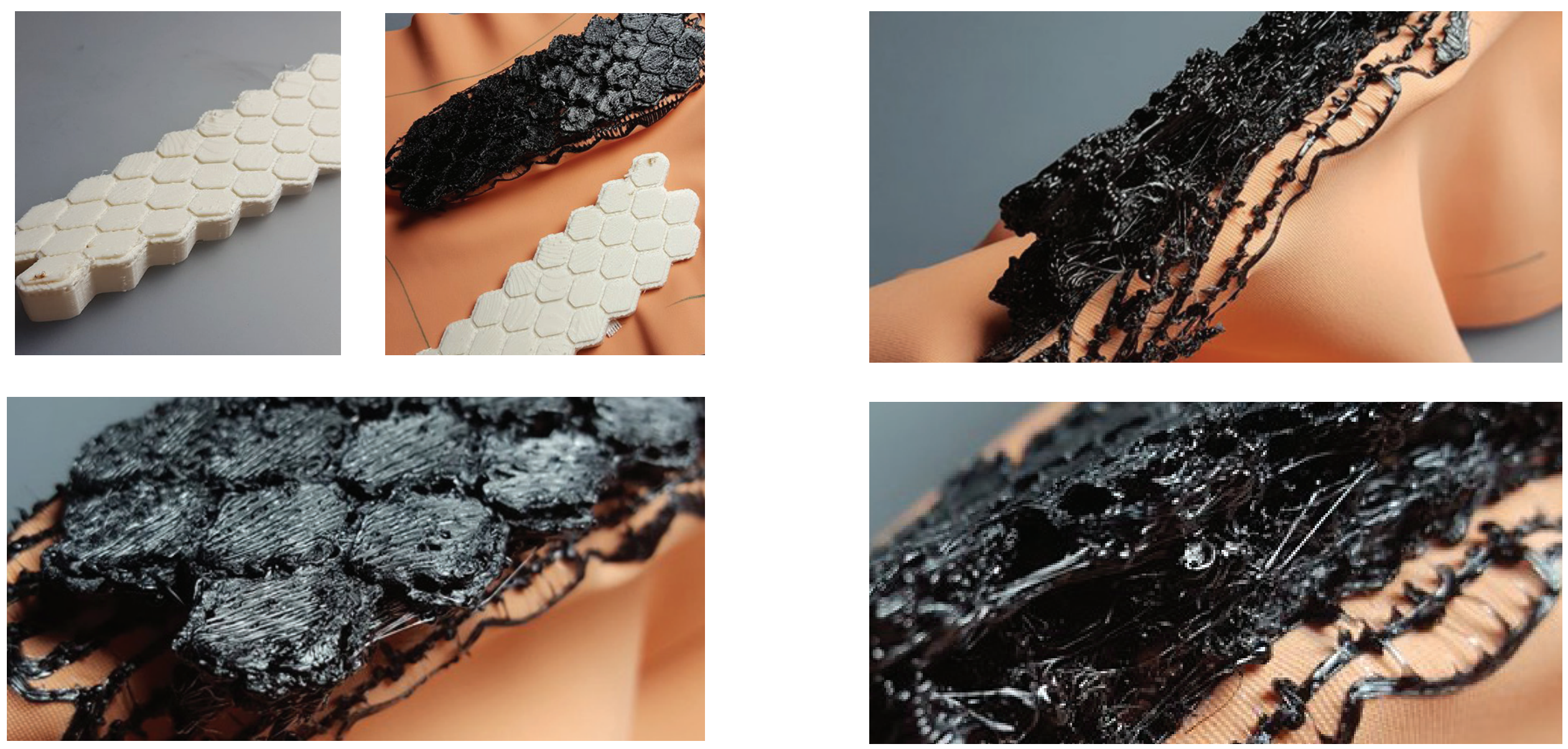

Figure 4.27

Evaluating the TPU raft and its impact absorbing legitimacy based one design reflection. 

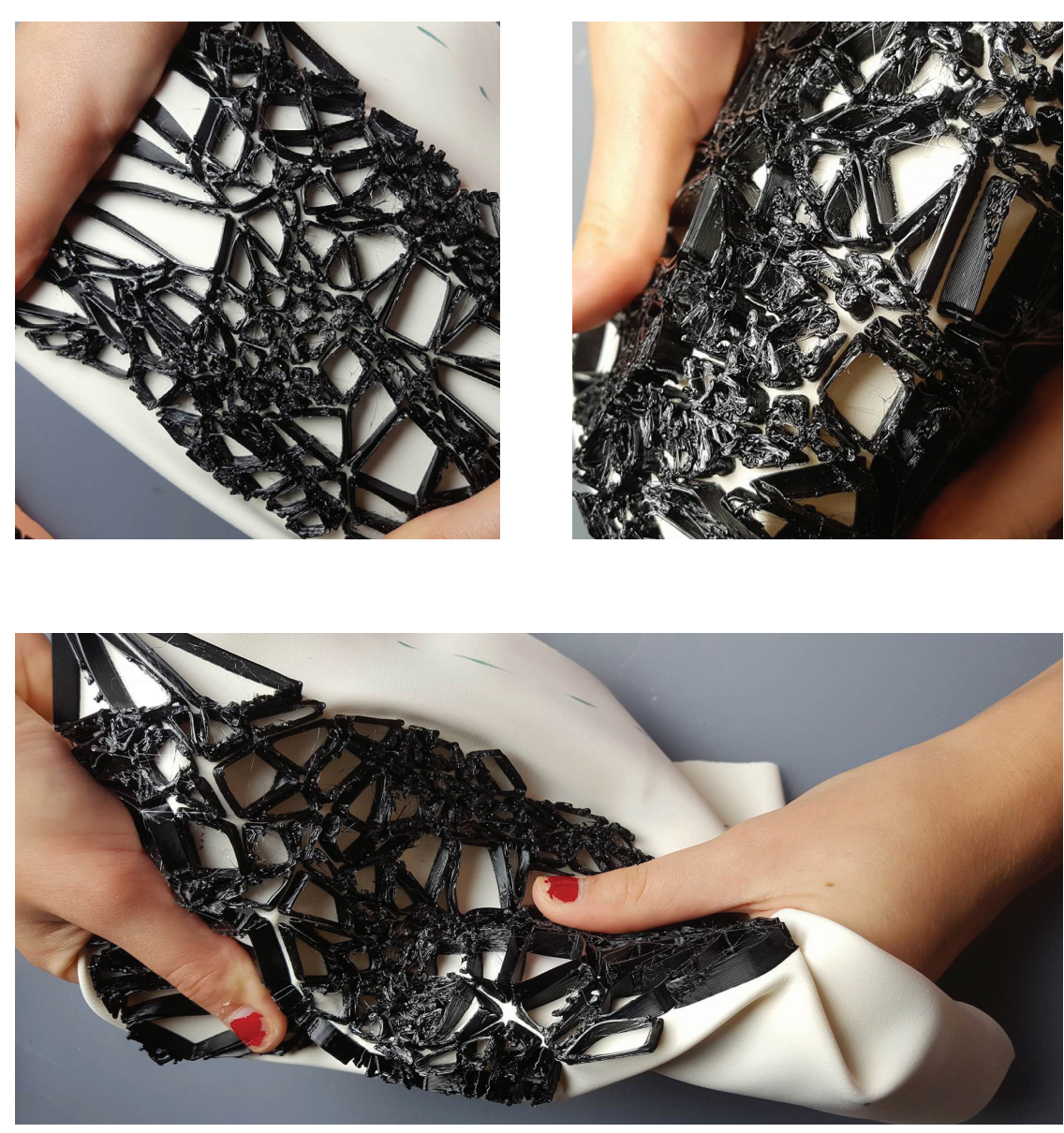

Figure 4.28

Testing the dynamic and functionality of the extruded polygon edges as a $3 \mathrm{~d}$ printed sample
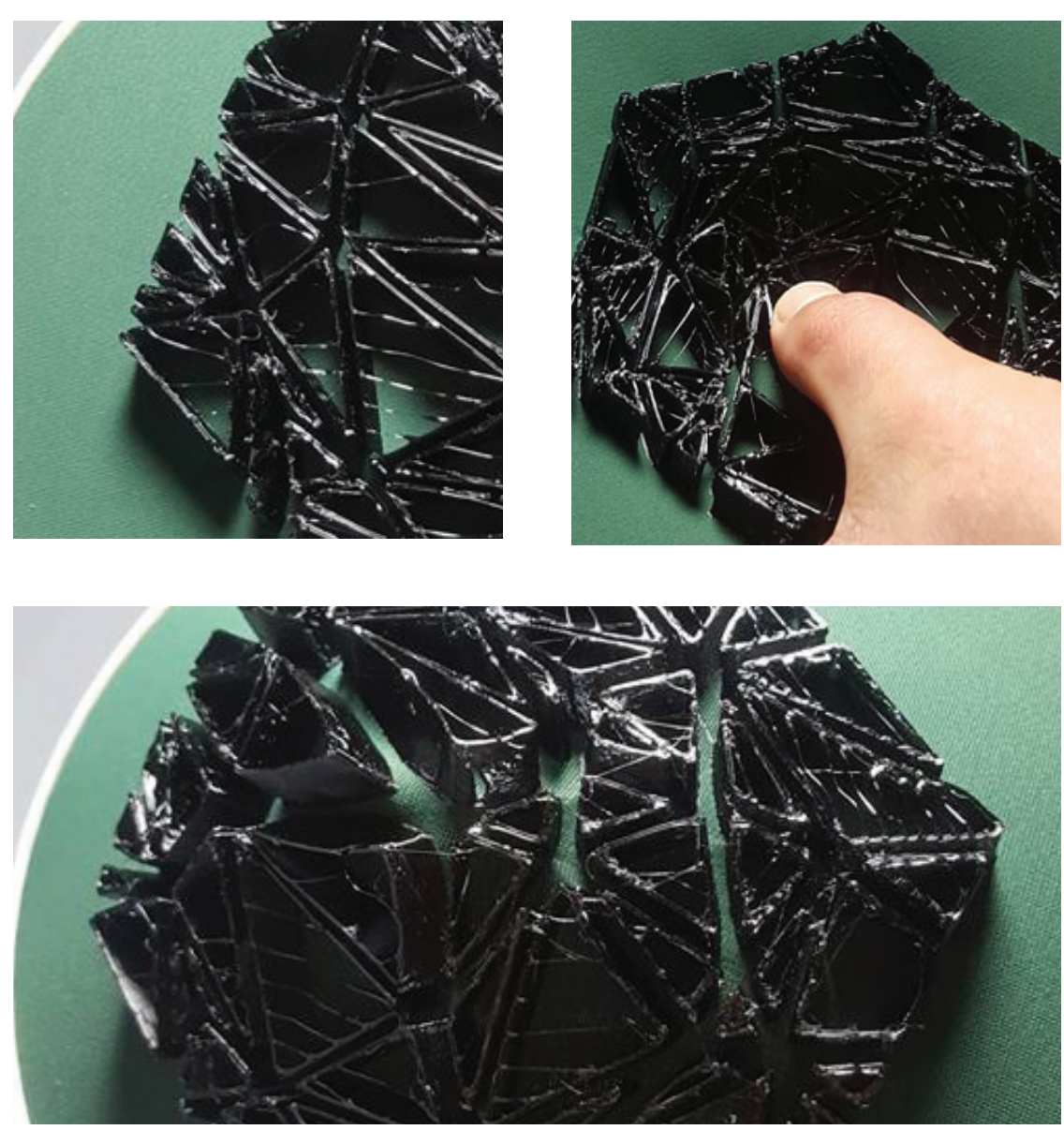

Figure 4.29

Evaluating the validity behind using $3 \mathrm{~d}$ printed support material as a strategy to respond to impact 

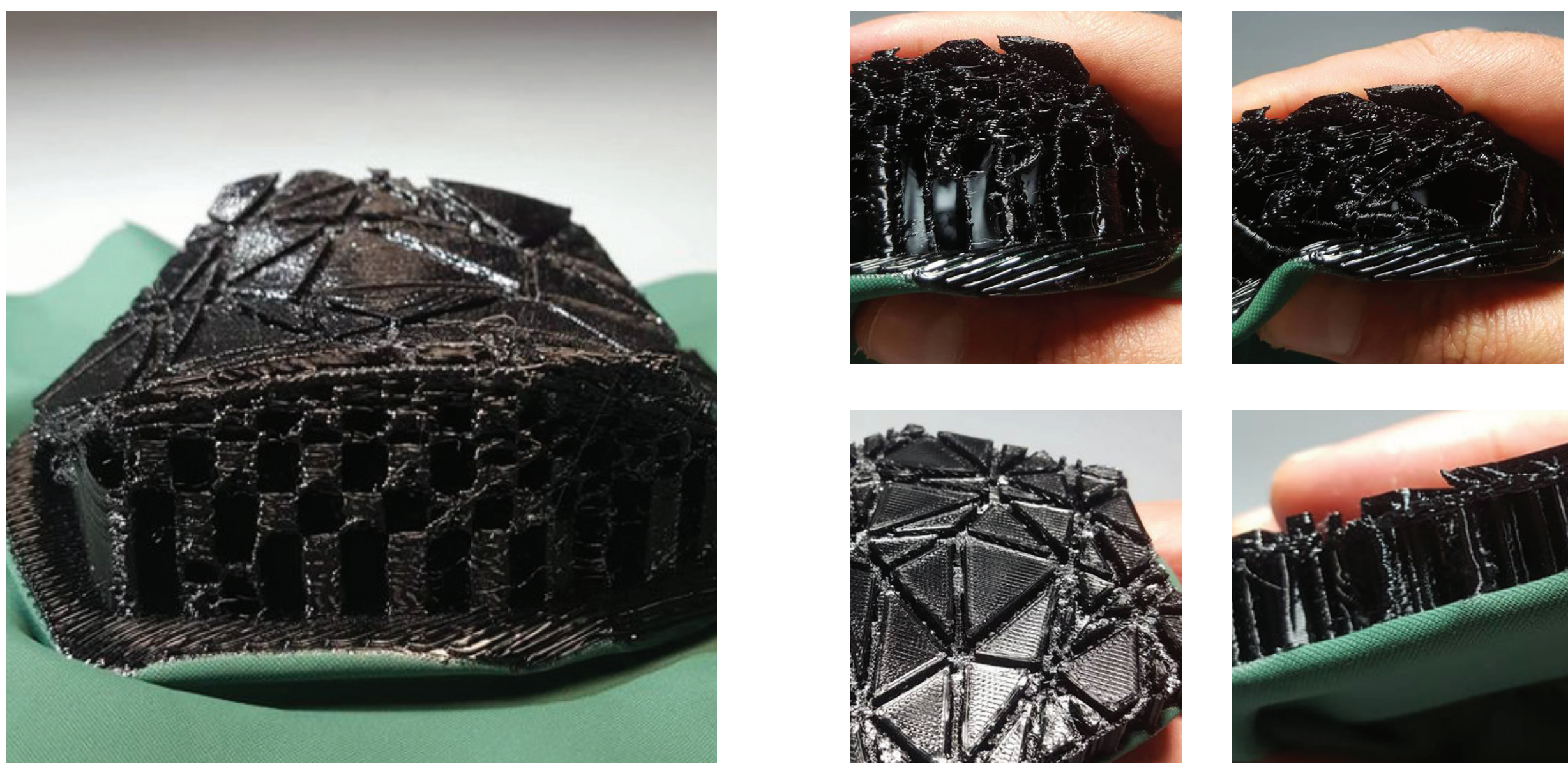

Figure 4.30

Analysing the functionality of the support through feeling and research through design.
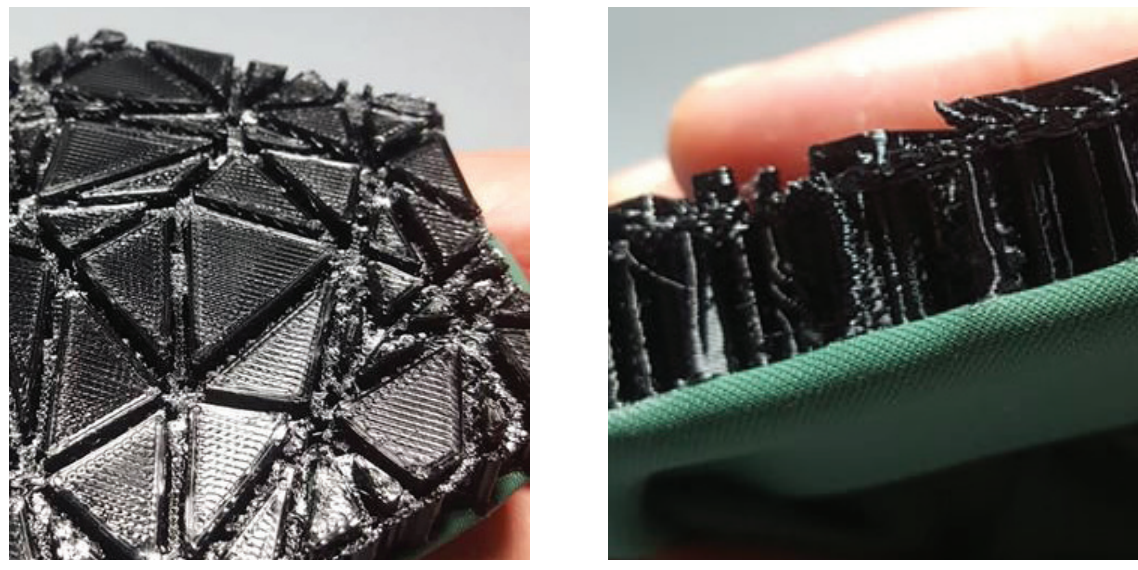

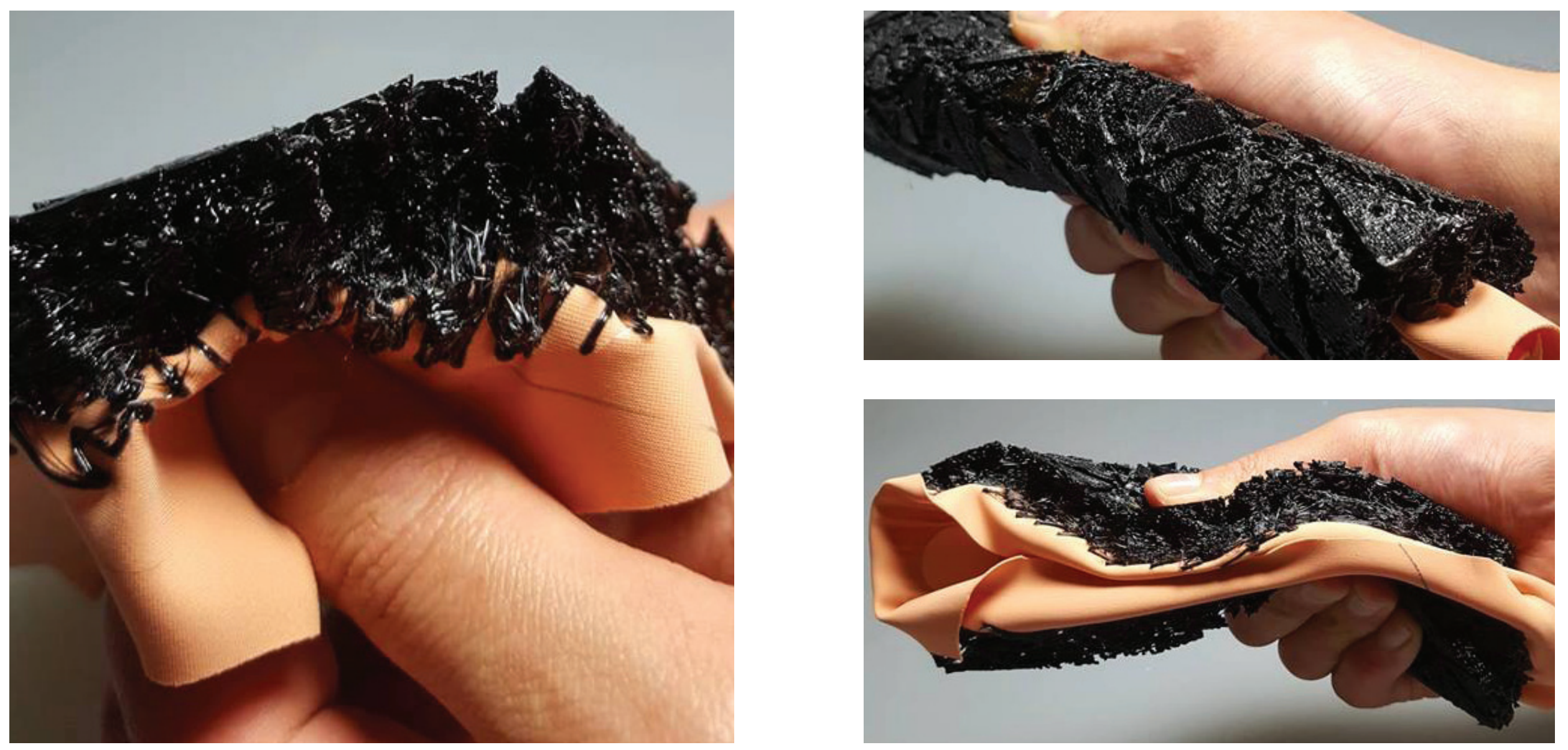

Figure 4.31

Analysing the functionality of the support through feeling and research through design. 


\section{Reflection}

Samples were created and carried out successfully, models showed great flexibility and impact absorbing qualities based on touch. As the supportive layers are quite thin the models were able to be squished due to the thickness of the support structure as well as the flexibility of the $3 \mathrm{~d}$ printed material. To stiffen or soften the mesh or structure, supporting structures were added and removed during the $3 \mathrm{~d}$ modelling process. Providing the samples with variation and further design evaluation. However, in comparison with the earlier digital scale samples where each digital scale or polygon is separated by a few millimetres this does not occur in this case. As part of building the support for the floating model, the $3 \mathrm{~d}$ printer creates two raft layers one that is situated on the printing bed and another at the top of the $3 \mathrm{~d}$ printed support structure. The second raft layer at the top, is done to create a flat surface to replicate the flat $3 \mathrm{~d}$ printing bed, because of this the individual scales are unable to be separated in comparison to previous models. However, due to the minimal raft thickness and the flexible TPU filament, the model is still capable of dynamic movement. 


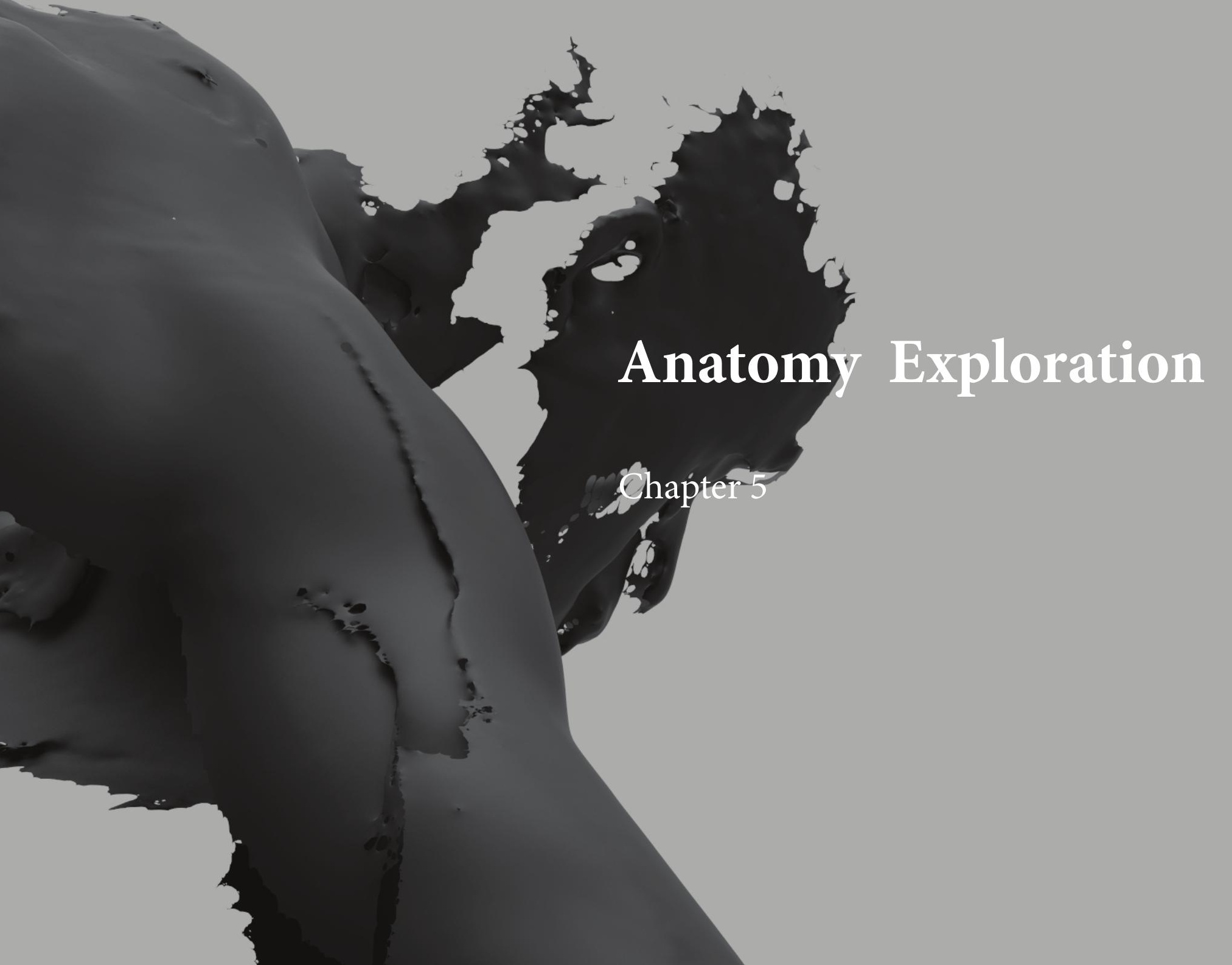


This design phase analyses human dynamic, how the body moves and how it reacts in order protect itself from impact. Exploring how the target body parts behave in and out of the design context, from this analysis can be done to determine how the protective skin needs to be designed to respond effectively. Through this rational design decisions are able to be carried out, defining how $3 \mathrm{~d}$ printing can assist the athlete and from this refine the design outcome. 


\section{Testing the Limits}

Photography was utilized to evaluate the dynamic diversity of the arm and its limitations. A design study was carried out to examine how much the arm can flex, extend, rotate etc. From this, greater understanding of the arm and shoulder joint movement can be acquired. Furthermore, design decisions can be applied dependent on this, through determining how the $3 \mathrm{~d}$ printed skin should respond to enhance functionality. Where to provide and remove flexibility will become apparent as well as how much is required. Using a gridded sleeve will highlight movement, defining how much the textiles should stretch, through analysing how the gird warps depending on the joint movement. 

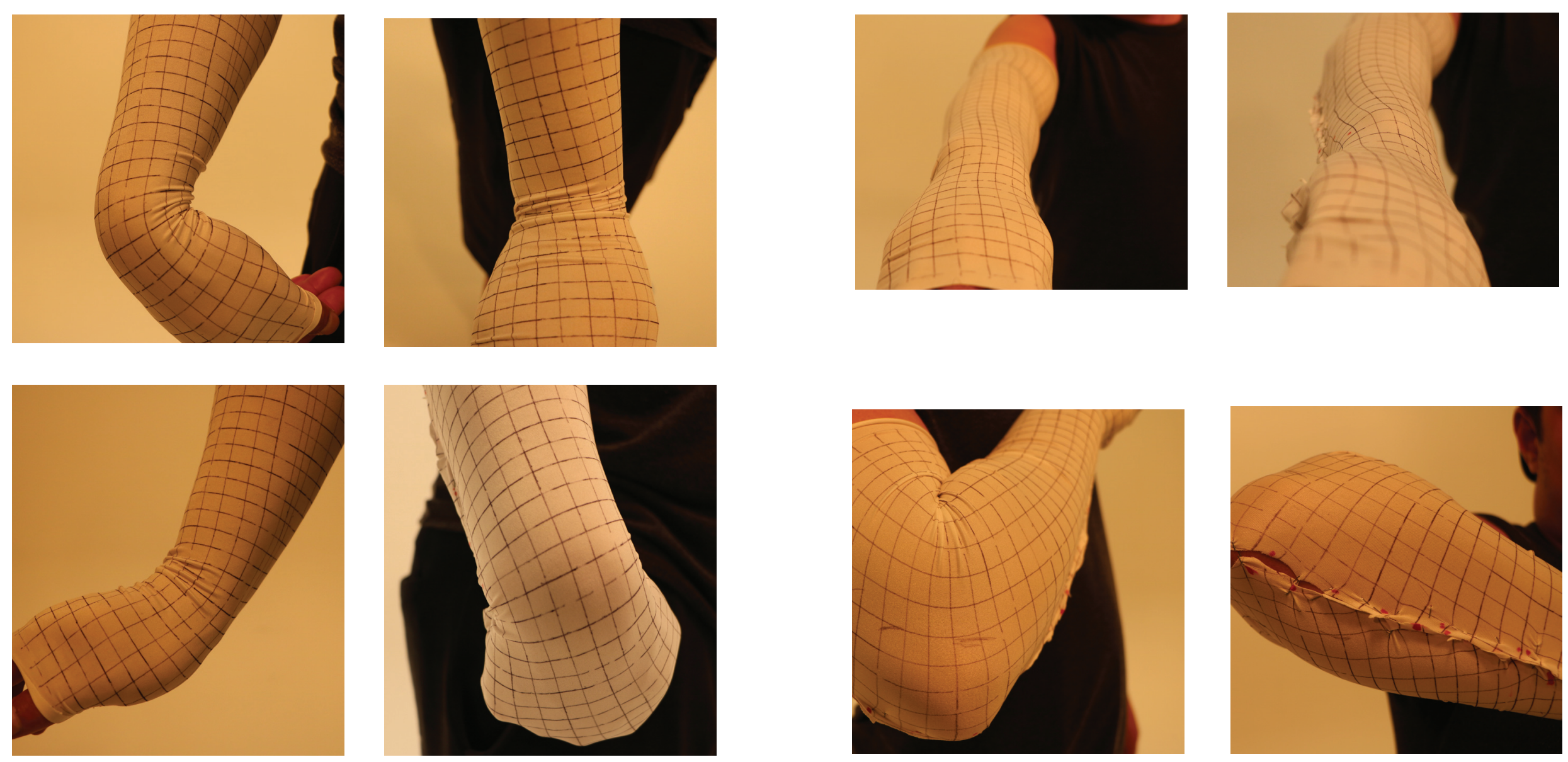

Figure 5.1

Photographs of the arm dynamic, focusing on the primary joins. 

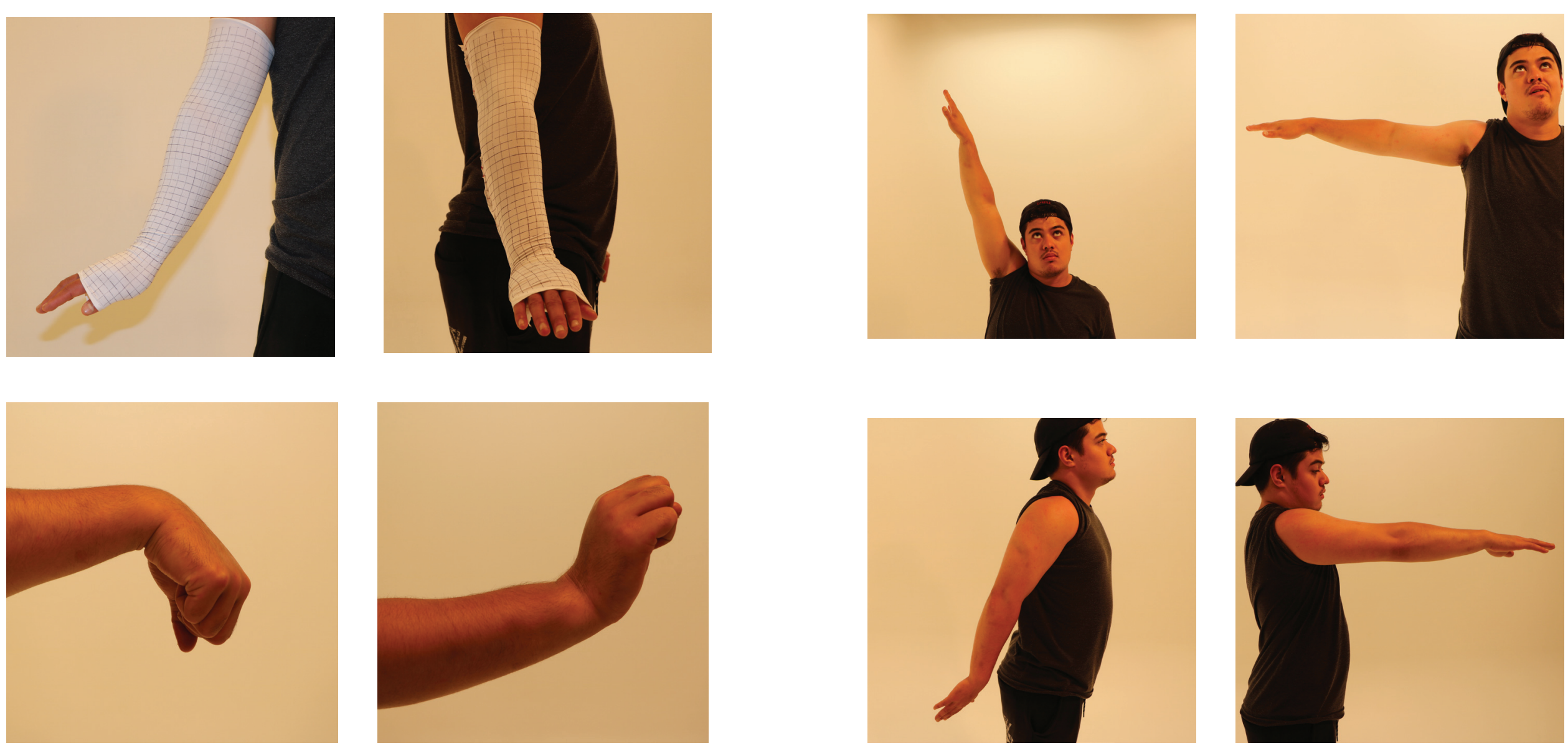

Figure 5.2

Photographs of the arm dynamic, focusing on the primary joins. 

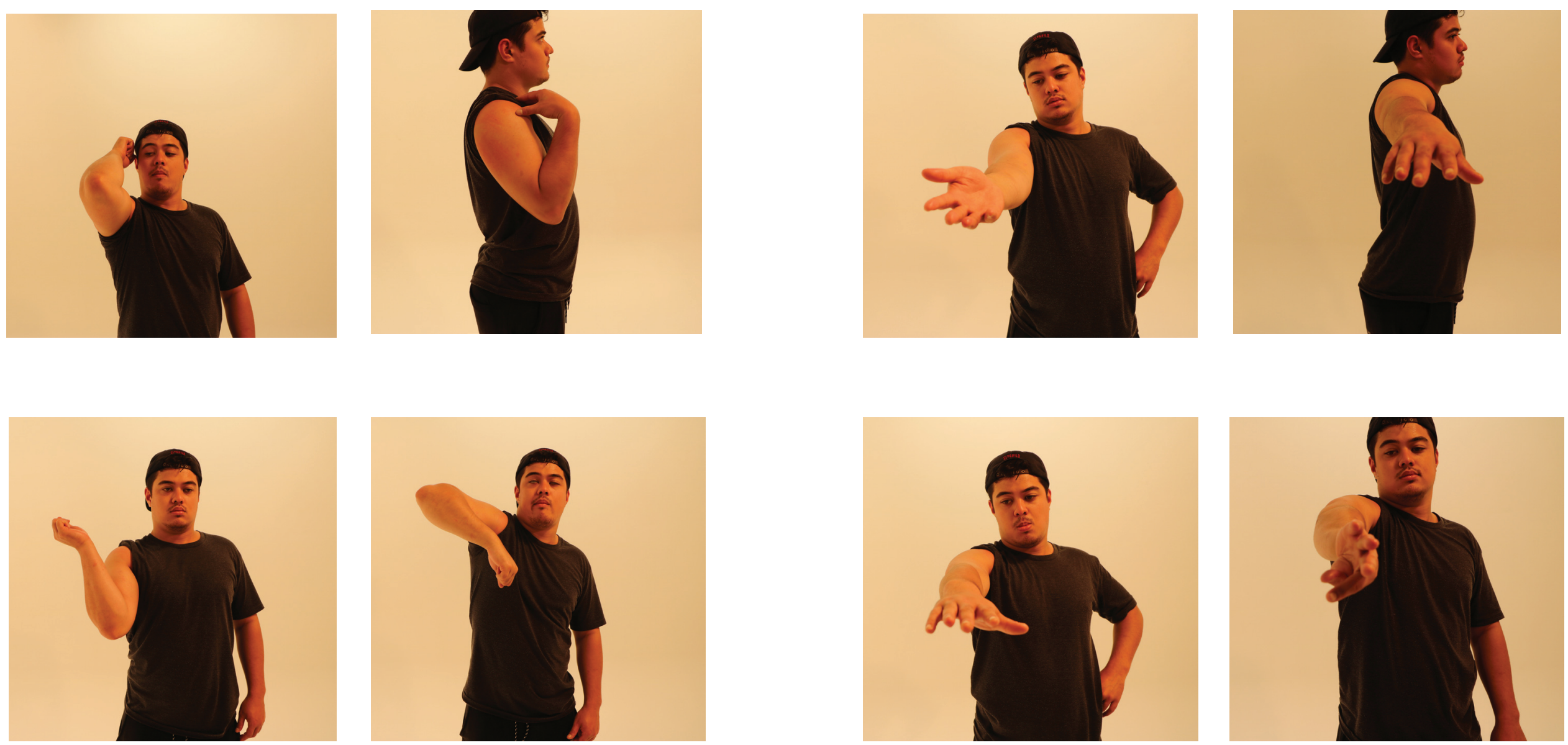

Figure 5.3

Photographs of the arm dynamic, focusing on the primary joins. 


\section{Battle Scars}

Chronophotography is a photographic technique used in the Victoria era which captures the motion of a moving object through a set of photographs. Chronophotography was originally utilized by scientists to study objects in motion, primarily humans and animals. On top of this it was also served purposes such as judging timed events and recording historical ones e.g horse races and studying movements of projectiles for war(Wikipedia, 2018). Using this photographic technique, understanding of the how, where, why, when, who and what will become apparent. Envisioning the athlete within the context through photography a protective hierarchy system can be created with in the design.Taking a series of photographs during a rugby tackle to analyse the natural responses of the deffender and attacker during physical contact. Determining which parts of the body are vulnerable to injury, through carrying out action which take place in the design context. From this the design challenge and how to respond to it relative to the design context can be achieved understood. 

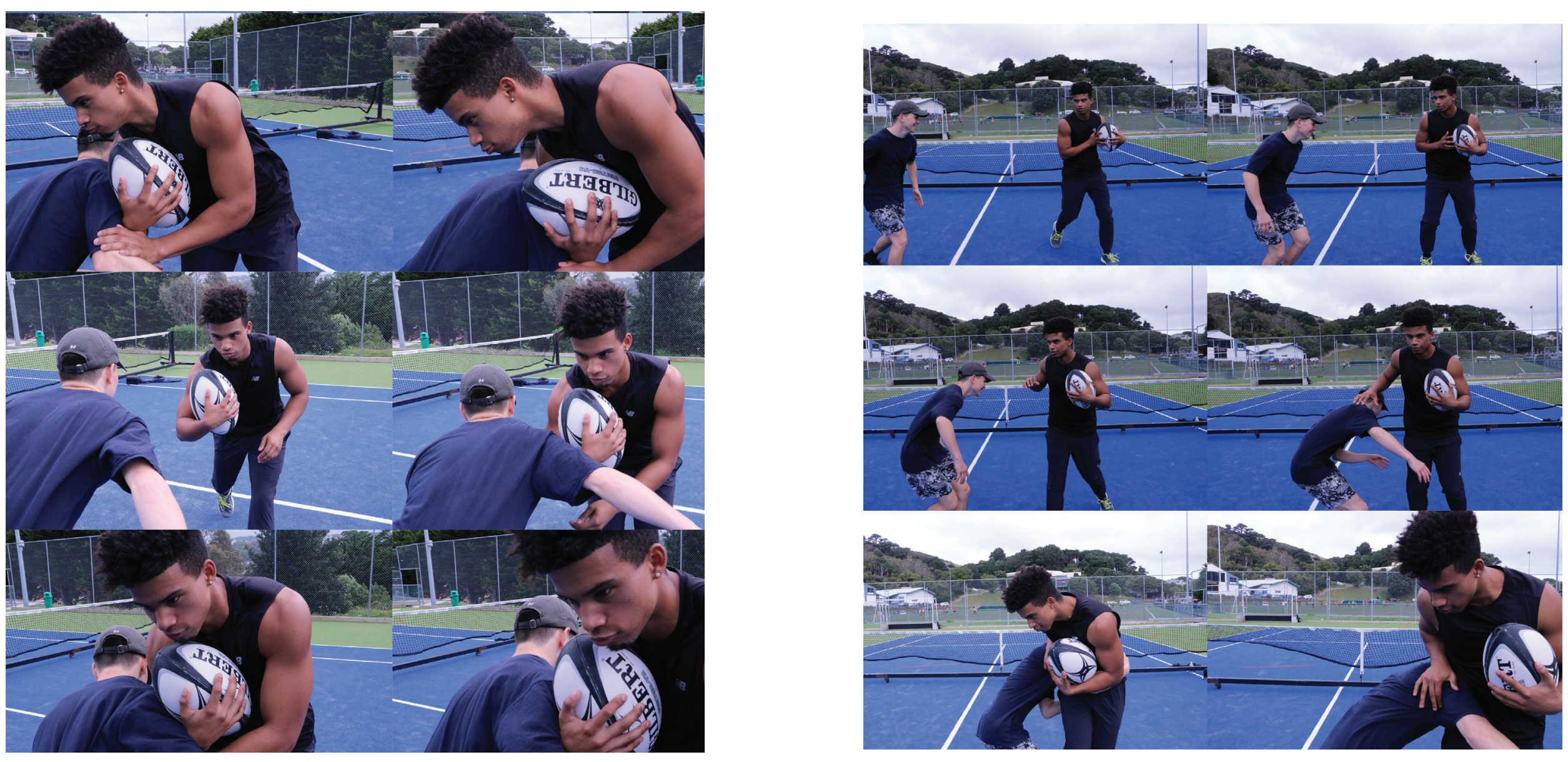

Figure 5.4

Photographs demonstrating the process of being tackled and how athletes respond to contact 

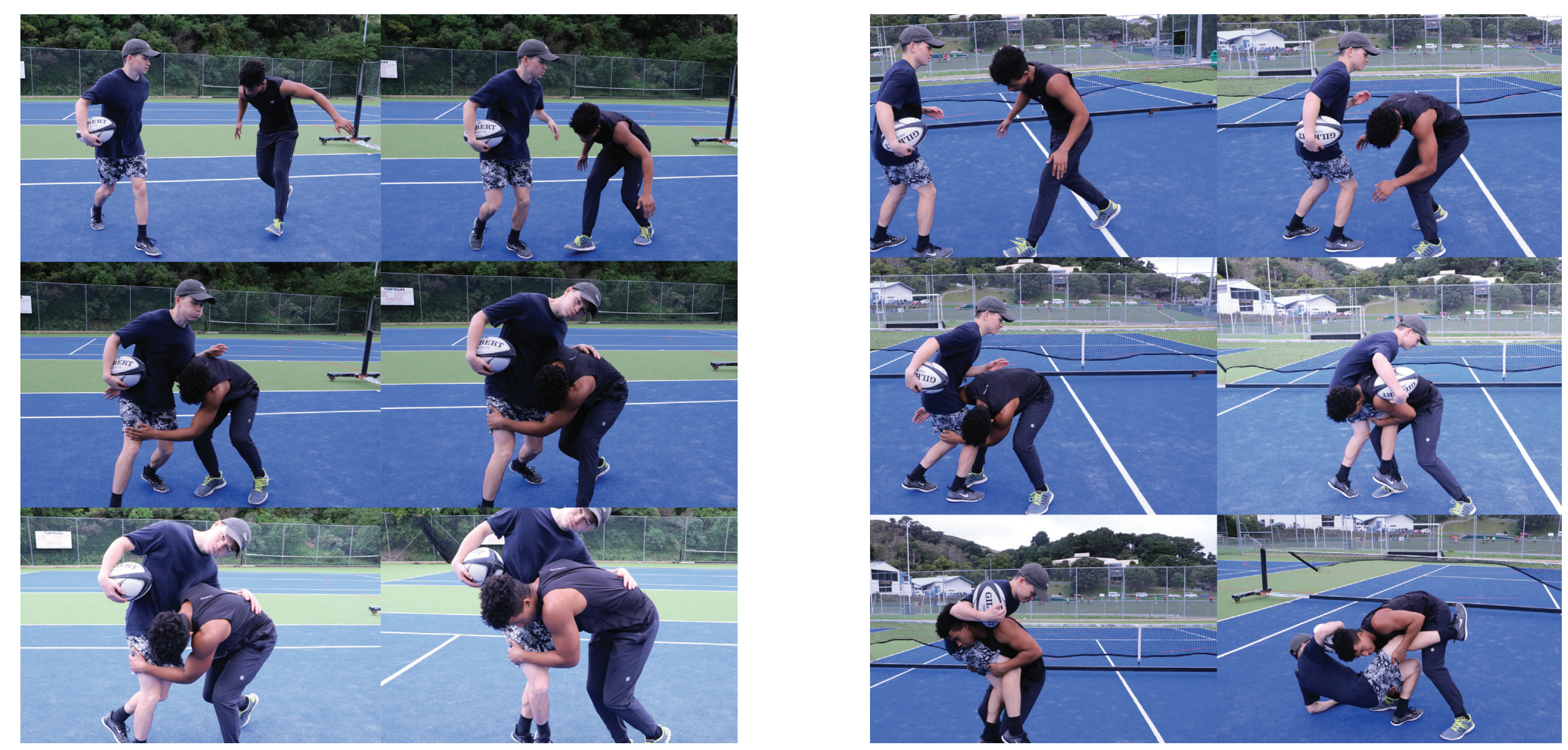

Figure 5.5

Evaluating which body parts are involved by the tackler and why 

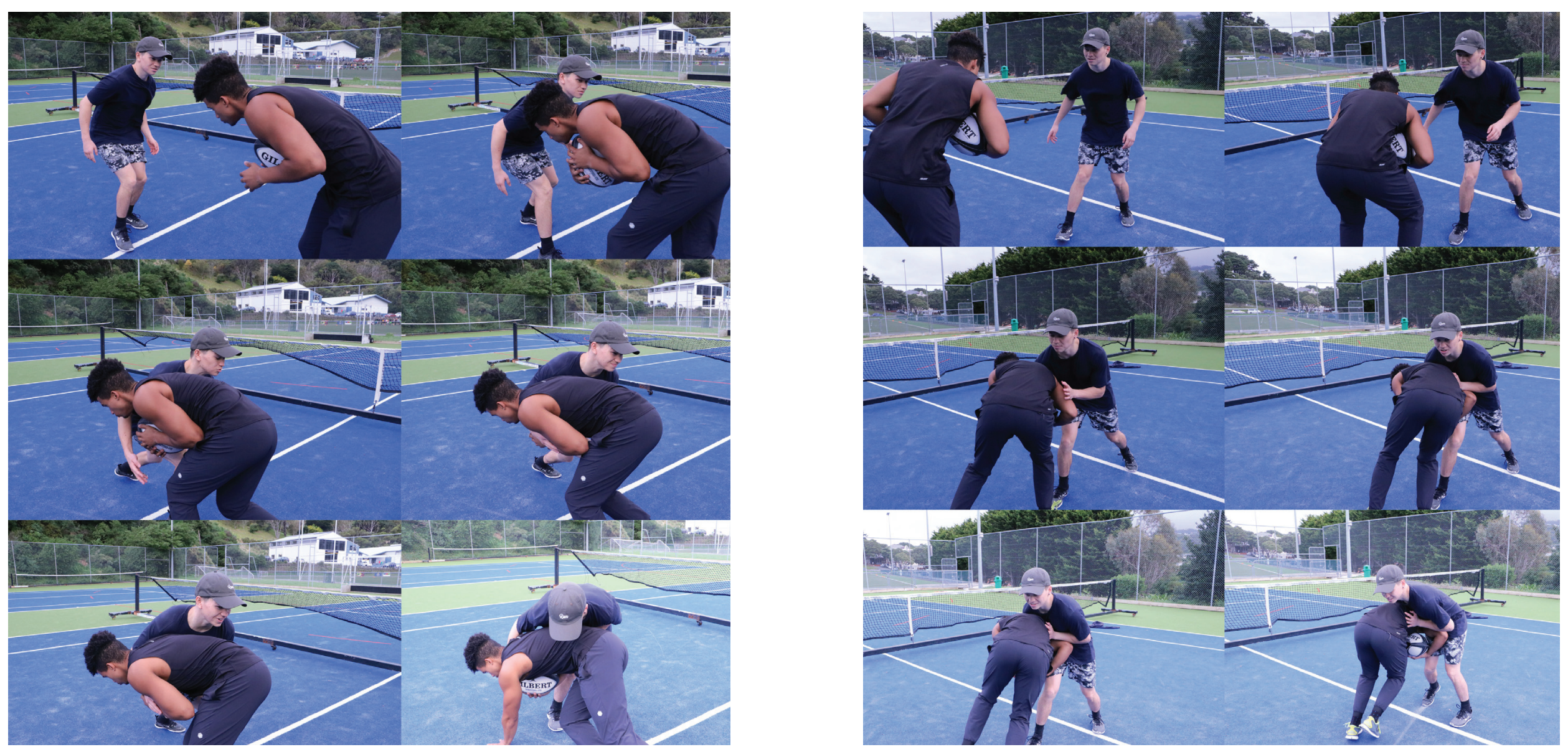

Figure 5.6

Demonstrating how the ball can be secured and protected during contact 

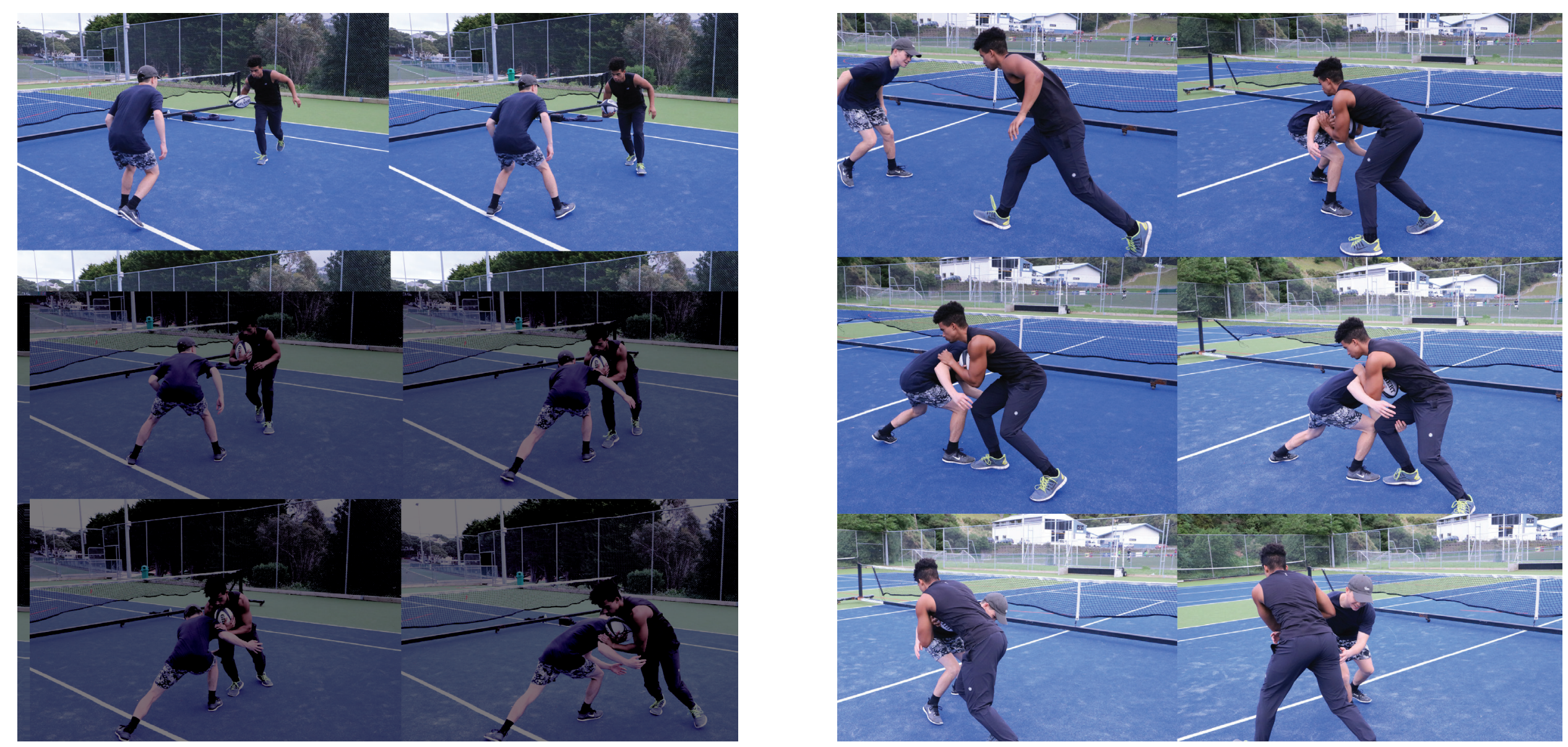

Figure 5.7

Showcasing which body parts are involved when defending from a tackle and why 

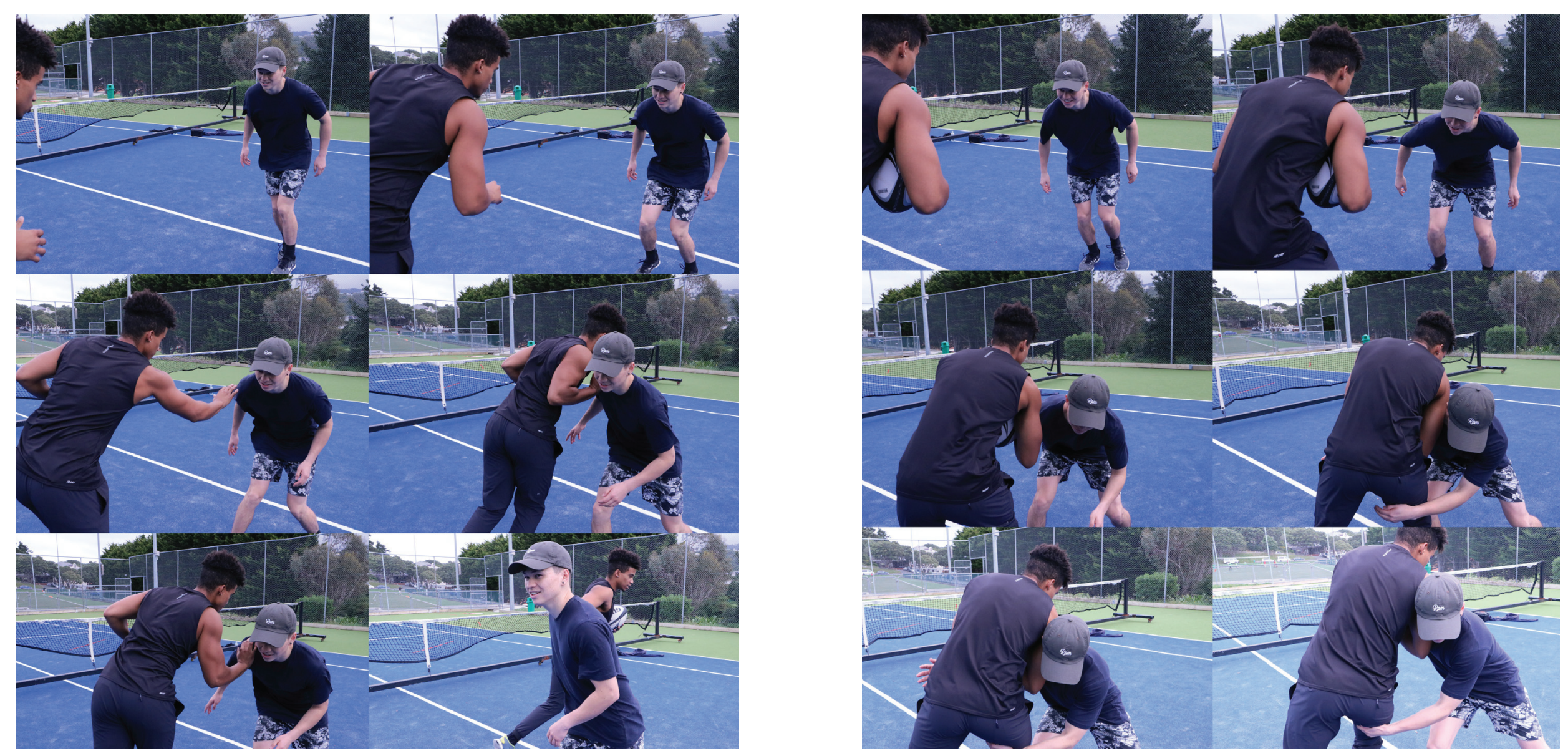

Figure 5.8

Showcasing which body parts are involved when defending from a tackle and why 

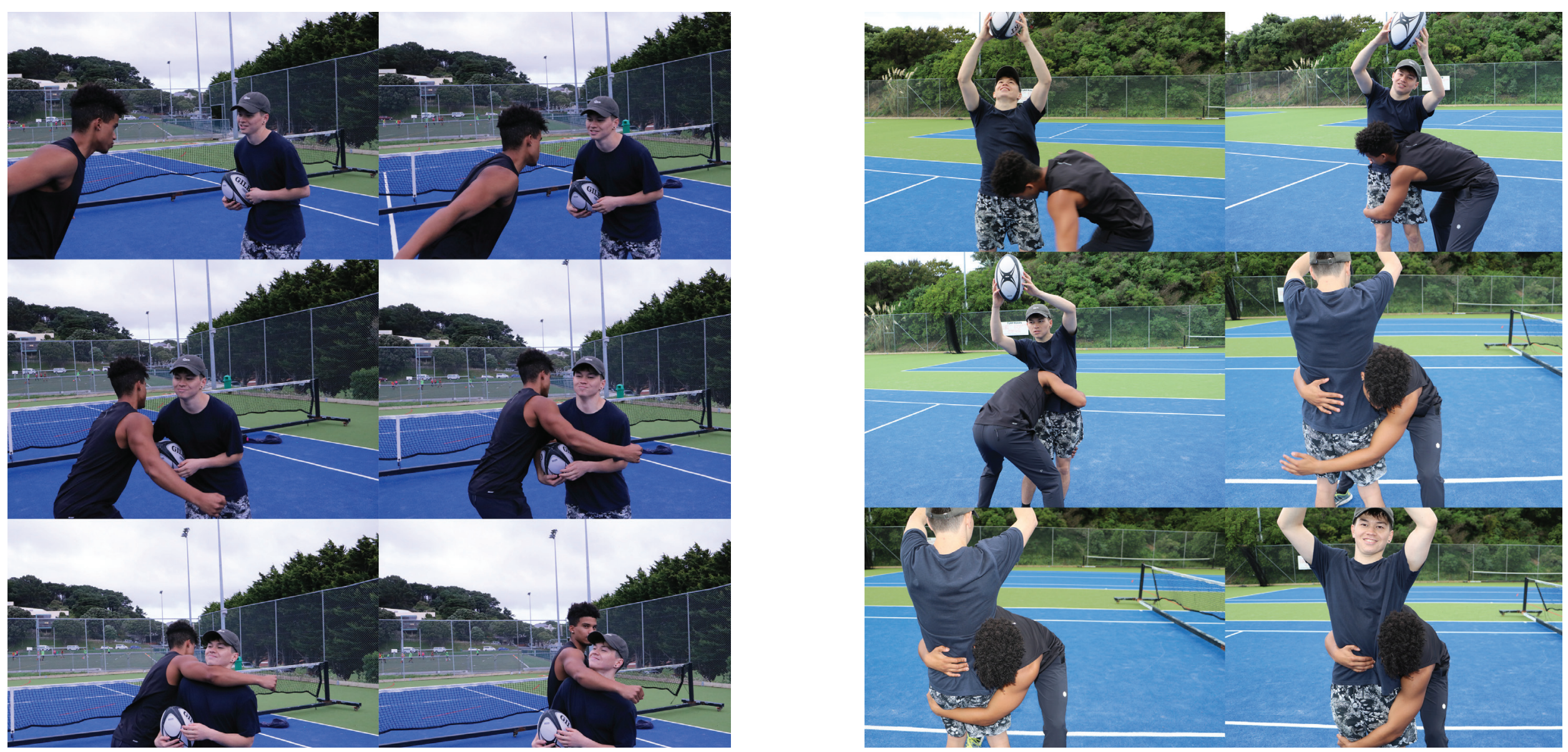

Figure 5.9

Investigating illegal tackles (left) and how the arms wrap around a the offensive player during a defenceless tackle (right). 


\section{Reflection}

From the studies carried out, findings allowed the design to be refined and design challenge to be approached in a practical manner. Through visual evaluation of the photographs, findings revealed the shoulders of the defender and the hips of the attacker to take most of the damage. However, body parts such as the triceps, bicep and forearm are also involved. The outside of the arm took heavy damage when to protecting oneself from the tackler, or protecting the ball. From this investigation a decision was made to focus heavily on the upper body, the shoulders and arms, as they are more prone injury due to their involvement with impact. As well as this, the upper body provides greater dynamic and therefore more design opportunities. From this the, upper body can be subdivided dependent on contact exposure as well as determining how much movement the skin needs to accommodate for. 

The digital creation phase embodies the strategies and techniques applied to create the resolved design model. Introducing $3 \mathrm{~d}$ scanning, $3 \mathrm{~d}$ modelling and other design applications that were utilized to fabricate the physical models from the digital workspace. Discussing the final stage of the design iterations and their contribution to the final outcome. Moreover, defining how to tailor the $3 \mathrm{~d}$ printed skin to the body and design context. 


\section{Digital Foundation}

To provide the $3 \mathrm{~d}$ modelling design with a foundation, $3 \mathrm{~d}$ scanning was utilized. The human body was scanned to provide the design approach with limitations, to ensure the speculative design outcome is justified. This was essential for the root/veins system, constraining the growth simulation to the surface of the $3 \mathrm{~d}$ scan. Furthermore, with $3 \mathrm{~d}$ scanning the final outcome can be customised to a specific body and provide the appropriate fit. Digital scales can be generated based on the given geometry and therefore provide effective dynamic movement. To produce the final design a full upper body $3 \mathrm{~d}$ scan was required, this was done by cleaning and duplicating one half. Common among $3 \mathrm{~d}$ modelling, a symmetrical mesh is mirrored to reduce the workload, this technique was applied to the $3 \mathrm{~d}$ scan.
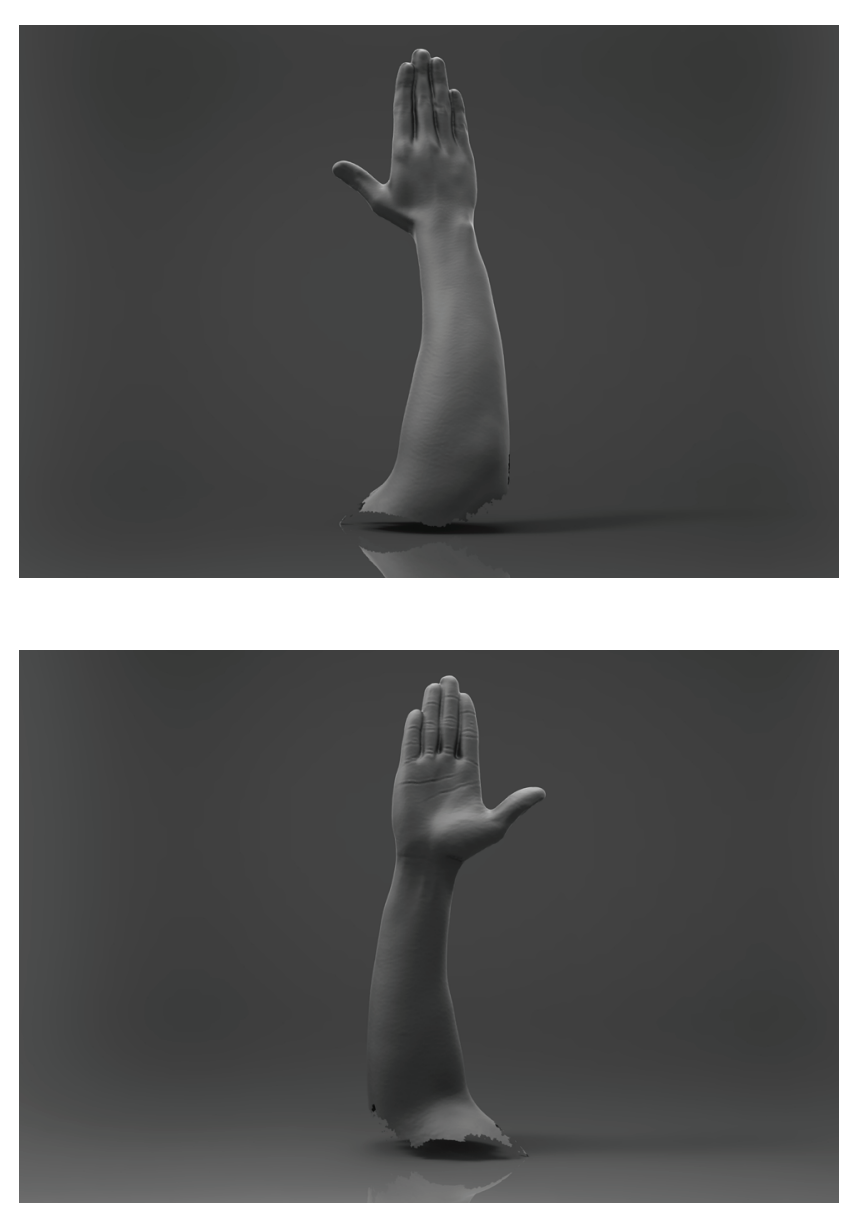

Figure 6.1

Initial $3 \mathrm{~d}$ scan during and for the early design stages. 

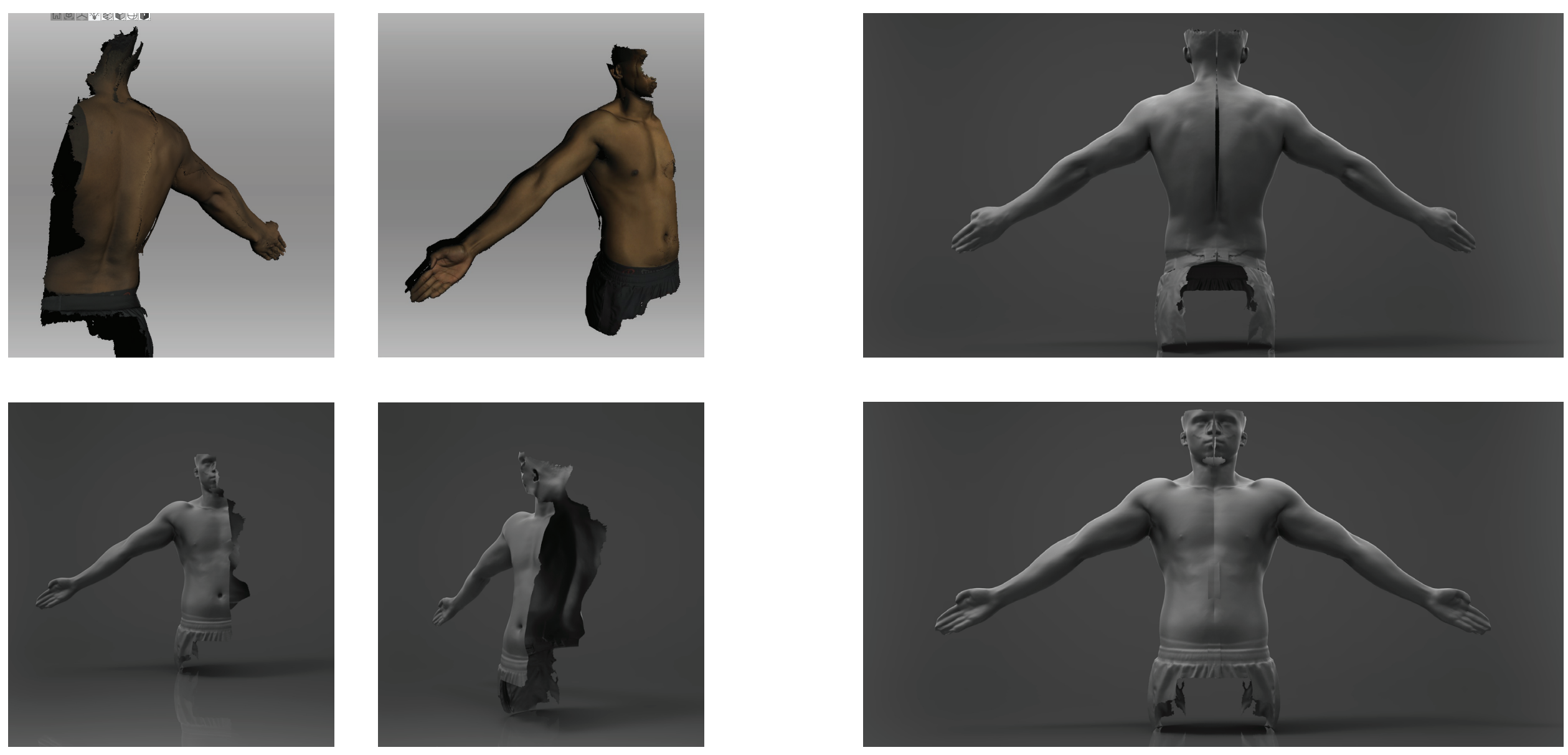

Figure 6.2

Demonstrating how the final $3 \mathrm{~d}$ scan was edited to cater for the design process. 


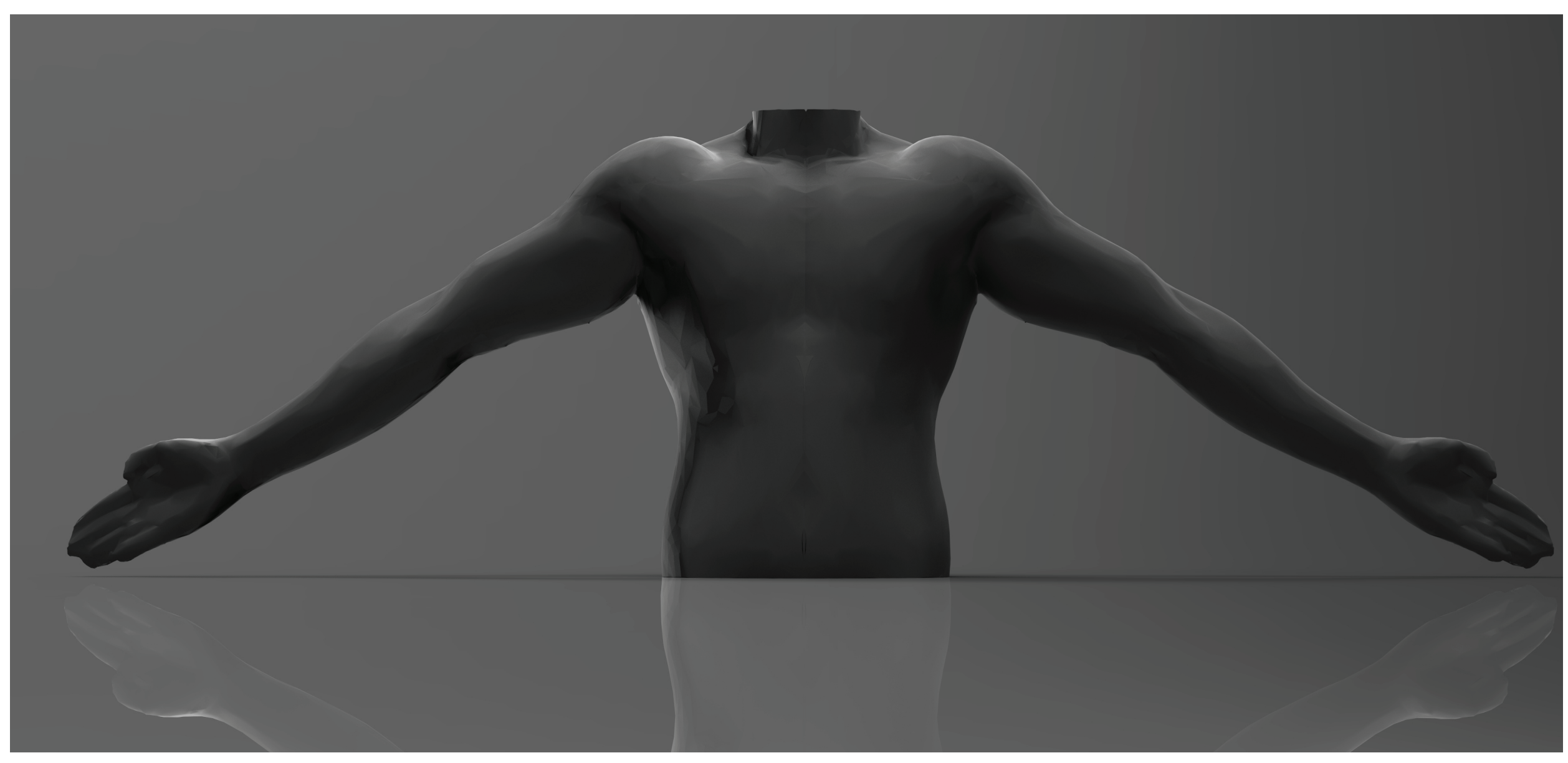

Figure 6.3

Completed upper body scan. 


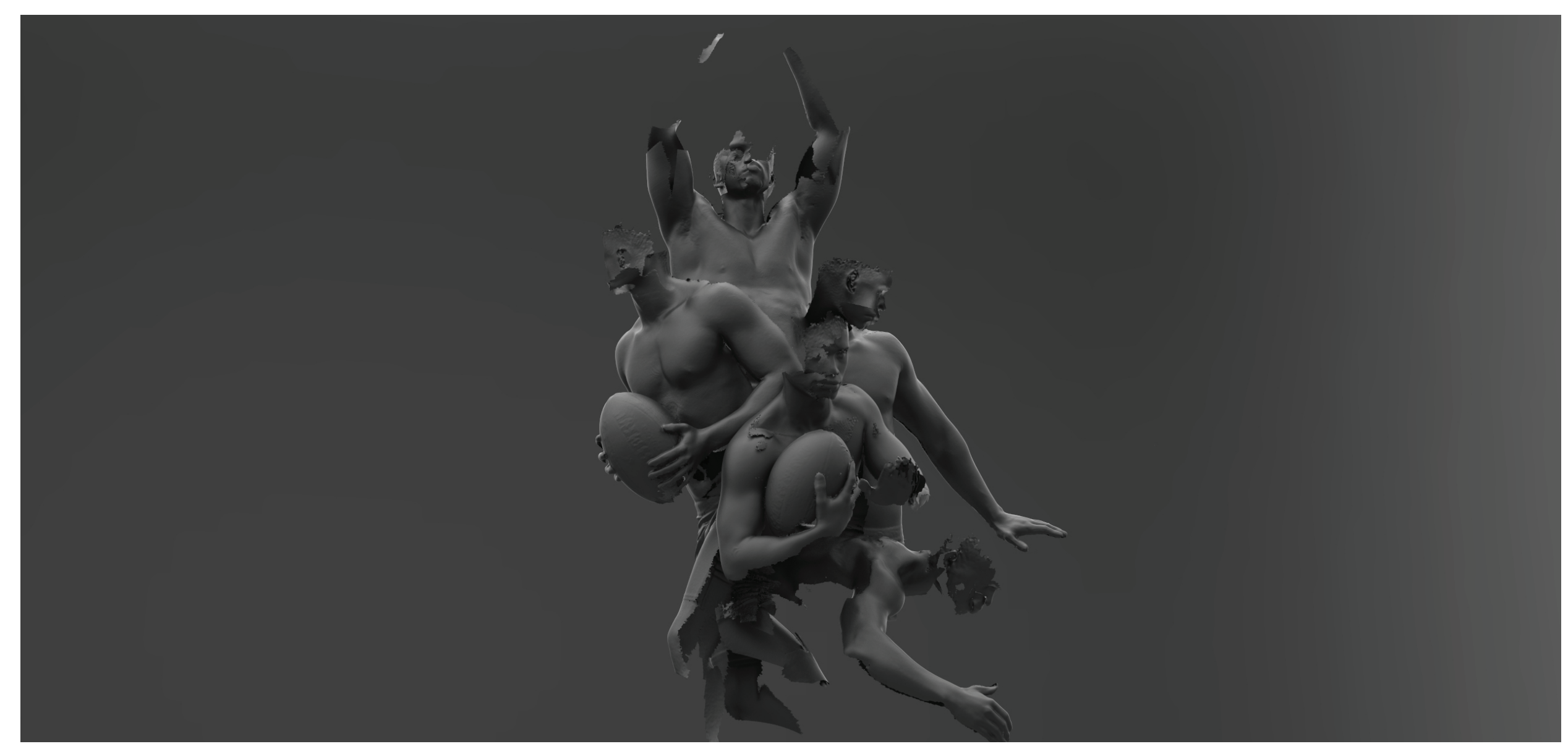

Figure 6.4

Creative composition of dynamic $3 \mathrm{~d}$ scans based on rugby actions. 


\section{Digital Growth}

Using the 3ds max, a protective root and scale system was designed through an iterative design process. By using the powerful particle system in 3ds Max (particle flow) a protective skin was grown within the digital workspace and on the surface of the $3 \mathrm{~d}$ scan. Particles were generated through a set of coding commands to create the desired outcome. Assuring the aesthetic is organic and root-like to confirm the skin is perceived as a growing network. From this, the system is intended to commemorate selfgrowth and identity, with the intention of enhancing sports performance through motivation and confidence.

Digital scales were developed from the polygon surface geometry and altered for efficient functionality. Moreover, creating a protective skin from the athlete's digital skin ( $3 \mathrm{~d}$ scan). Therefore, the underlying design principal was to design from the body instead of to the body, to produce a meaningful design outcome. 

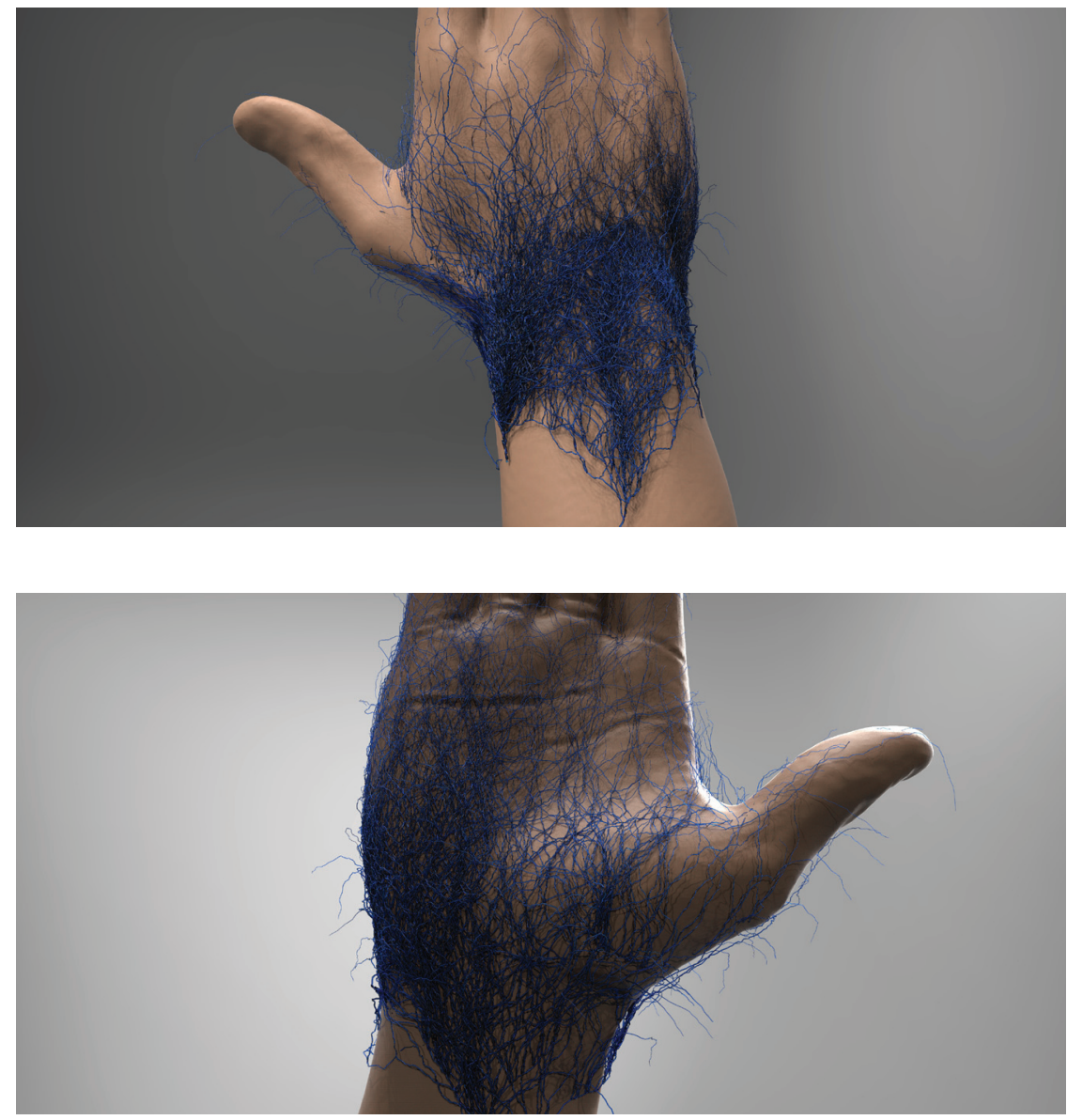

Figure 6.5 

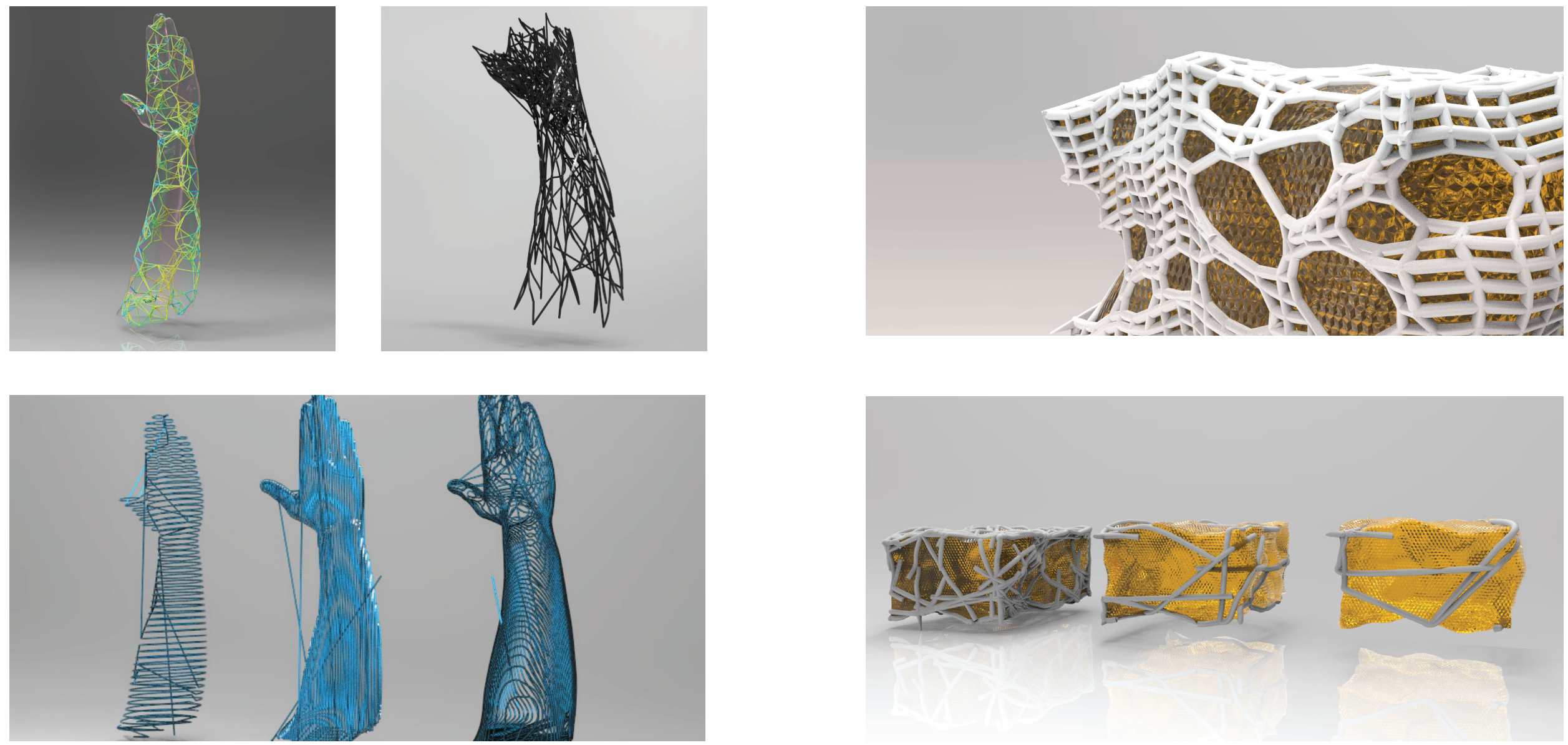

Figure 6.6

Early design iterations based on several design strategies, for the growth system 

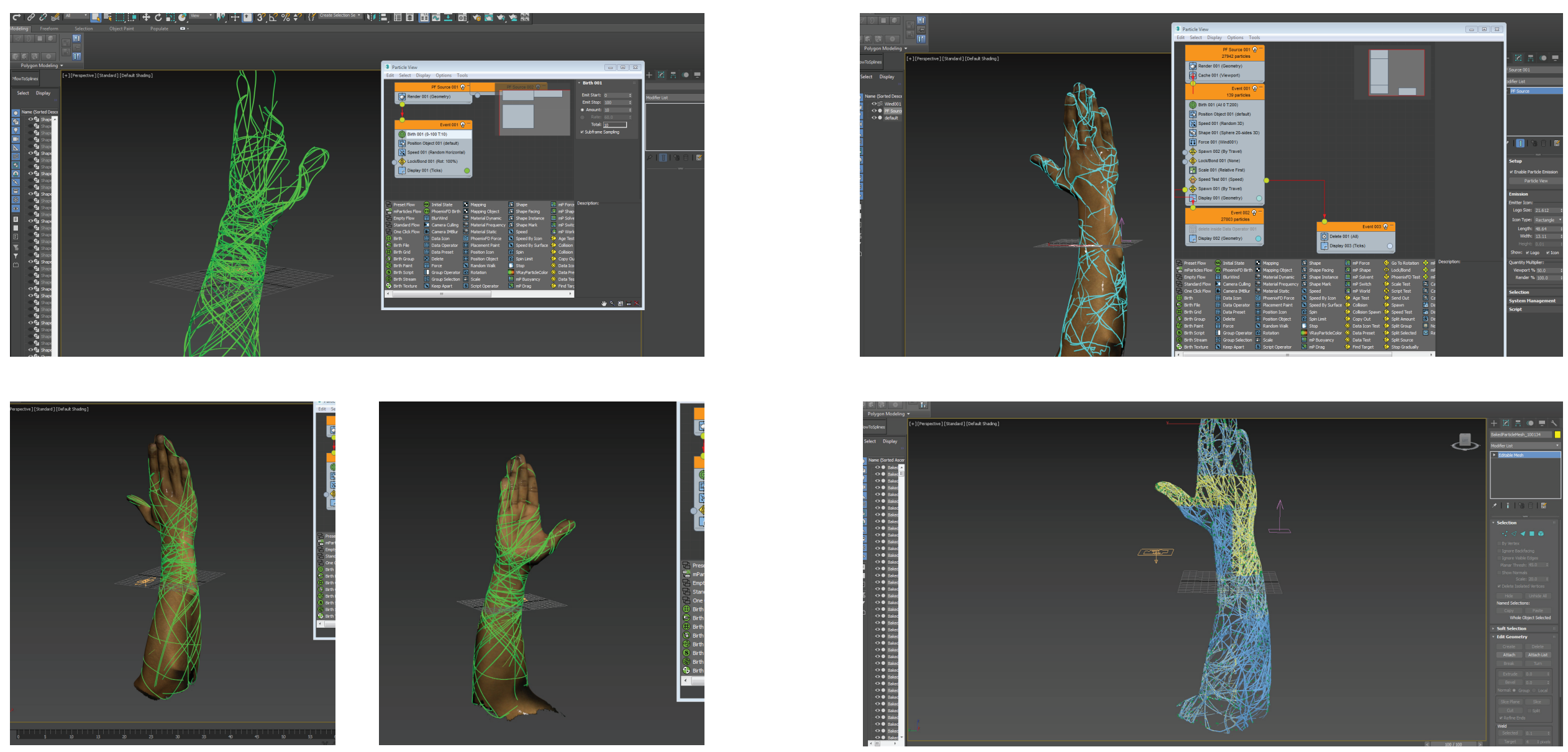

Figure 6.7

Insight to the digital process behind designing the root/vein protective system. 

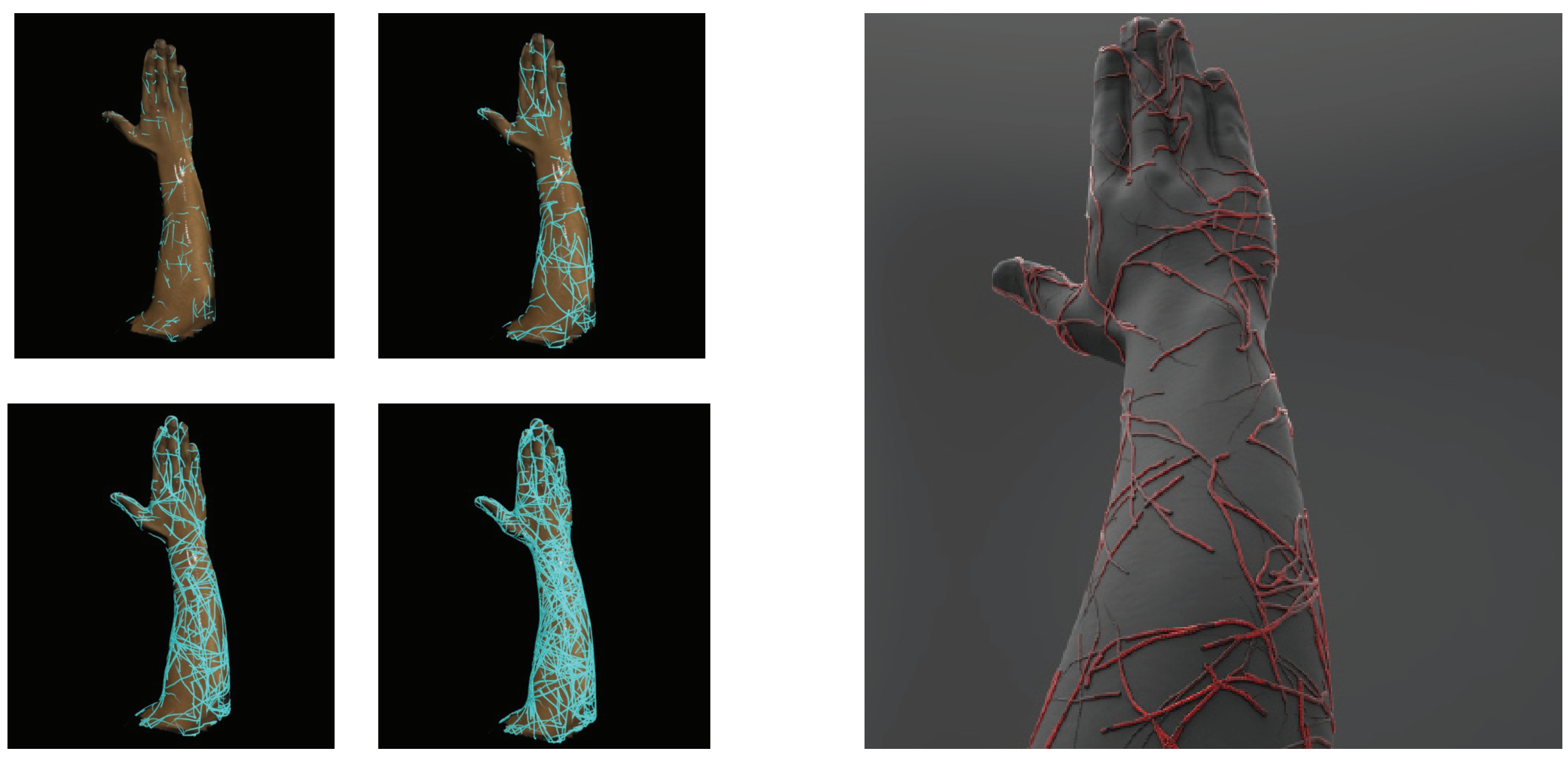

Figure 6.8

Resolved method for the generative structural support system. 


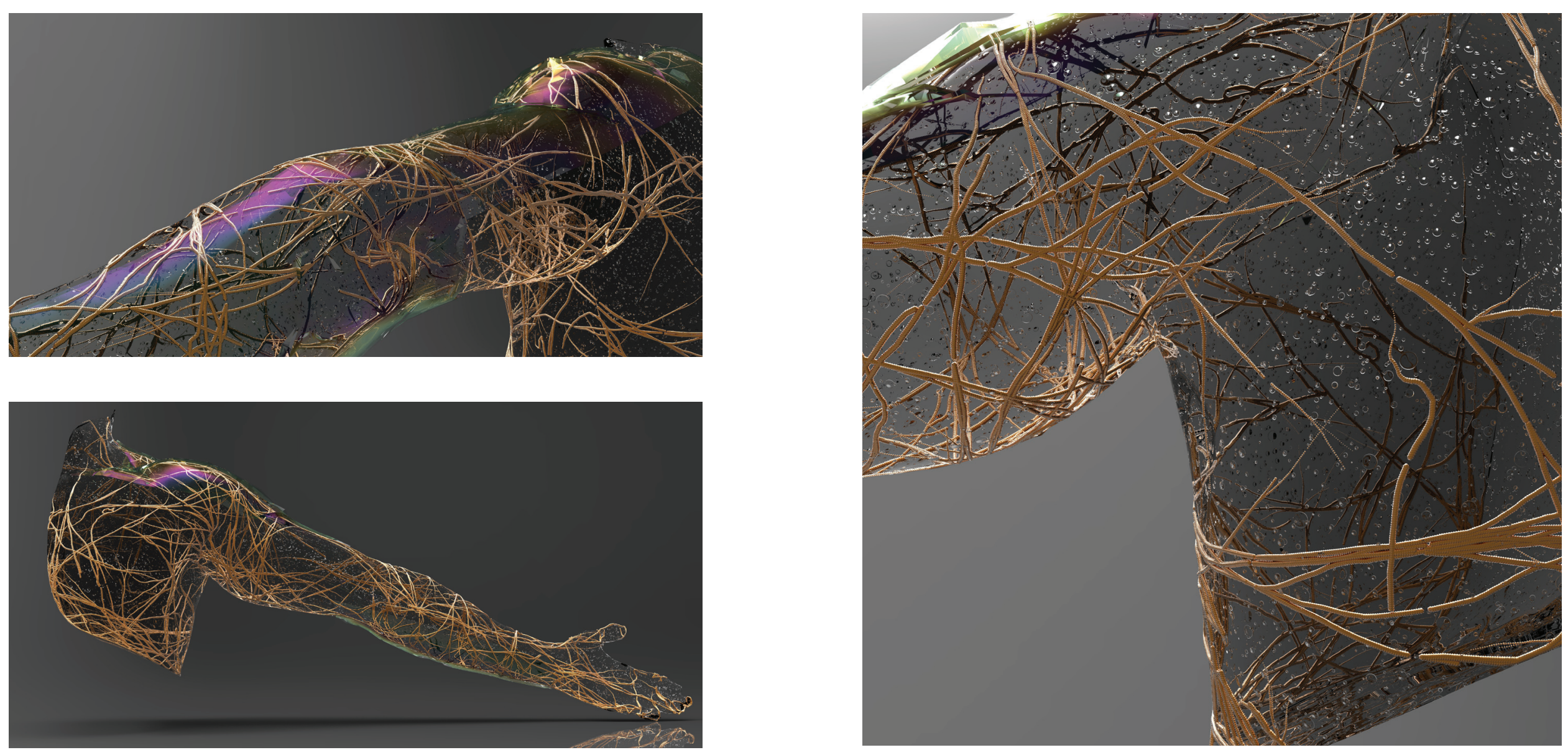

Figure 6.10

Renders visualising the growth system as if inside the body to determine its compelling design qualities. 

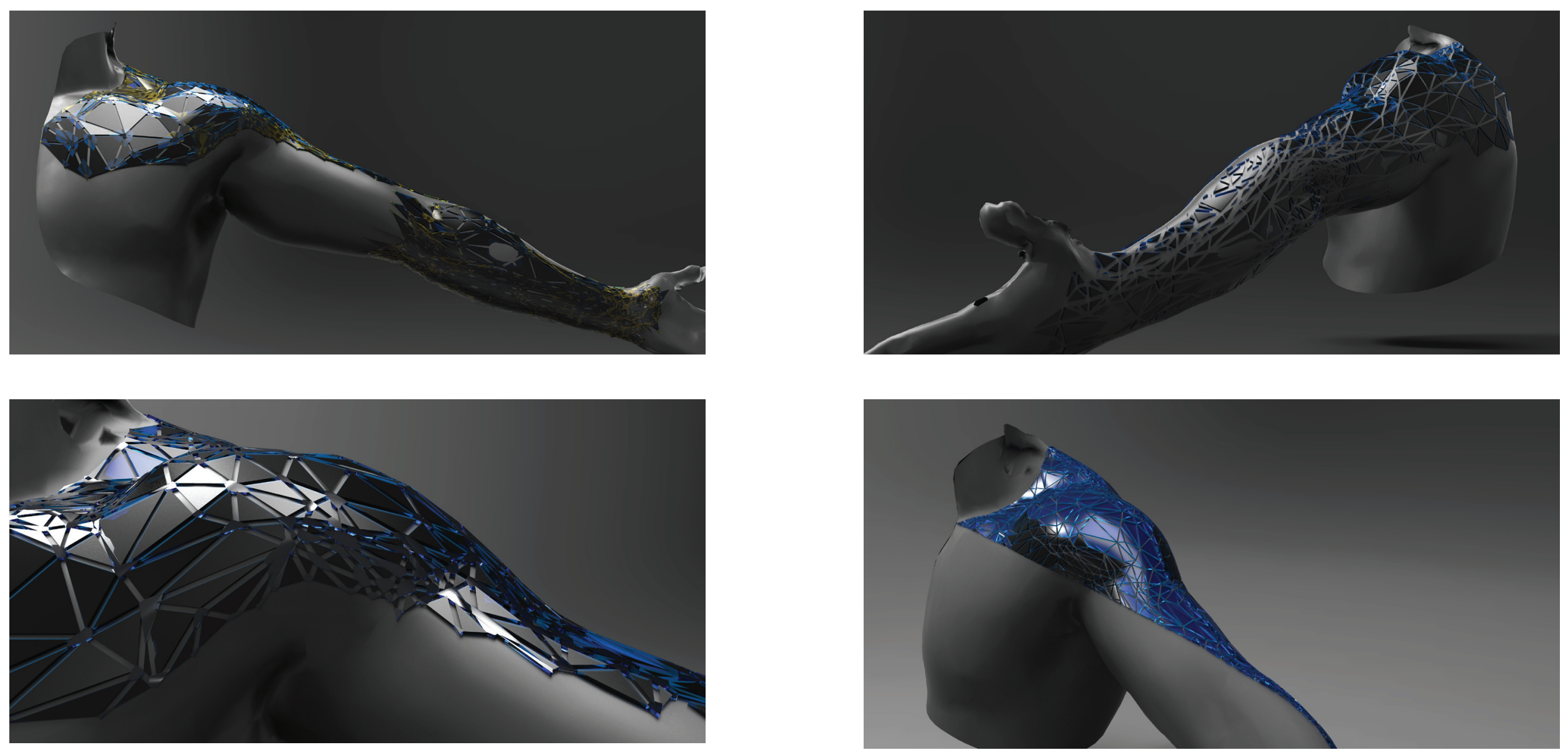

Figure 6.11

Showing scale configurations on the body and communicating the conformal capabilities. 


\section{Tailored for Protection}

Based on the 'battle scars' experiment the $3 \mathrm{~d}$ scanned models were subdivided depended on which areas of the body are involved in a rugby tackle. Predominantly focusing on the upper body and the parts exposed

to impact. The human geometry was categorised to facilitate protective hierarchy. Furthermore, considering how these segments are composed to create dynamic flow for efficient movement during the sport. 

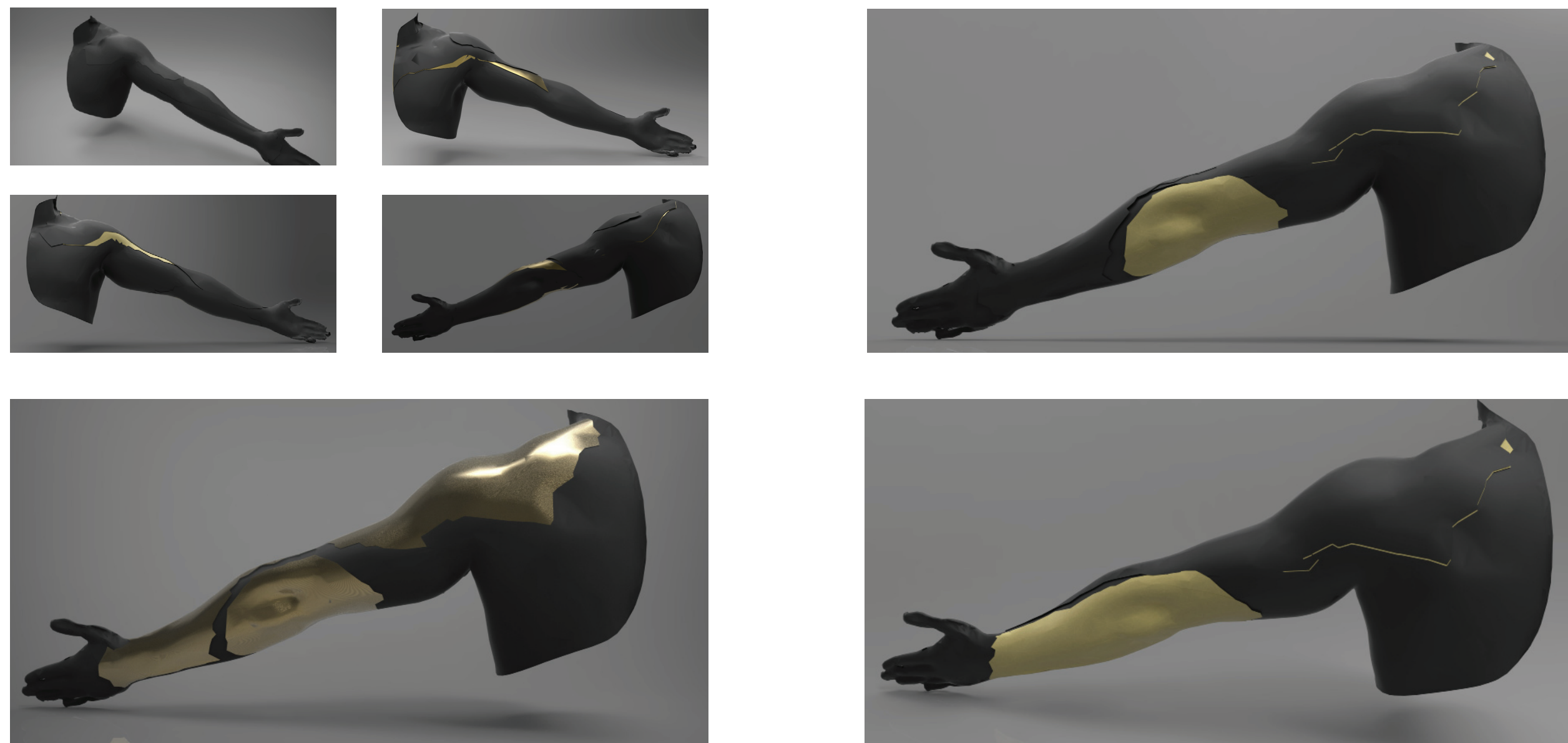

Figure 6.12

The arm is sectioned for protection, determining how the vulnerable body parts can be protected from impact 

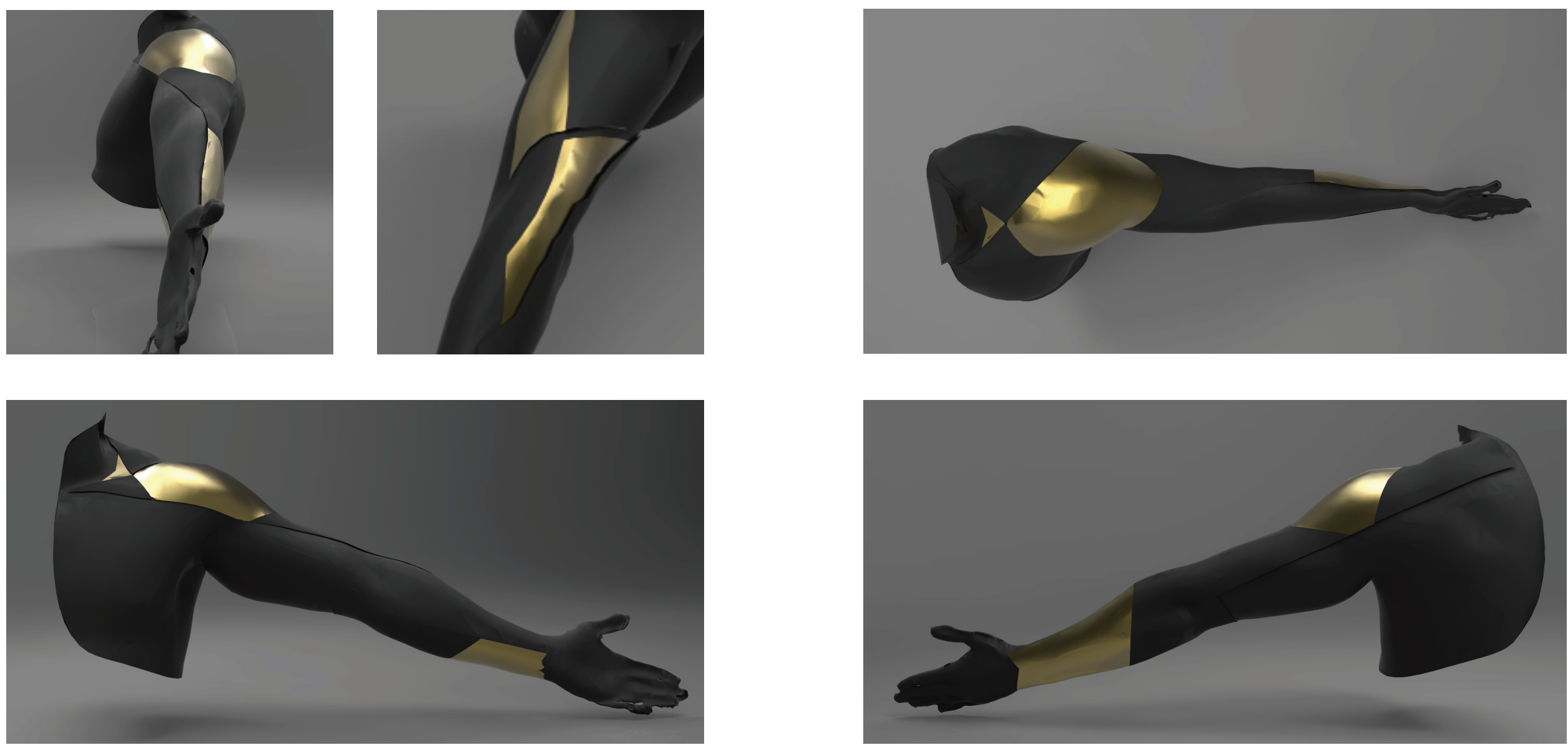

Figure 6.13

Establishing where to protect the body in regard to 'Battle scars' investigation findings 

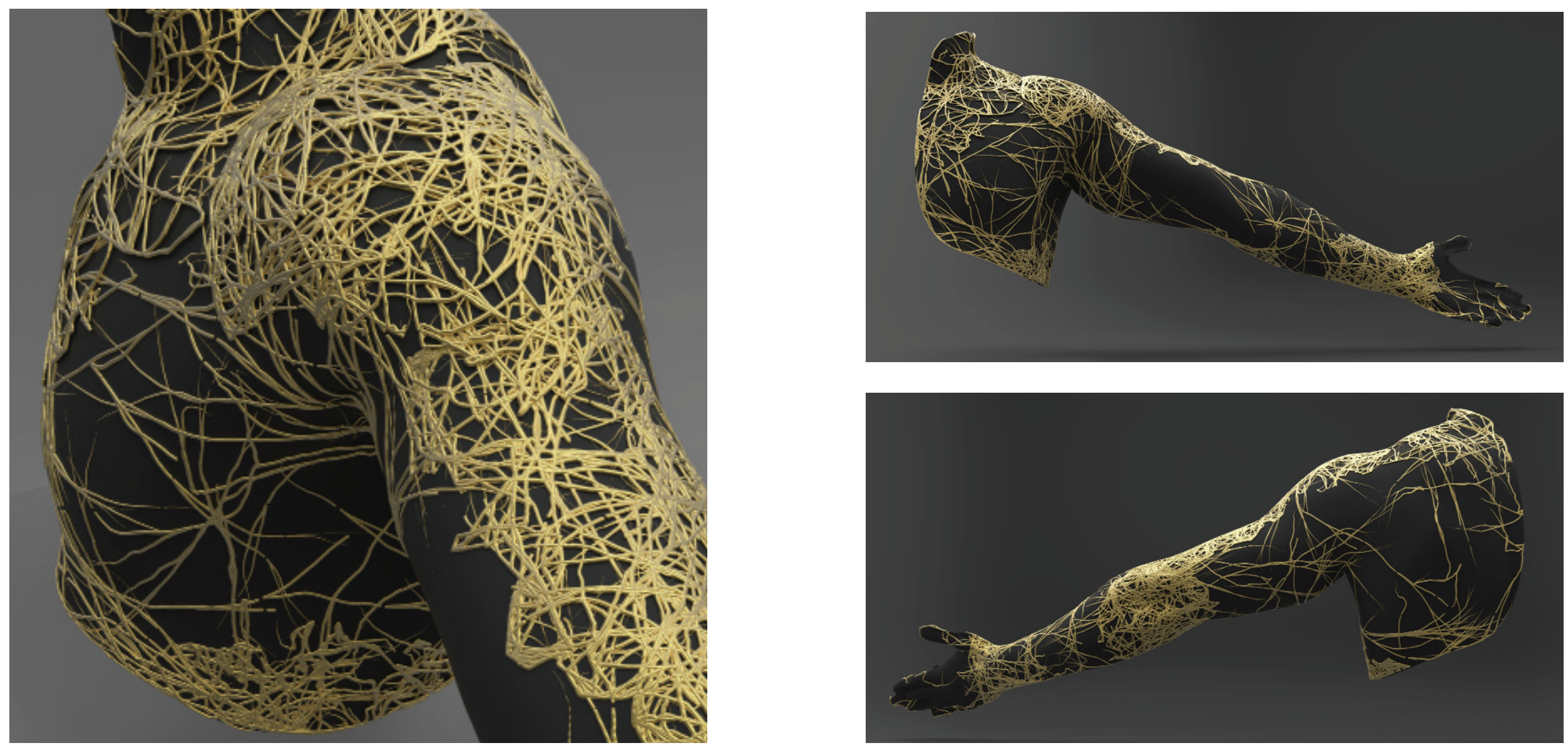

Figure 6.14

Controlling the supportive vein system for a defined design output.

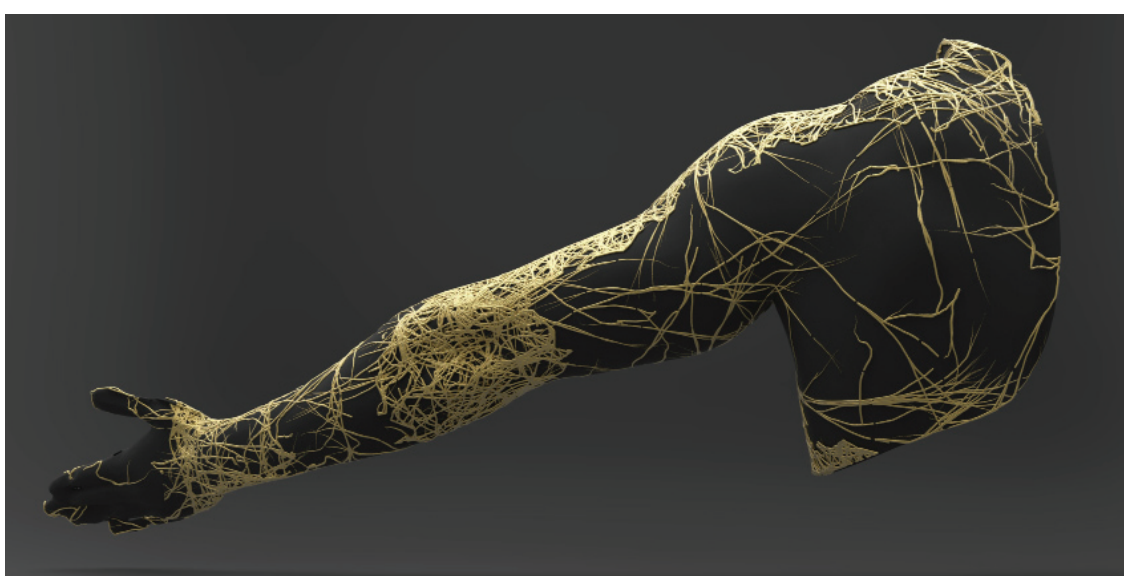




\section{Final Design layout}

To provide an ergonomic fit to the body the padding structure was changed as the previous subdivided layout was too impractical. When tested its ability to conform and flow with the body at a greater thickness was very poor. The reasoning behind this is because the $3 \mathrm{~d}$ printed area was too large and therefore lacked the basic functional qualities needed to ensure an ergonomic fit while allowing for dynamic movement. The final design subdivision was not only based on the muscle groups and area of impact but also considered how flexible the design outcome needs to be to enable comfort and dynamic movement.

Finalised

Therefore, the final padding involves a rectangular panelling which follows the contours of the body. This design will allow the physical model to wrap more effectively assuming the fabric is stretched in the right direction. By simplifying and refining the basic form of the design, the overall functionality of the wearable is improved efficiently. On top of this, the aesthetic and function of the design is as protective bandage. Furthermore, being able relate the design back to primitive yet effective protective strategy, provides a sense of credibility and through this a compelling design. 

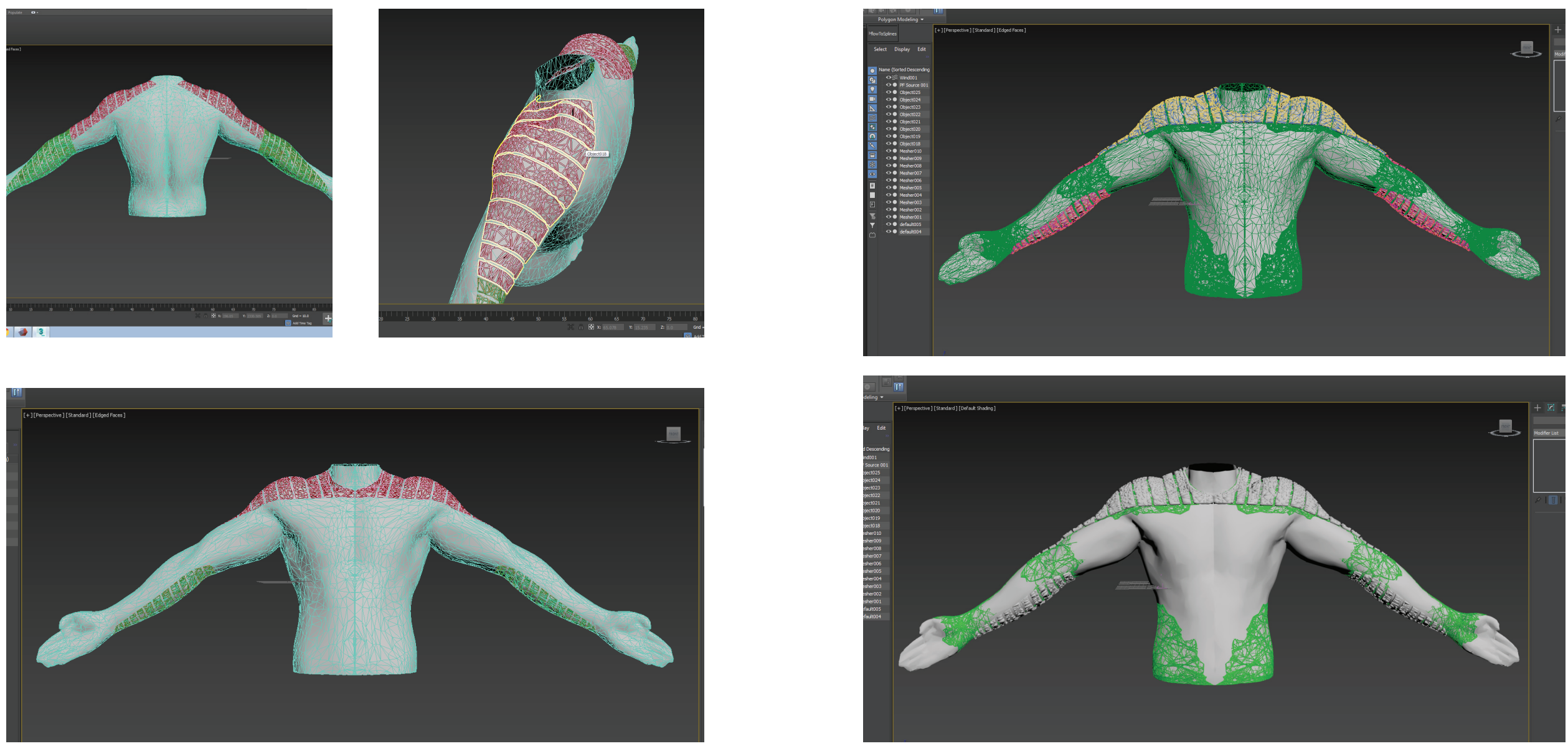

Figure 6.15

The resolved $3 \mathrm{~d}$ model with in the digital design space. 


\section{Virtual to Reality}

To enable the $3 \mathrm{~d}$ models to be printed onto the chosen textiles, these $3 \mathrm{~d}$ forms needed to be flattened. This was done through using the UV unwrapping. UV unwrapping allowed the $3 \mathrm{~d}$ mesh to be unfolded, this process commonly used in $3 \mathrm{~d}$ modelling. As the polygons that make up the $3 \mathrm{~d}$ model can be flattened and painted and then reapplied to the surface of the $3 \mathrm{~d}$ object. However instead of using the UV map for painting or texturing purposes, the aim is to utilise this technique allowing the $3 \mathrm{~d}$ models to be printed onto the chosen textiles using UP Box $3 \mathrm{~d}$ printer. By doing this Polygon figuration will provide accurate coordinates that will allow the shape to be transformed into its original three dimensional shape post $3 \mathrm{~d}$ printing.

When using the UV mapping modifier in 3ds max, there was difficulty exporting the UV map to real world dimensions. Therefore, Meshmixer was used as a substitute, by importing an $\mathrm{OBJ}$ section with the consideration of the UP Box 3d printing bed dimensions 250 X210. From this using Meshmixer's Unwrap feature the model was able to be flattened. The unwrapped surface was then exported as a SVG and imported into Adobe Illustrator. Using Illustrator the file was cleaned and converted into a vector. Using Adobe Illustrator the cleaned file was exported as DXF. This was then imported into a $3 \mathrm{~d}$ modelling program called Fusion 360, using Fusion the file was able to be imported with the correct dimensions and units. Furthermore, allowing the DXF file to become three-dimensional through using the extrusion features. 

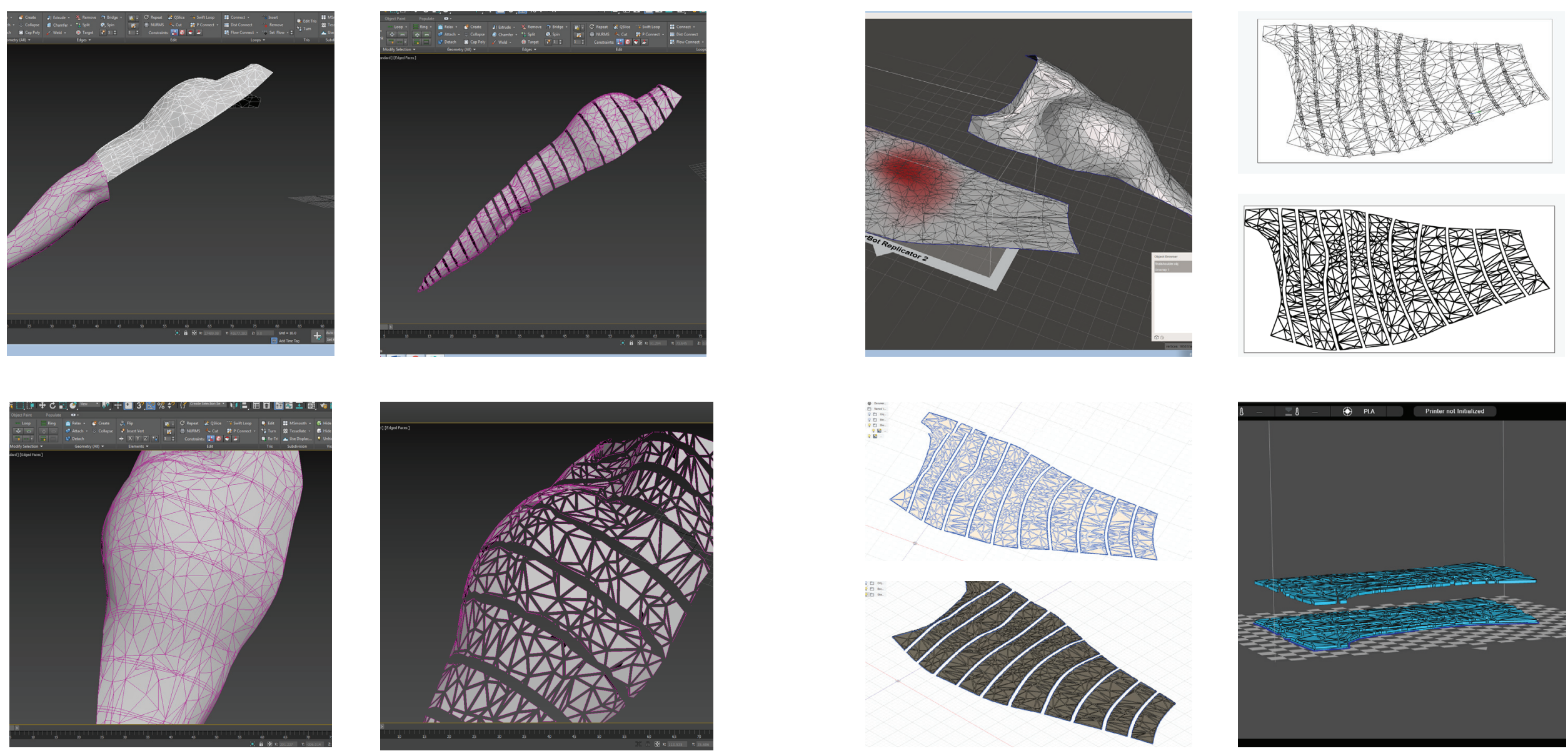

Figure 6.16

The process of creating a printable resolved 3 model 

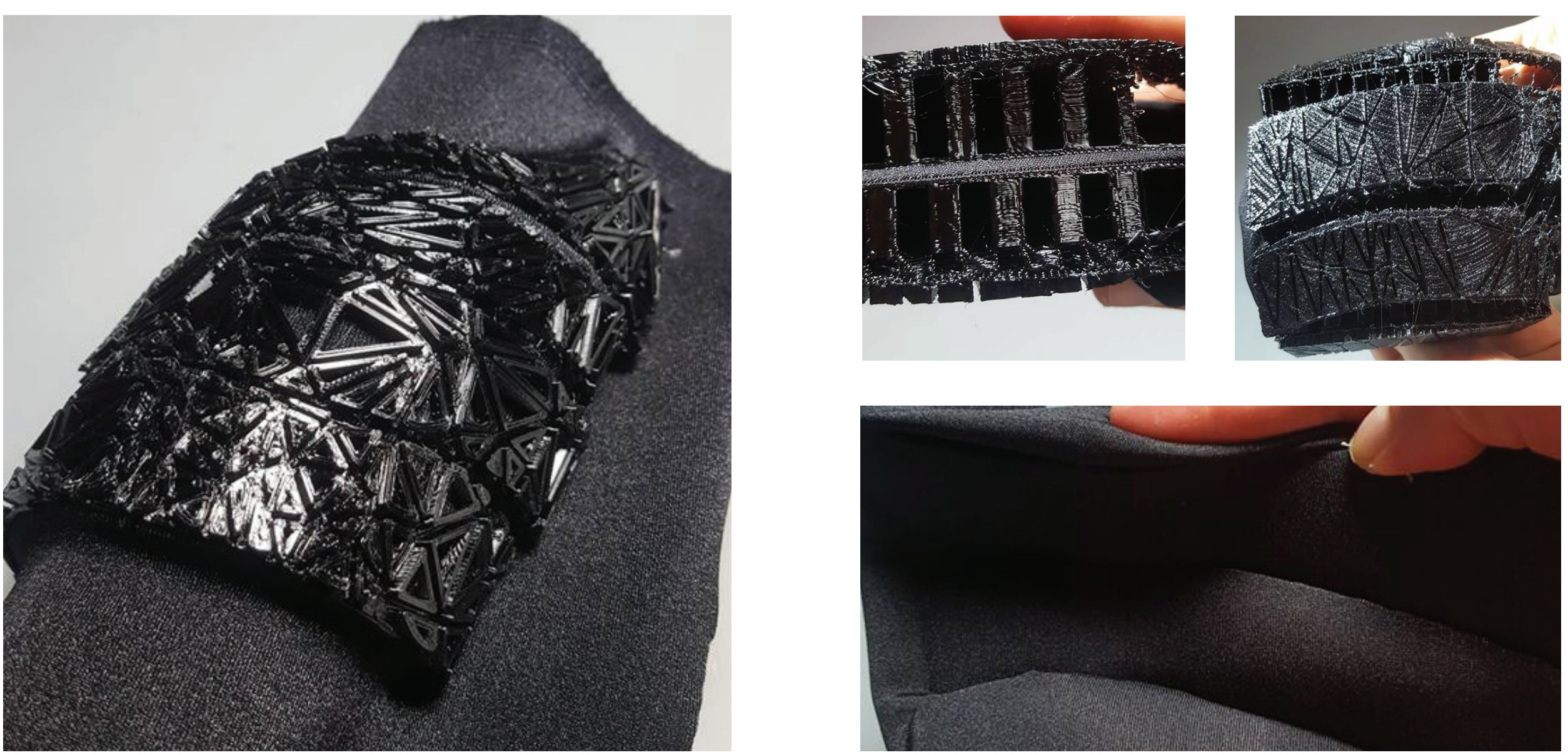

Figure 6.17

Final protective padding prints qualities, assessing how well impact is absorbed, how the design contour based on the direction of tension

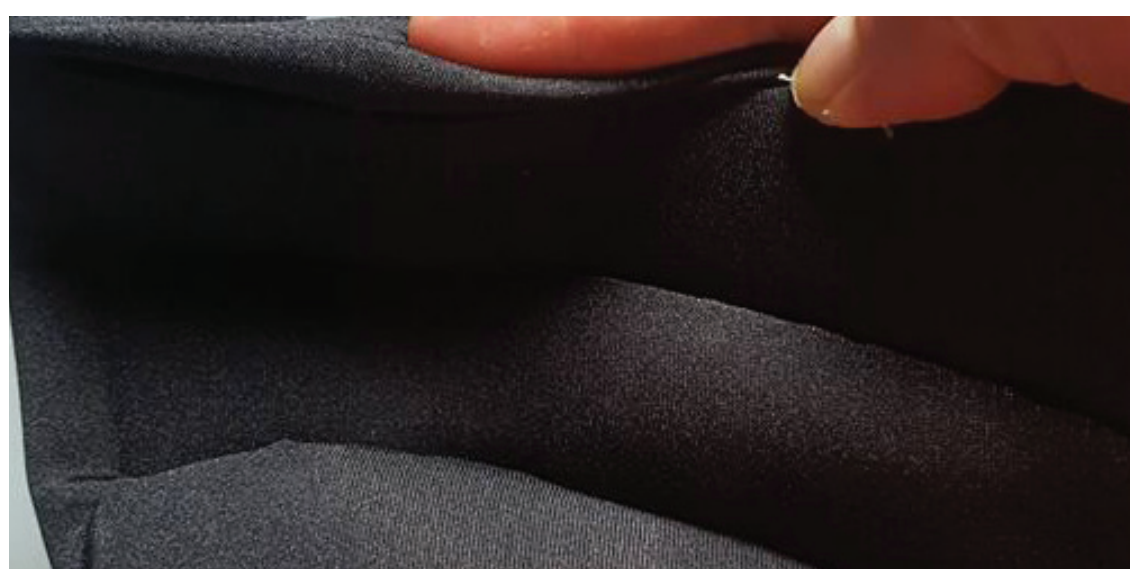




\section{Reflection}

The final 3d prints demonstrated compelling protective potential. Designed with an empty midsection made up of, (a) a flat base with $.5 \mathrm{~mm}$ height, used primarily for welding the $3 \mathrm{~d}$ print to the textiles effectively and (b) a top surface to provide a $3 \mathrm{~d}$ printed clean finish. Through these prints, findings demonstrated that prints with greater height were more impact responsive but became rigid, heavy and lost its ability to conform. When the height was reduces the models became responsive to impact but were more ergonomic. With further $3 \mathrm{~d}$ printing exploration with issue can be solved and refined to the design context. 
Resolving the Model

Chapter 7 
This phase includes the utilization of renders and photography to assess the aesthetic and function as well as the composition of the resolved models. Exploring various ways of how the finalised model can be composed onto the body in combination with the root/ vein protective system. Moreover, discussing the final design decisions and how they contribute to the design challenge. 


\section{Creating a Champion}

The thesis focusses on ways of enhancing sports performance through perception of form and colour. Perception considerations were introduced in the final design phase. Investigating how the final model can be altered slightly to stimulate enhanced sports performance. By increasing the protective skin thickness the protective quality is improved, and the physique of the athlete can be enhanced. This was explored because being perceived as more muscular can intimidate the opponent. Thus, lowering the self-confidence of the opponents while increasing the confidence of the user. Moreover, exploring how certain forms can create caution or distress, to negatively influence the opposition's performance.

To emphasise this, colour can be introduced. For example, applying red as it functions as a dominance cue, according to Hill and Barton (2005) competitors who wear red have a higher success rate compared to blue. As red is perceived as dominant, aggressive and intimidating. Furthermore, black is used through out the design development as study done by Mark G. Frank and Thomas Gilovich at Cornell University showed that teams who wear black uniforms were more inclined to engage in aggressive behaviour. The objective was to exploit this within contact sports, to enhance sports performance. Developing the 'To the roots' design approach in terms of colour selection. Using the red to symbolise veins and identity bringing forth motivation and confidence. On top of this, introducing gold with the red to encourage the user to honour his/her personal growth as well as realising they are champions. 


\section{Design Perception}

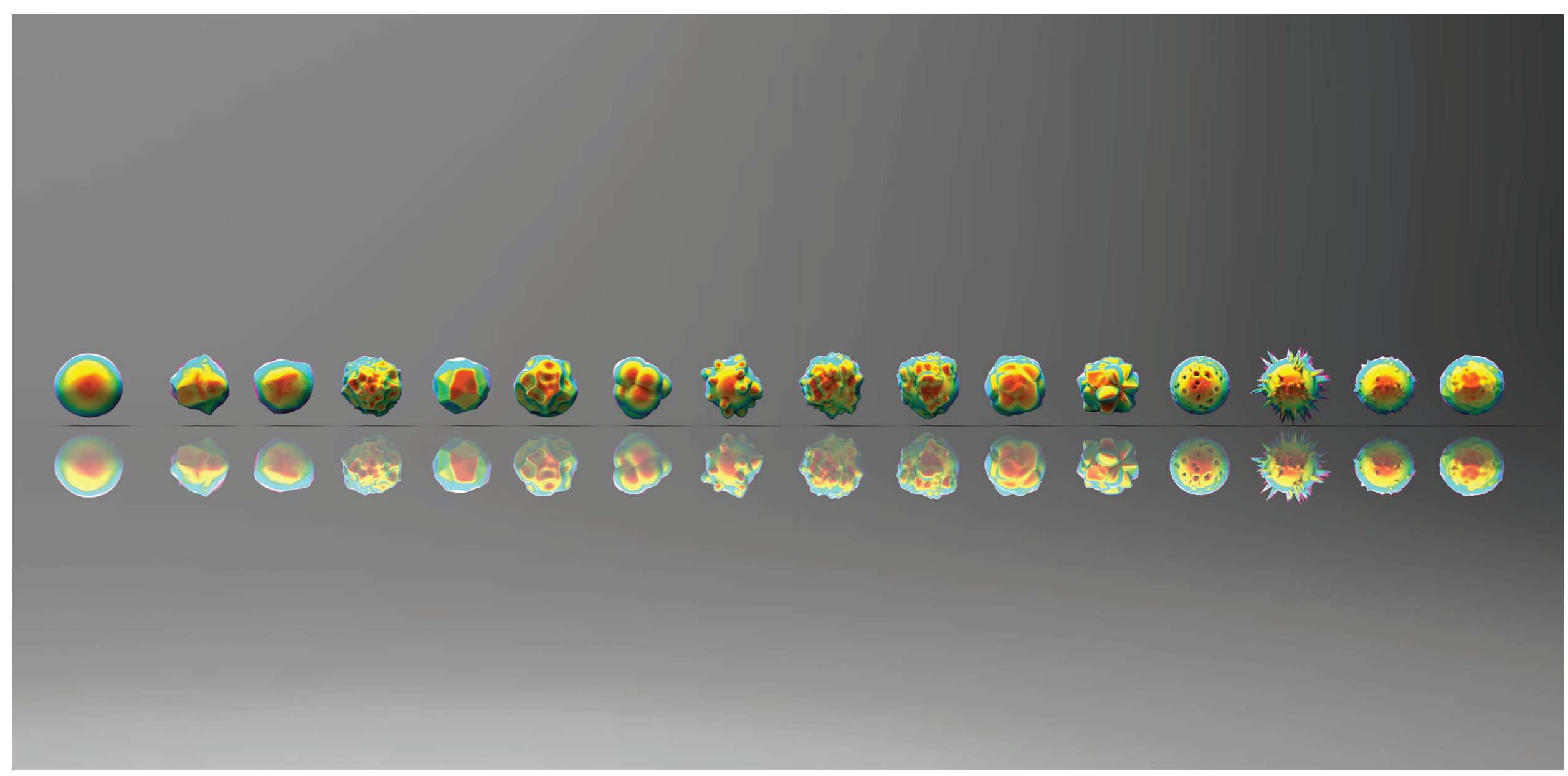

Figure 7.1

Exploring different surface qualities in order to explore perceptions. 

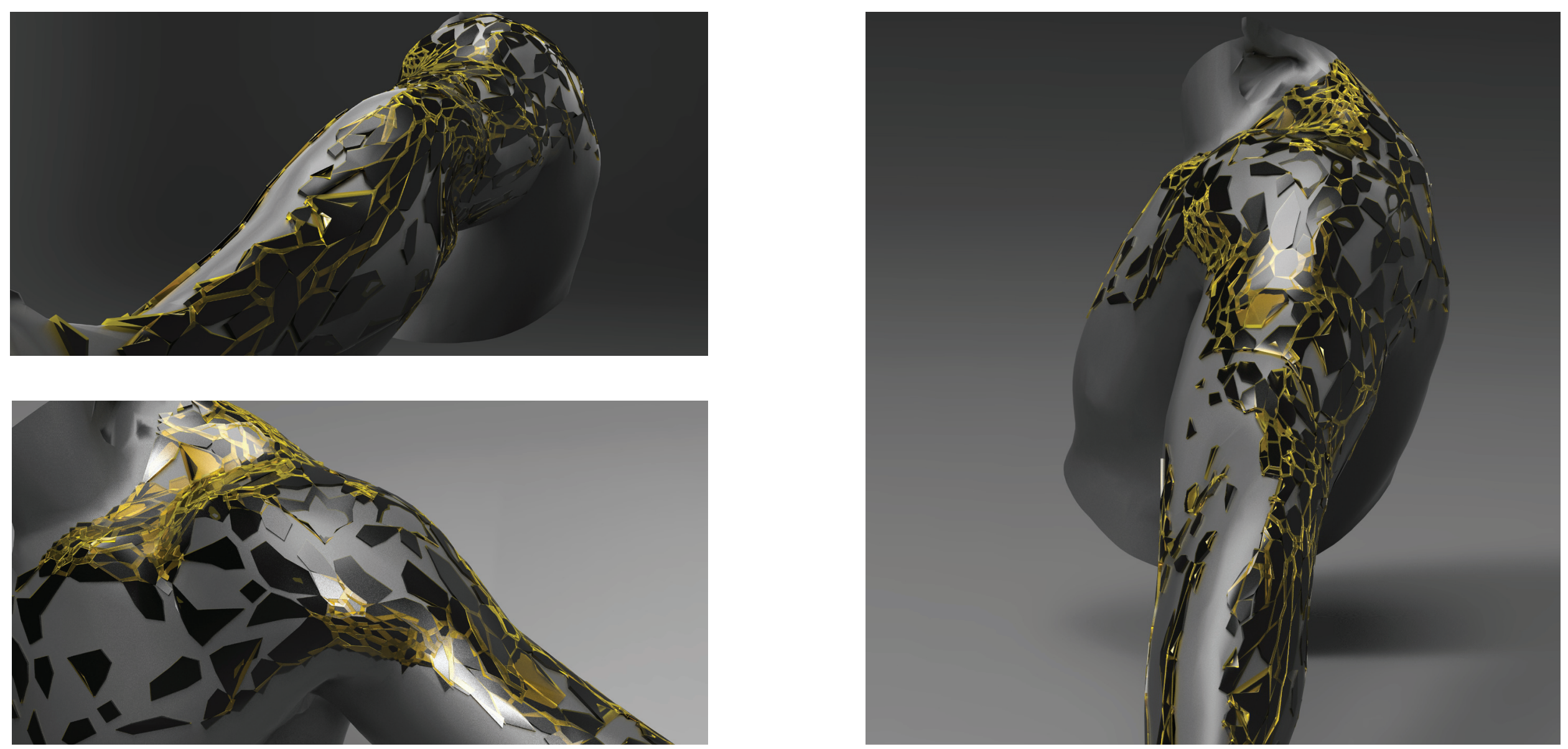

Figure 7.2

Altering the scale configuration on the body to produce a more organic outcome 

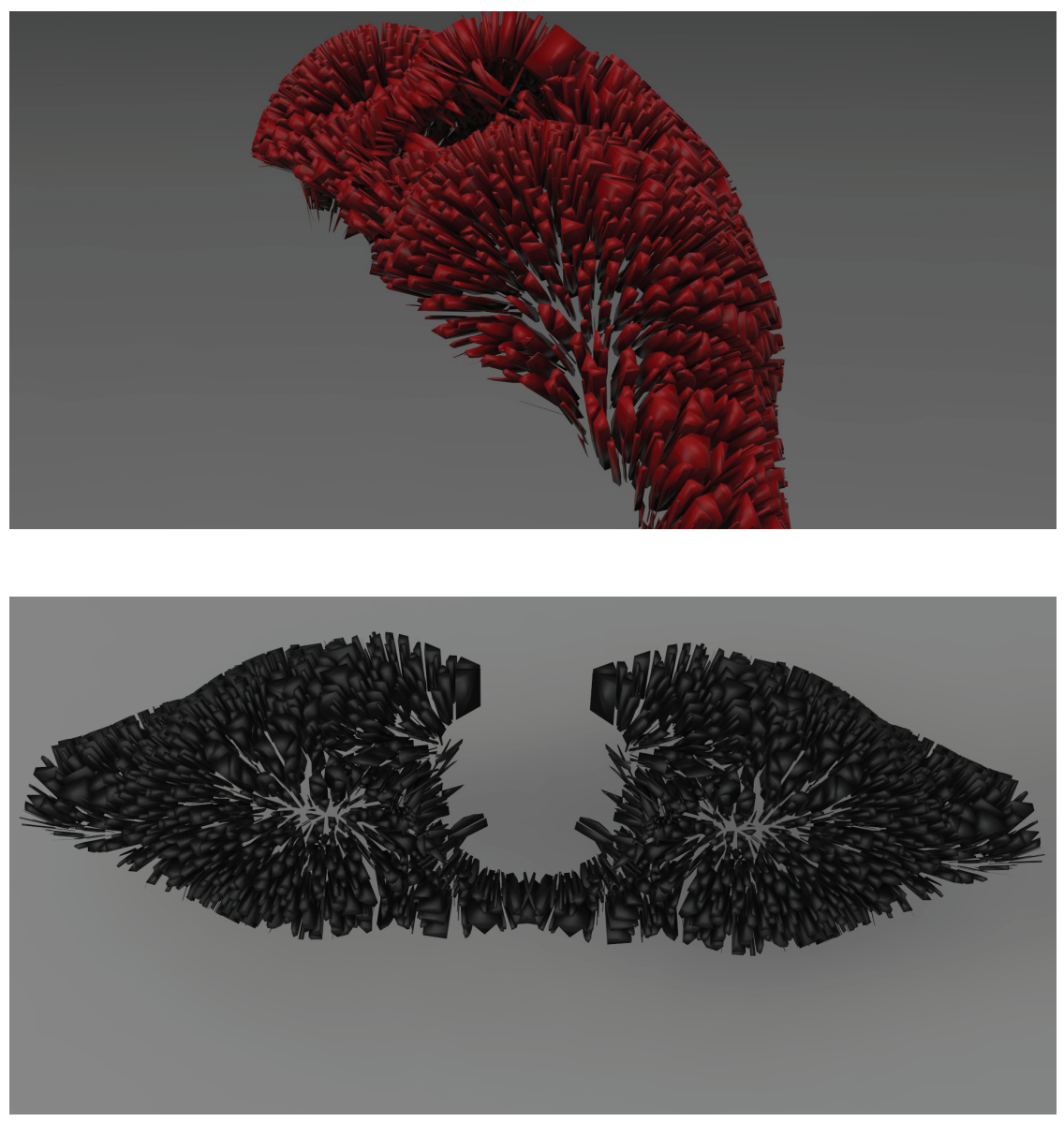

Figure 7.3

Exaggerating the scale extrusion to analyse of the protective skin can be perceived through aesthetic.

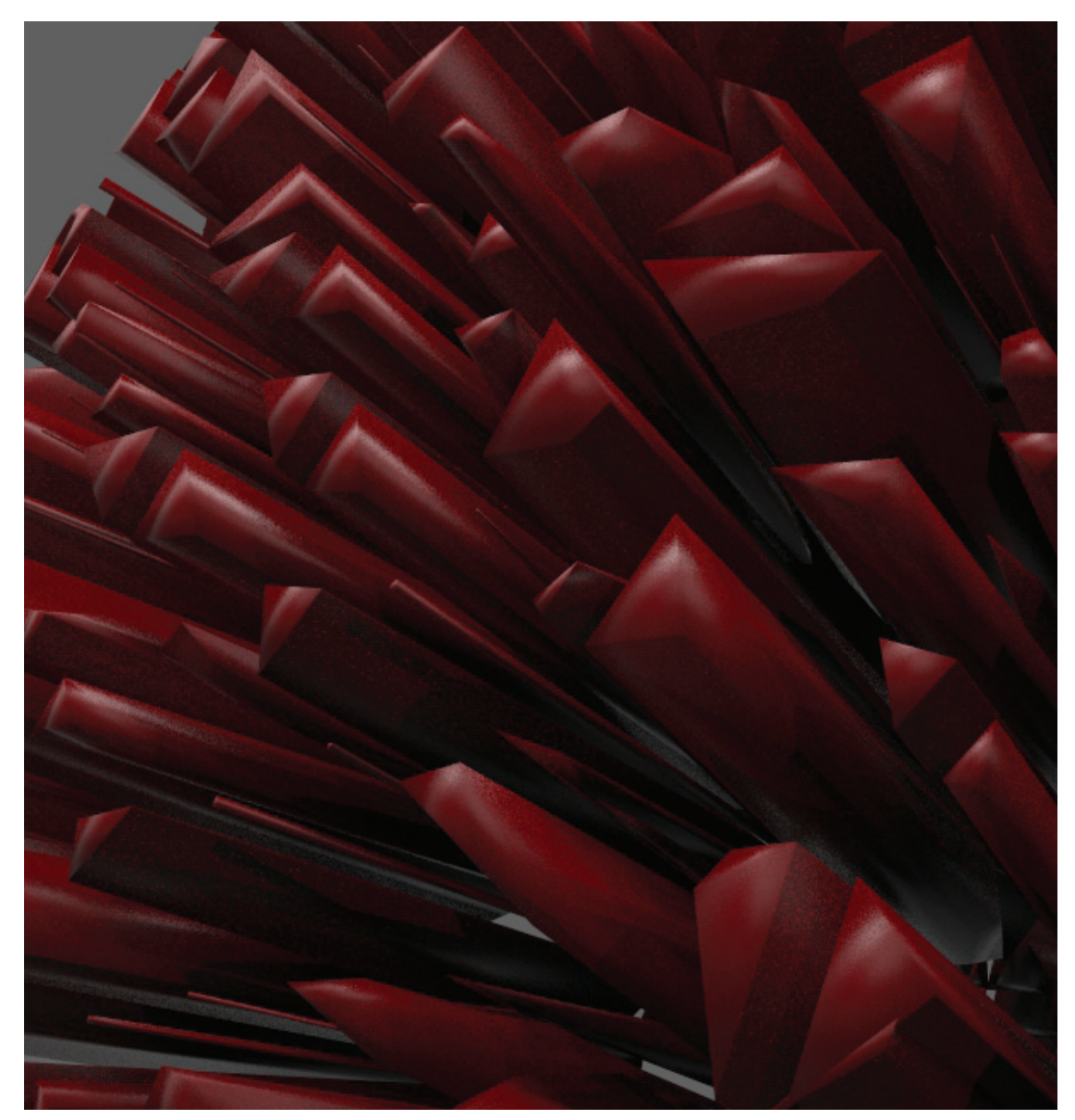

Figure 7.4

Introducing colour to the scales in order to determine a change in perception. 

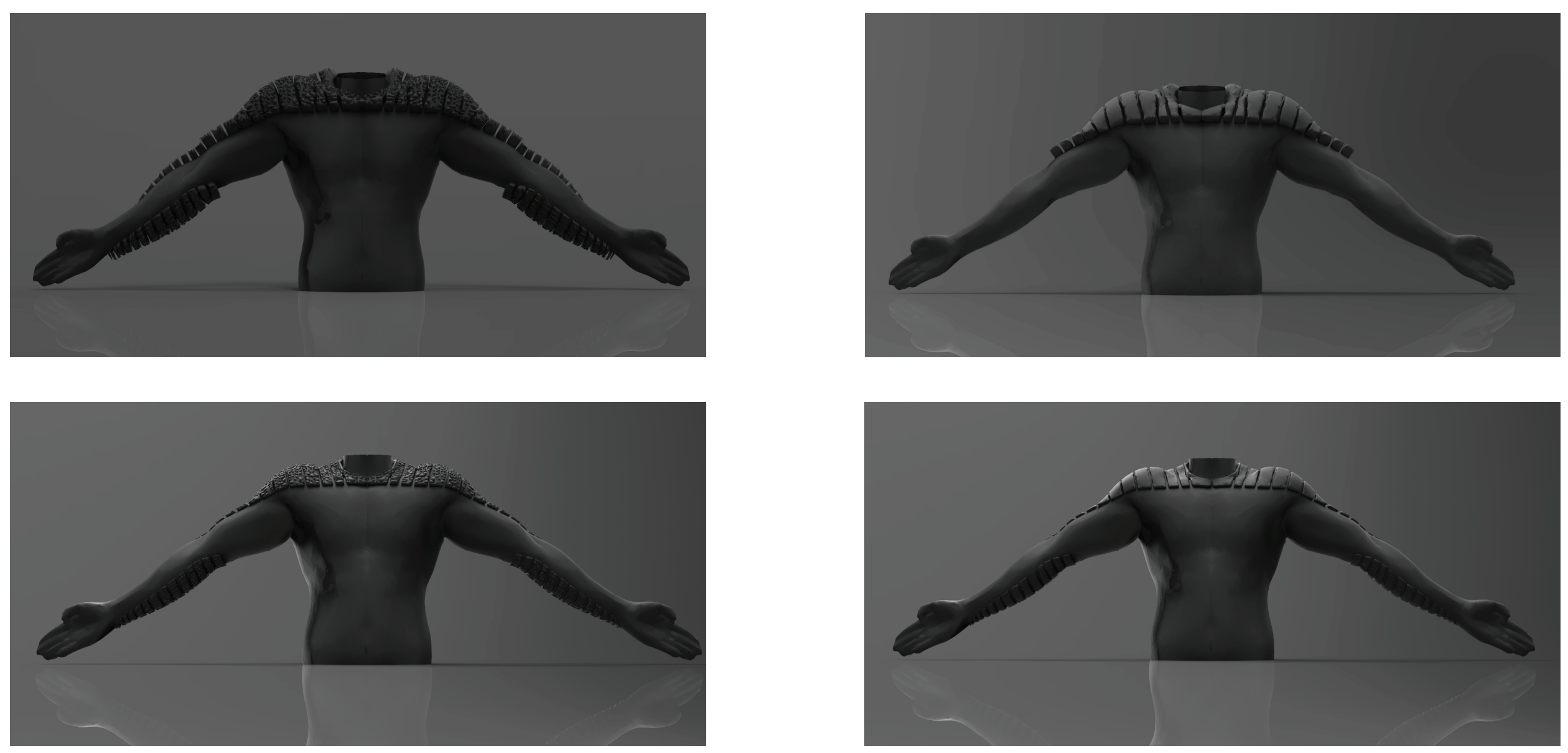

Figure 7.5

Creating variation in protective padding thickness to enhance the size of the shoulders. 

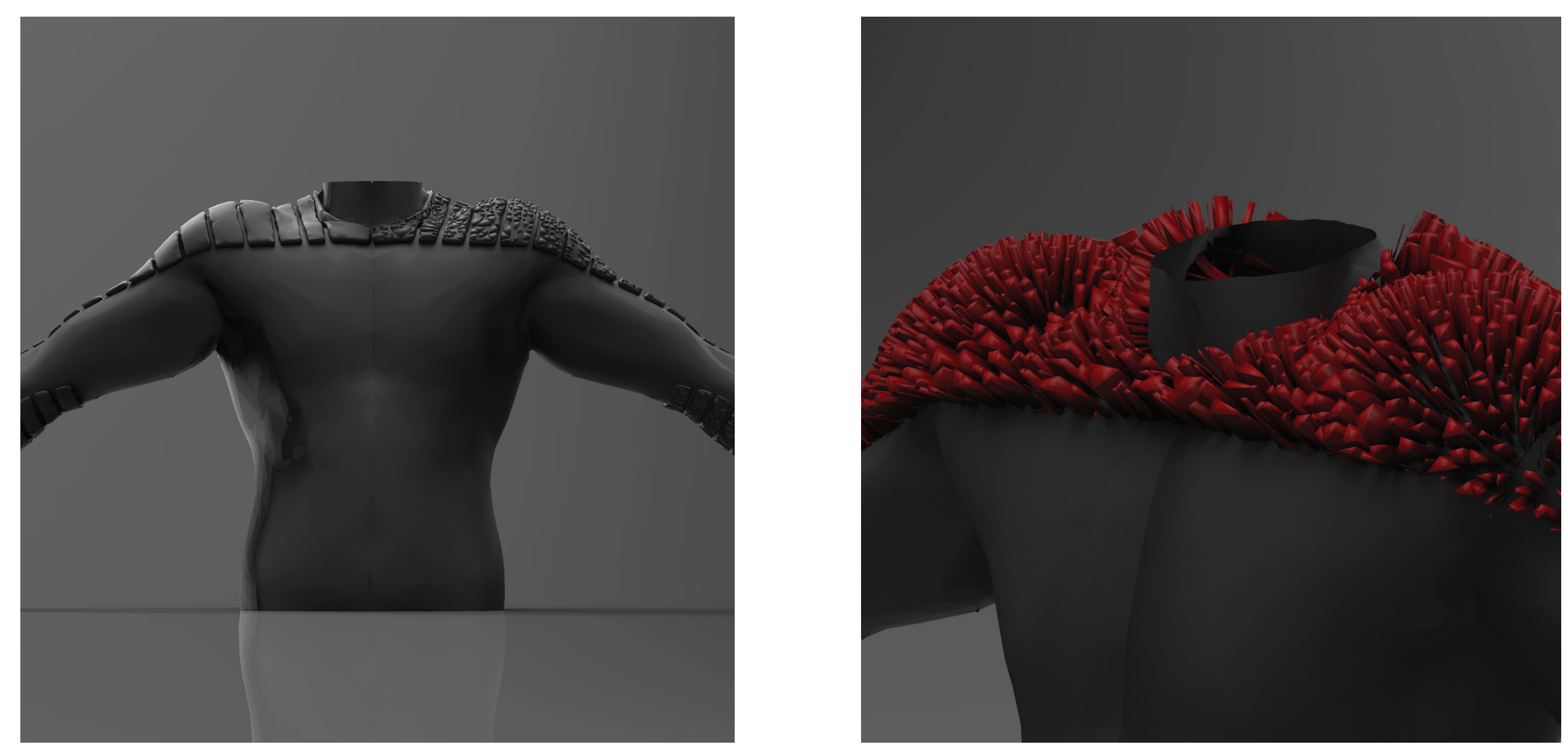

Figure 7.6

Comparing the smooth surface finish with the scale finish, to determine which composition is perceived as more protective, 


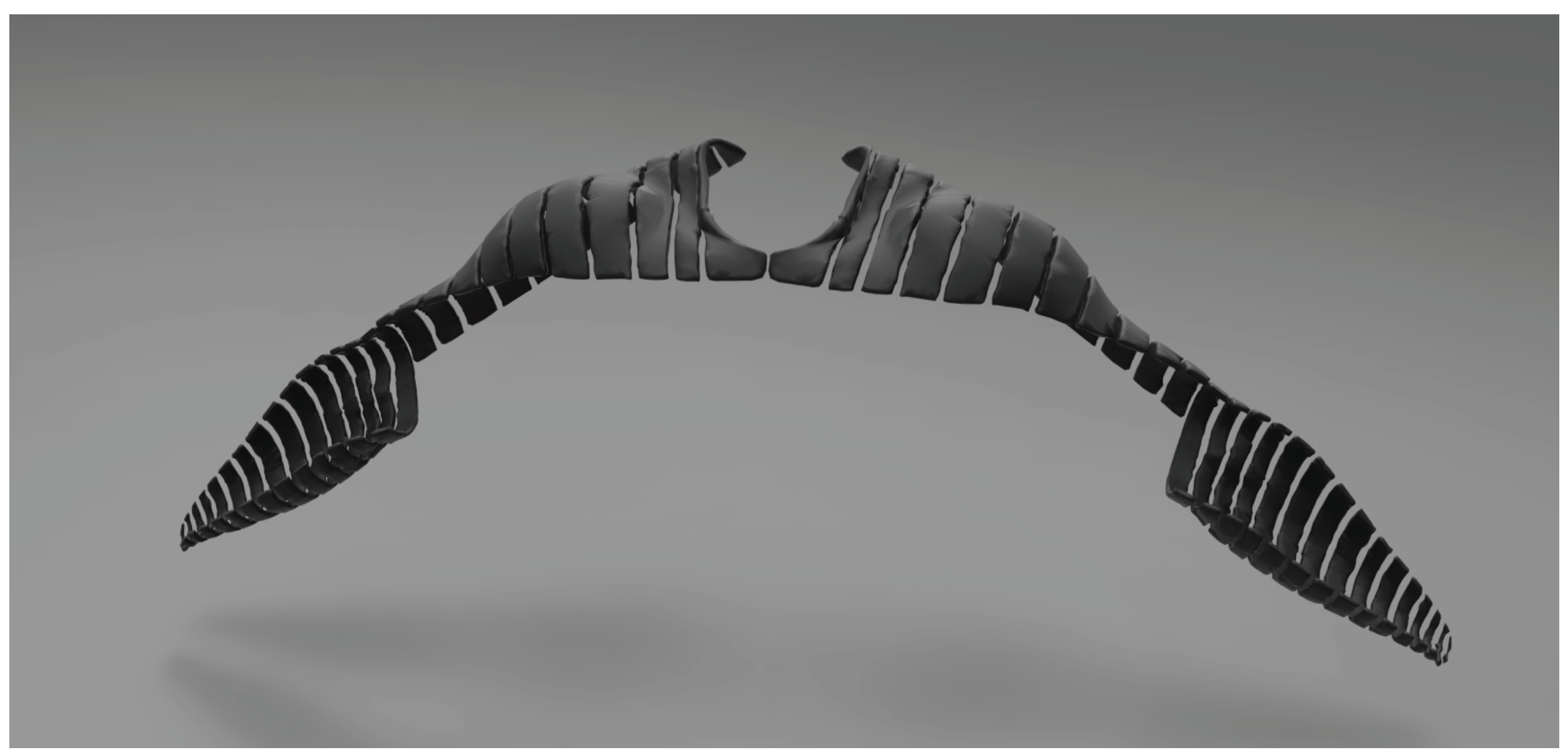

Figure 7.7

Final completed base design model. 

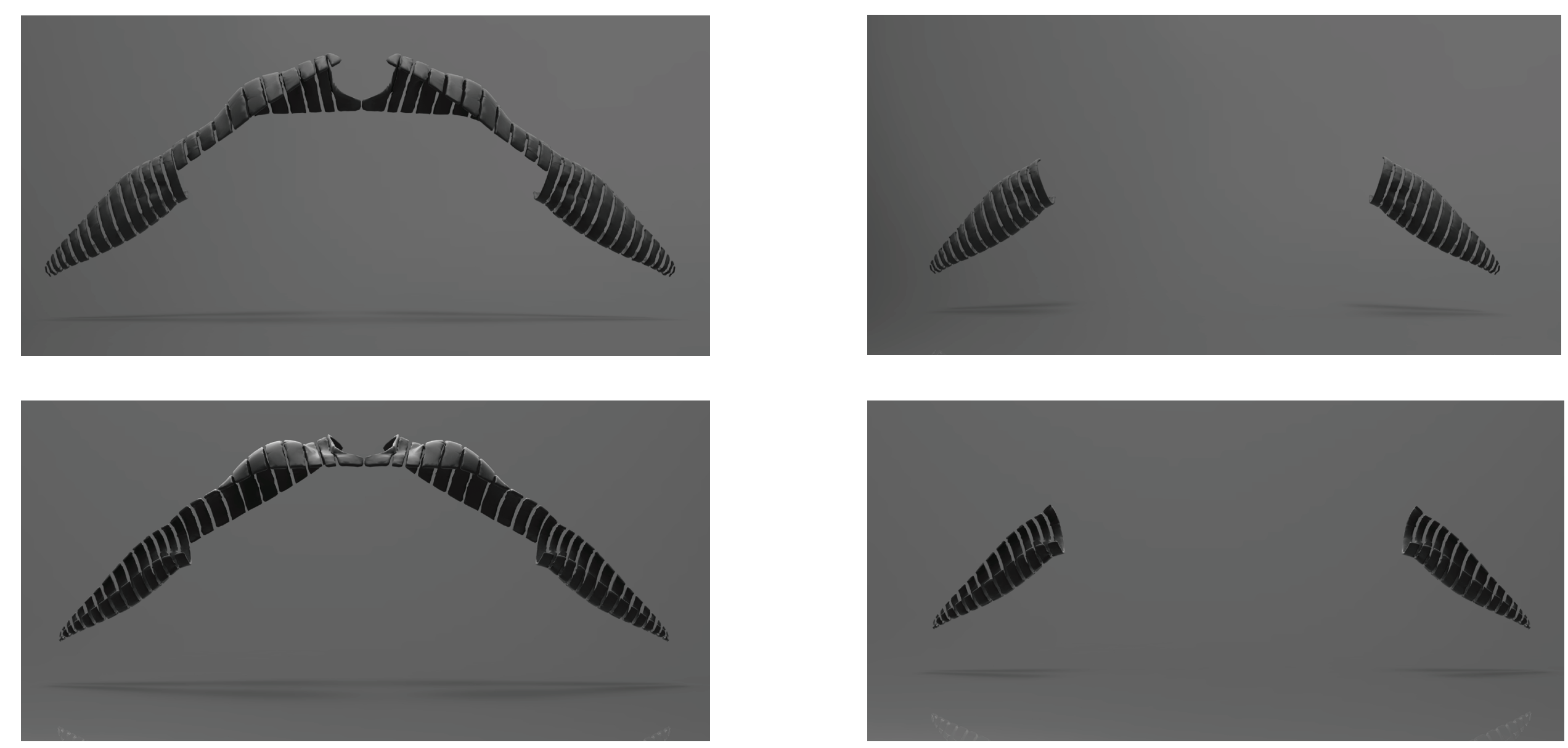

Figure 7.8

Final base model, communicating form the front and back view. 


\section{Final Design}

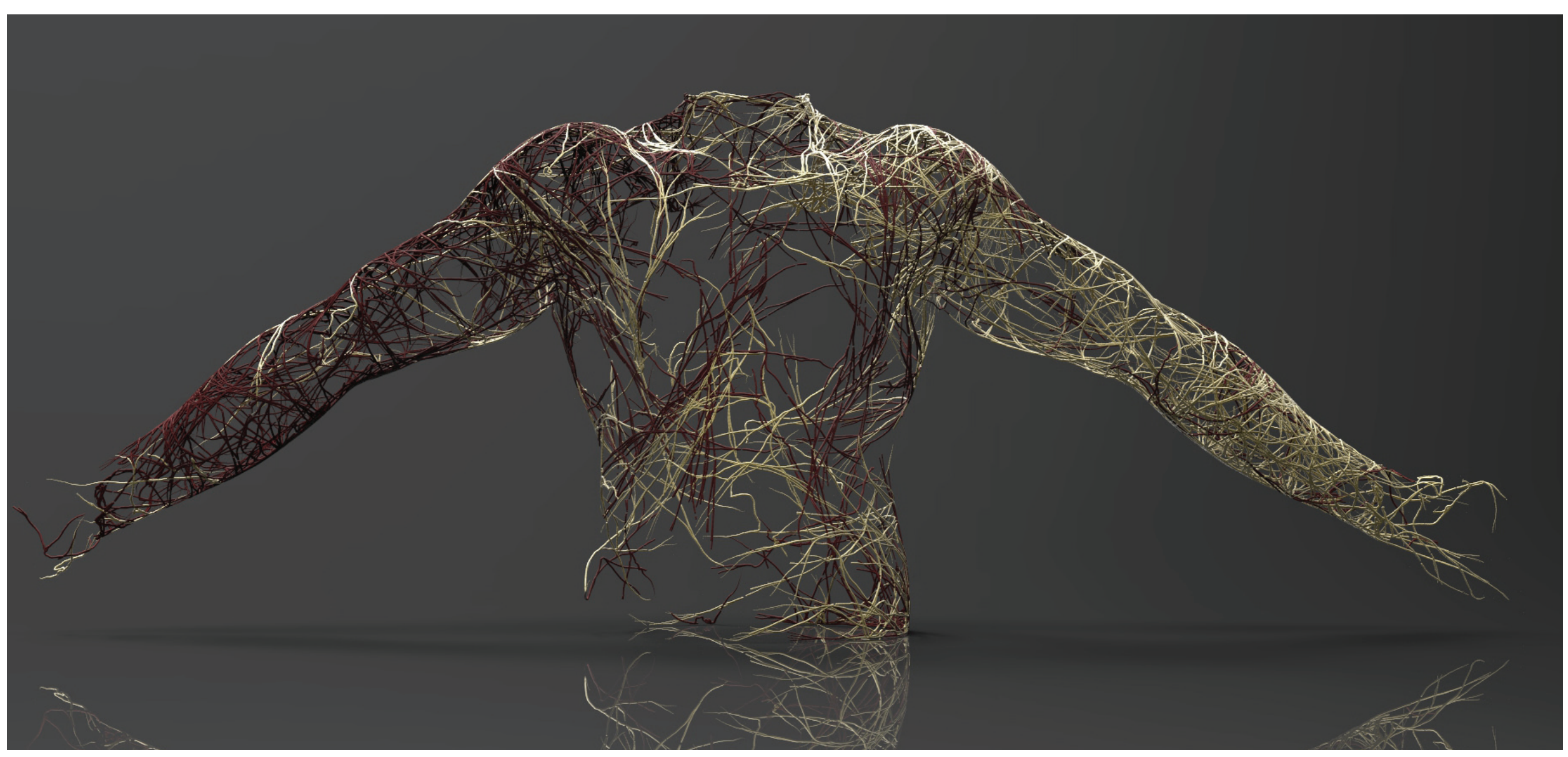

Figure 7.9

Resolved vein systems with colour incorporated, to respond to design perception. 


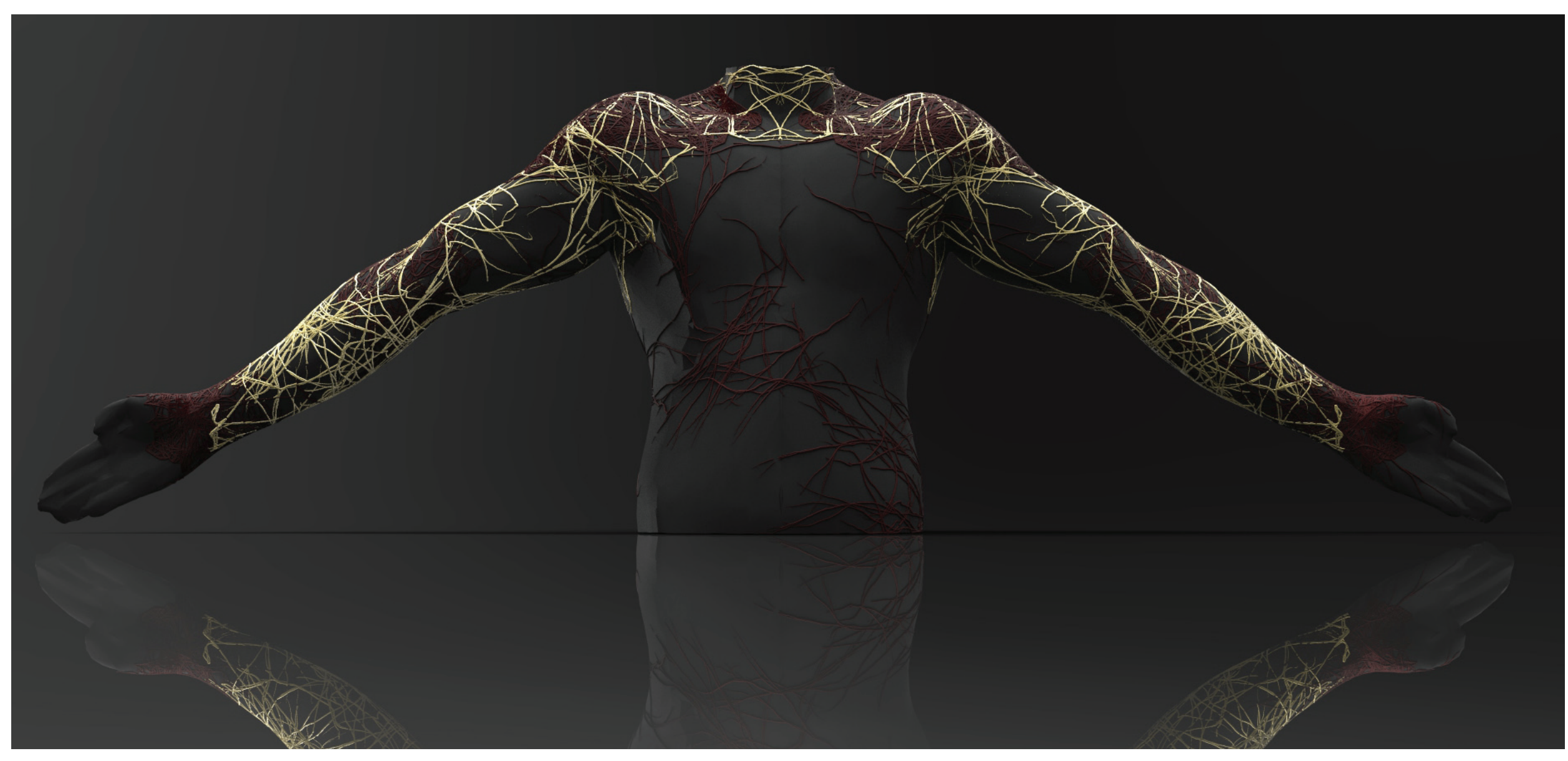

Figure 7.10

Front view of the resolved root/vein system. 


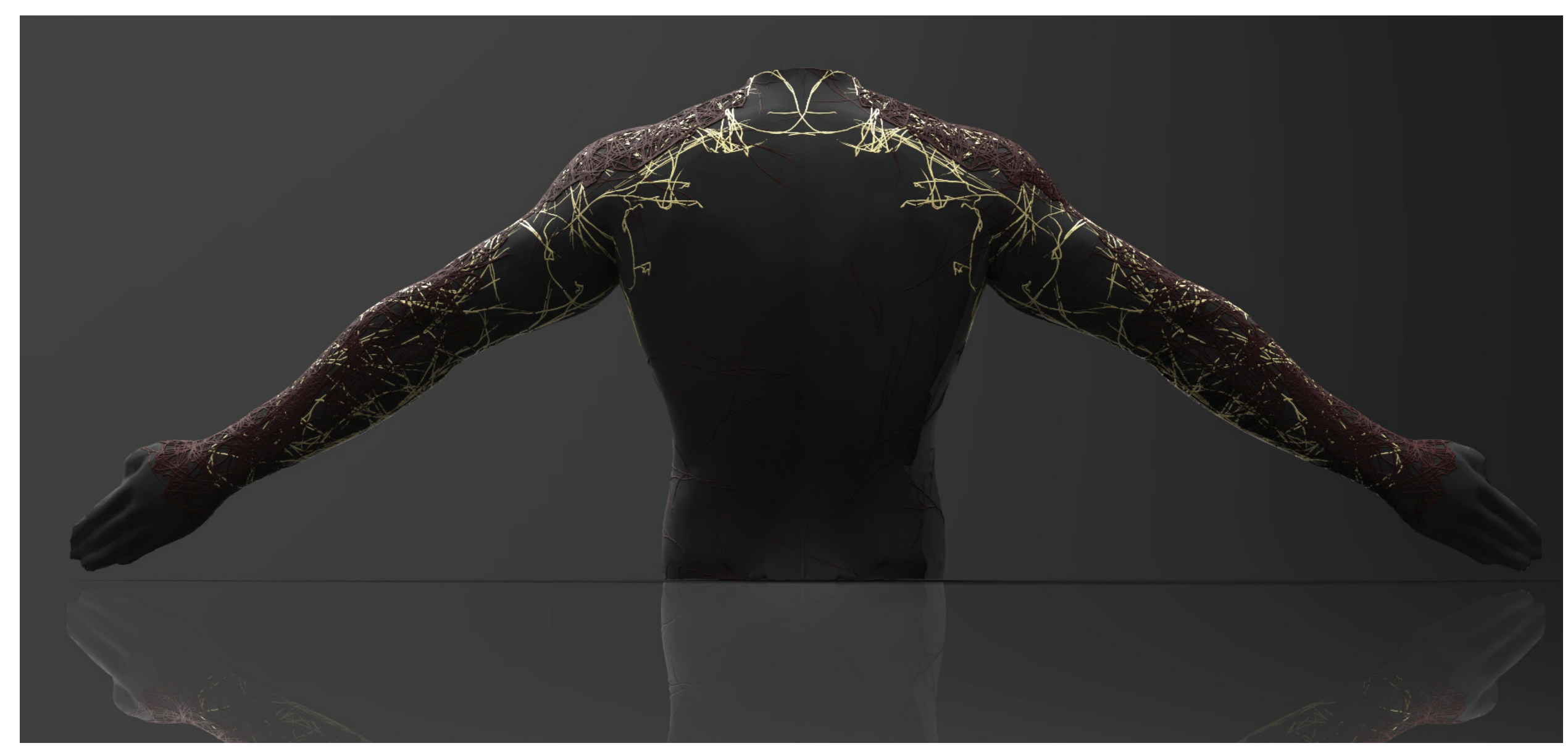

Figure 7.11

Rear view of the resolved root/vein system. 

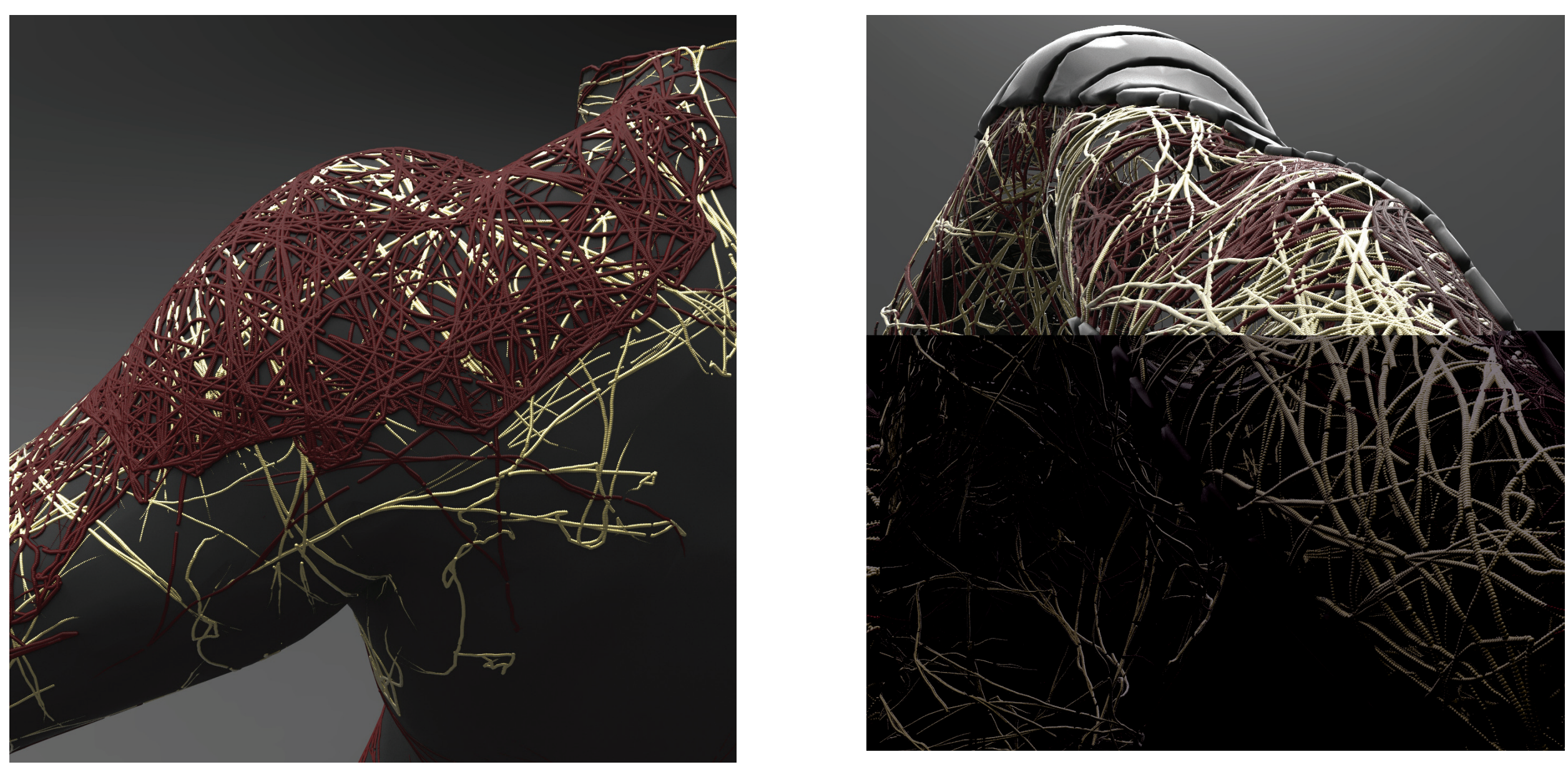

Figure 7.12

Close-ups showing the structural components of the final design compositions. 

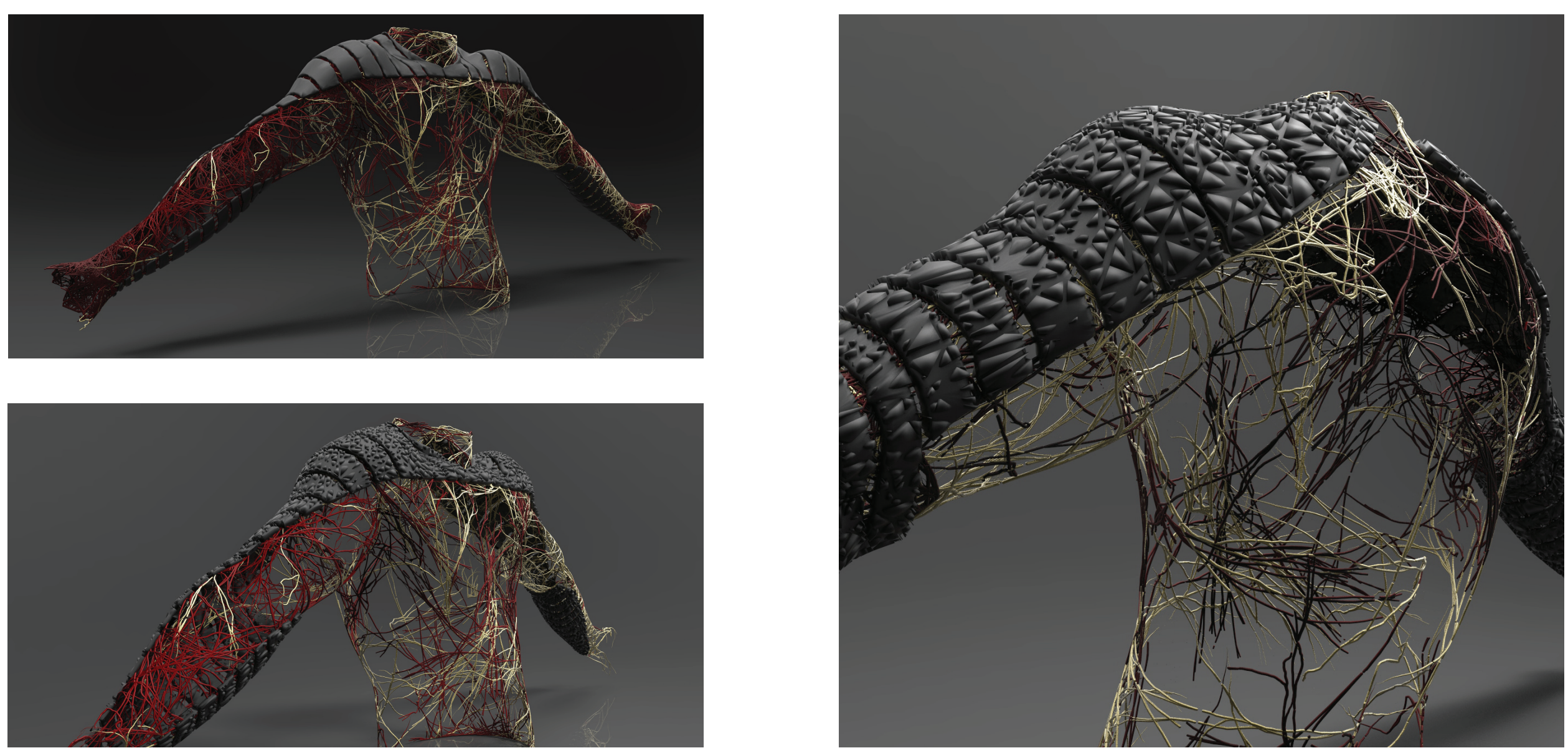

Figure 7.13

Renders of the completed model in respect to perception. 


\section{Physical Model}

In order to evaluate the functionality of the design, a physical model was created. This included $3 \mathrm{~d}$ printing the final form and applying it to a compression garment. Through this the garment was able to be worn and reflected upon. Analysing the final outcome in conjunction with the digital model and its design intentions. This process included, evaluating the $3 \mathrm{~d}$ printed skin during dynamic movements and selective contextual posing. Furthermore, this reflective process will determine how credible the design approach was. 

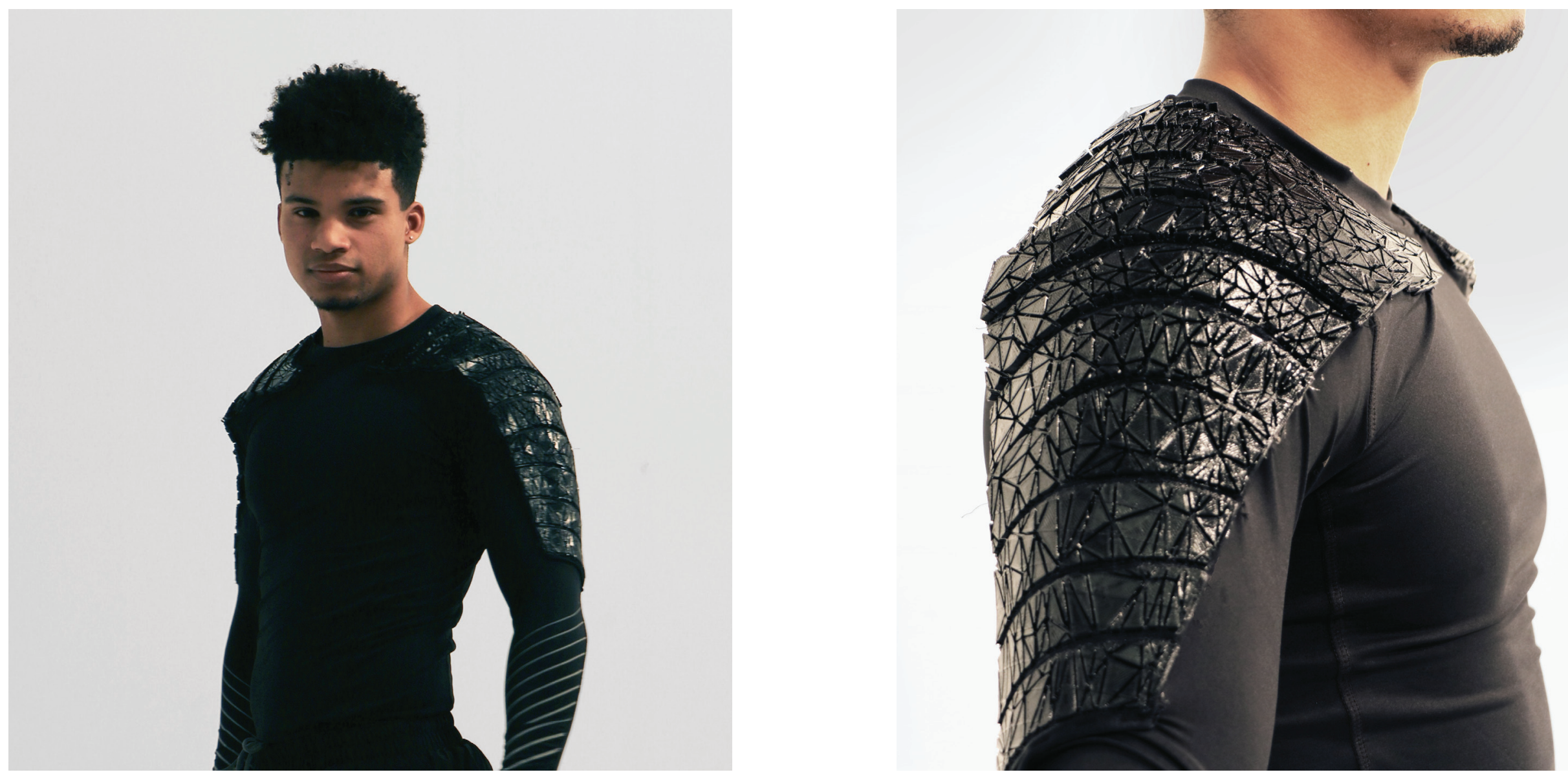

Figure 7.14

Photographs displaying how the physical model conforms to the body. 


$$
15
$$



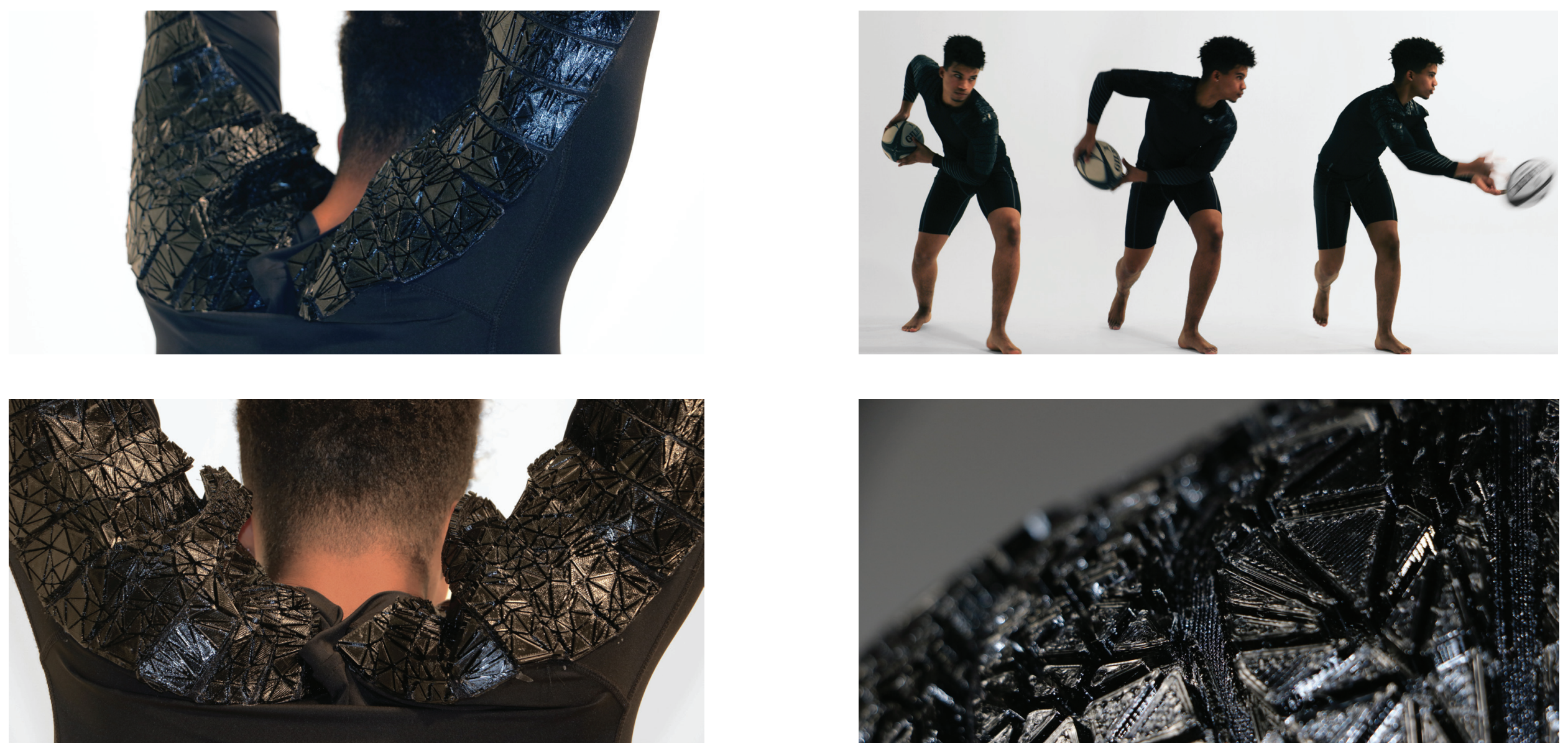

Figure 7.16

Investigating the dynamic qualities of the physical model through body moevements 


\section{Reflection}

The physical model showed effective contouring capability, moulding to the arms and shoulders effectively, suggesting a successful approach to customisation. When dynamic movement was introduced the skin responded well, with panels folding and digital scales flexing with the body. Therefore, concluding the digital scale approach to be effective for ergonomic purposes. In saying this the final model can be refined further to facilitate more efficient dynamic flow. Nonetheless, no apparent dynamic limitations were present due to the flexibility of the material, however the skin was noticeably heavier in comparison to traditional foam rugby sports padding. The final model displayed efficient durability which was emphasised by its rough exterior aesthetic. However, due to the simplicity of the design and customised capability it integrated well with the human form. The physical model demonstrated $3 \mathrm{~d}$ printing to be a compelling approach to the development of protective sportswear. 


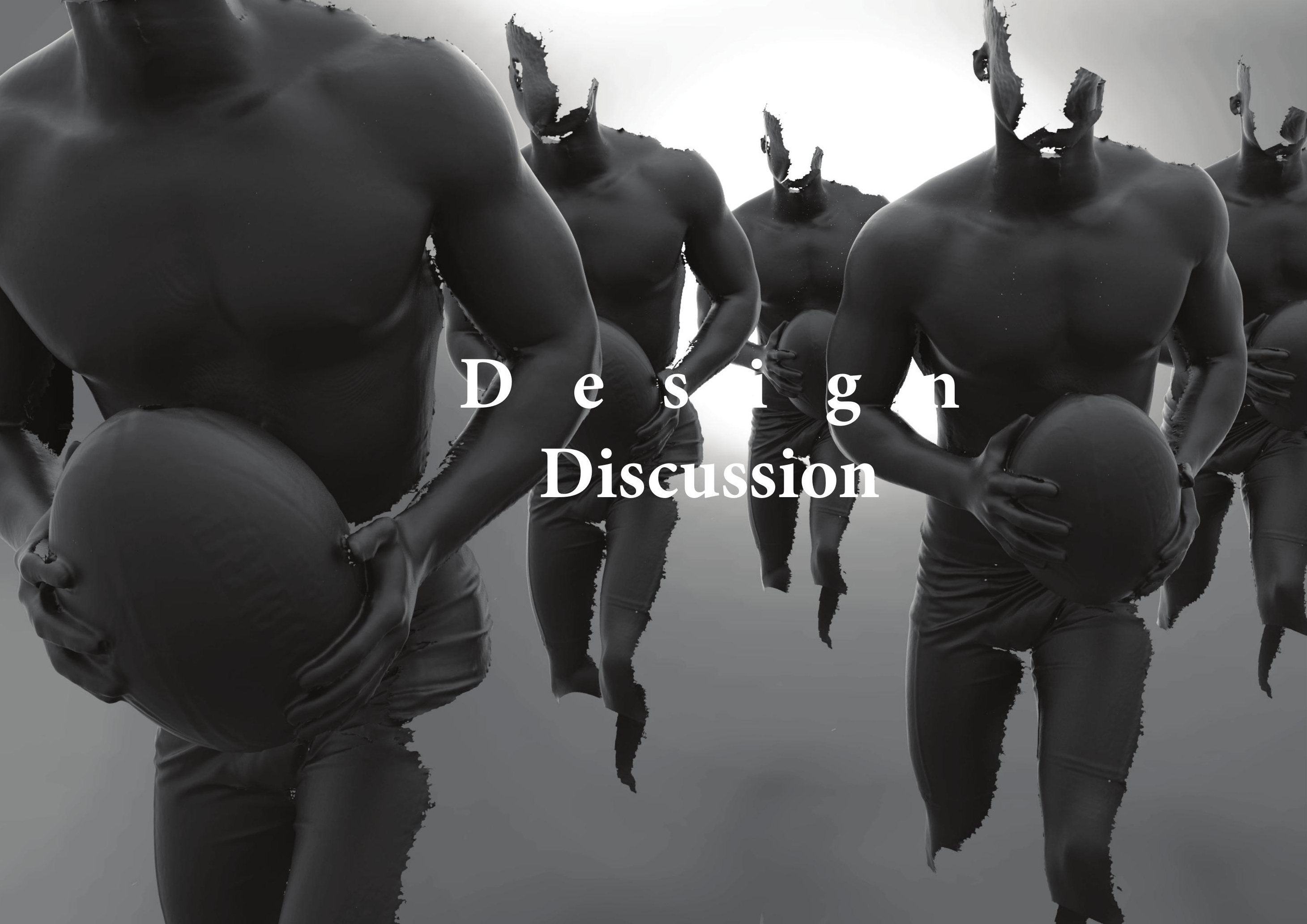



The design process had various discoveries, successes and failures. Aspects in this study which lacks completion or can be further researched and explored. These elements will be discussed in this chapter.

Research through design demonstrated the durability of the $3 \mathrm{~d}$ printed skin. The TPU $3 \mathrm{~d}$ printing filament proved to be robust, displaying efficient dynamic and flexibility in conjunction to the appropriate design decisions. The two textile and $3 \mathrm{~d}$ printing filament presented cohesive unification, as the appropriate tensile strength and flex of the $3 \mathrm{~d}$ printed model complimented each other through dynamic function. The $3 \mathrm{~d}$ printed design responded to the flexibility of the textiles and vice versa. Flexible $3 \mathrm{~d}$ printing filament was essential to comfort and impact functionality, correlating to traditional contact sports protective padding. Due to the complexity of the protective root/ vein design, it was proven too difficult to produce with the provided FDM printers. Therefore, this remained a digital model and was analysed speculatively based on secondary research, in terms of protective and perception qualities.

3D scanning the human figure was essential to creating the skin, this allowed the finished model to be customised to the geometry effectively and helping create and recreate models efficiently and effectively. Embedding the digital scales on top of the impact models created creases or folds needed to provide efficient dynamic freedom as well as restriction. Utilizing this strategy to create protective scales proved successful, on top of this growing an impact protective structure through FDM 3d printing displayed innovative possibilities if done correctly. Support 3d printing material played large role in creating viable protective structures that can be tested and evaluated further for production.

With the use of $3 \mathrm{~d}$ modelling in combination with $3 \mathrm{~d}$ printing, aspects such as perception can be further explored as well as how much impact is absorbed by the skin. Perception can be studied more in depth looking into how the form and colour of the model can stimulate a controlled response. Impact absorption can be researched and controlled further through analysing the programmed support structure software in regards to how the support structures are formed and why. Through this, creating a viable structure which will not support the model but can respond to impact. 
Throughout the design process, the bond between the $3 \mathrm{~d}$ printing filament and textile were effective. However, as these prints contain little extruded scales, how well these will respond to continuous heavy sports contact may bring forth design errors. As this thesis focused most on how a meaningful design can be created through $3 \mathrm{~d}$ printing, disregarding the quality of the design within its given context, problems may arise when tested. Issues such as the adhesive quality between the two materials, perception of the skin, validity in terms of impact absorption, effectiveness of the structural vein system, dynamic and ergonomic quality within the context. However, this thesis applied a speculative design approach in order to discover a protective and performance enhancing strategy based on contemporary technology. Therefore, no testing besides research through design were practiced as the aim of this process was to investigate how a $3 \mathrm{~d}$ printed skin can be produced.

With some improvements this approach to a protective and performance enhancing skin can become a design successful enough to be manufactured in the future. The experiments demonstrated protective opportunities and $3 \mathrm{~d}$ printing to be a credible approach to creating a skin that protects athletes during contact. Findings showed features plausible enough for further development and testing. These features and or design successes can be exploited to create a skin not only for application in sports but also in fields such as the military. With the range of $3 \mathrm{~d}$ printing filaments and textiles, the thesis findings can be further explored and elaborated upon 


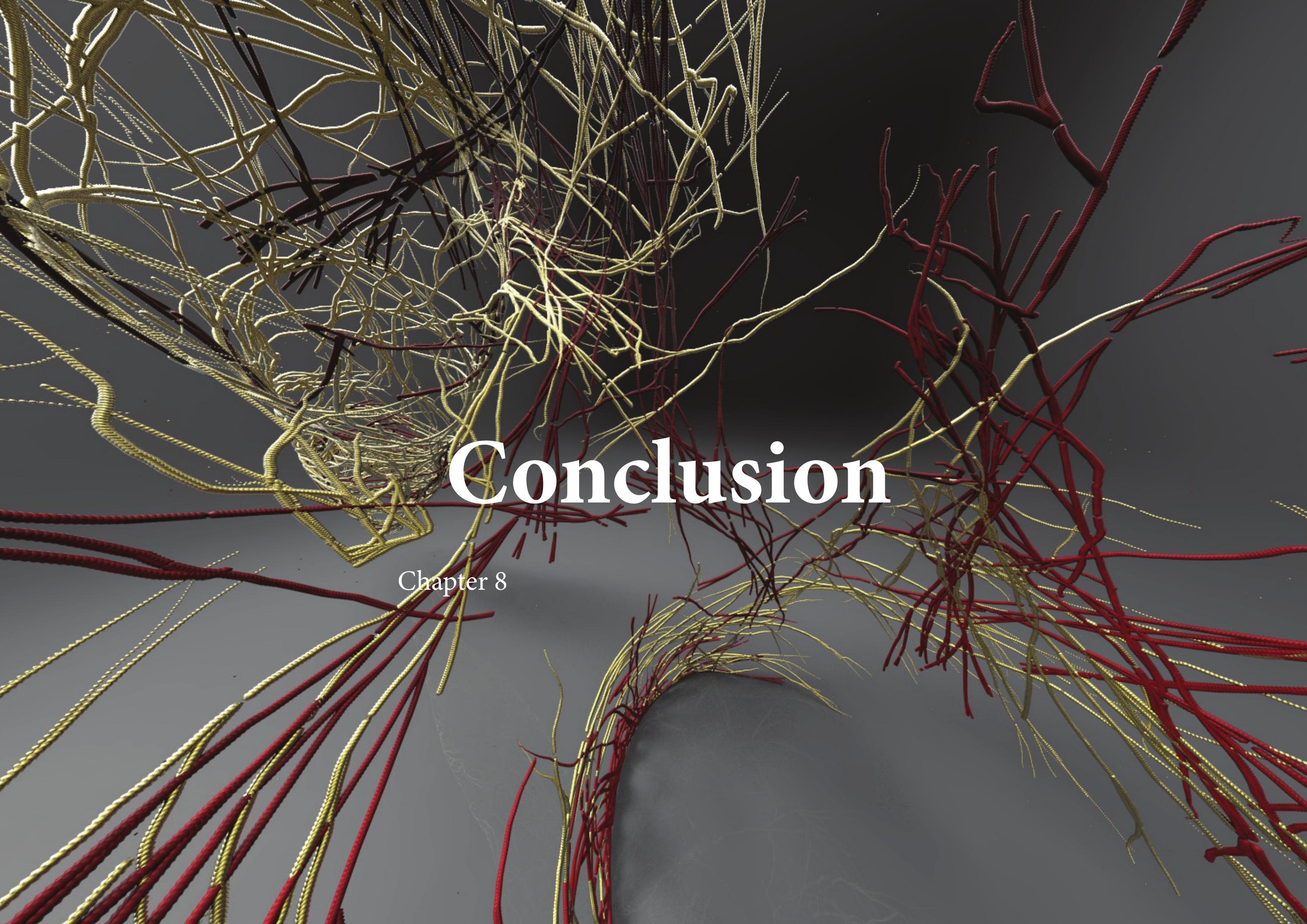


Often athletes at the professional level believe in ritual and superstition and these behaviours can affect their motivation to perform. The mentality is grown from their safety and security of their body and well-being.

This research was based on my interest in human activities and how these activities can be communicated through visual qualities of how people look and feel. I was inspired by companies such as Adidas and Nike and wanted to produce this same inspiration through my work. The notion of $3 \mathrm{~d}$ printed FDM skin has been unexplored as companies concentrated on high print methods. In the completion of this research, questions were raised on how this work would inform sportswear design and where it could lead to in the future. Through the use of speculative and growth methodology, the research serves to show how innovation can occur and inspire further research into the enhancement of an athletes sports performance and transform them into a champion. 


\section{Bibliography}

Azeemi, S. T. Y., \& Raza, S. M. (2005). A Critical Analysis of Chromotherapy and Its Scientific Evolution. Evidence-Based Complementary and Alternative Medicine, 2(4), 481-488. https://doi.org/10.1093/ecam/neh137

Baker Miller Pink Hoodie: Engineered for relaxation and recovery. (n.d.). Retrieved October 19, 2017, from https://www.vollebak.com/product/ baker-miller-pink-hoodie/

Born, D.-P., Sperlich, B., \& Holmberg, H.-C. (2013). Bringing Light into the Dark: Effects of Compression Clothing on Performance and Recovery. International Journal of Sports Physiology and Performance, 8(1), 4-18. https://doi.org/10.1123/ijspp.8.1.4

Coyle, S., Morris, D., Lau, K. T., Diamond, D., \& Moyna, N. (2009). Textile-Based Wearable Sensors for Assisting Sports Performance. In 2009 Sixth International Workshop on Wearable and Implantable Body Sensor Networks (pp. 307-311). https://doi.org/10.1109/BSN.2009.57 D3O - We protect people and their things. (2016, August 25). Retrieved October 10, 2017, from https://www.d3o.com/

Dehydration and its effects on performance. (n.d.). Retrieved July 16, 2018, from http://www.humankinetics.com/excerpts/excerpts/dehydration-and-its-effects-on-performance

Does sweat-fighting athletic gear work? (n.d.). Retrieved July 16, 2018, from http://www.slate.com/articles/sports/sports_nut/2005/09/no_ sweat.html

EXOS | Human Performance | Services, Spaces \& Technology. (n.d.). Retrieved October 10, 2017, from http://www.teamexos.com/ 
Edelson. D (2002). Design Research : What we learn when we engage in design. Journal of the learning sciences. 11(1), 105-121. Retrieved from https://www.cs.uic.edu/ i523/edelson.pdf

Farahangiz, S., Mohebpour, F., \& Salehi, A. (2016). Assessment of Mental Health among Iranian Medical Students: A Cross-Sectional Study. International Journal of Health Sciences, 10(1), 49-55.

FrankGilovich1988.pdf. (n.d.). Retrieved from http://www2.psych.ubc.ca/ schaller/Psyc591Readings/FrankGilovich1988.pdf

Hydrodynamic function of biomimetic shark skin: Effect of denticle pattern and spacing | Request PDF. (n.d.). Retrieved July 16, 2018, from https:// www.researchgate.net/publication/284171584_Hydrodynamic_function_of_biomimetic_shark_skin_Effect_of_denticle_pattern_and_spacing Ingram, J., Dawson, B., Goodman, C., Wallman, K., \& Beilby, J. (2009). Effect of water immersion methods on post-exercise recovery from simulated team sport exercise. Journal of Science and Medicine in Sport, 12(3), 417-421. https://doi.org/10.1016/j.jsams.2007.12.011

j.1467-8721.2007.00514.pdf. (n.d.). Retrieved from http://journals.sagepub.com/doi/pdf/10.1111/j.1467-8721.2007.00514.x

Li, R. T., Kling, S. R., Salata, M. J., Cupp, S. A., Sheehan, J., \& Voos, J. E. (2016). Wearable Performance Devices in Sports Medicine. Sports Health, 8(1), 74-78. https://doi.org/10.1177/1941738115616917

lyj6.pdf. (n.d.). Retrieved from http://cg.cs.tsinghua.edu.cn/papers/lyj6.pdf

LZR Racer. (2017). In Wikipedia. Retrieved from https://en.wikipedia.org/w/index.php?title=LZR_Racer\&oldid=805857716

MacRae, B. A., Cotter, J. D., \& Laing, R. M. (2011). Compression Garments and Exercise. Sports Medicine, 41(10), 815-843. https://doi. org/10.2165/11591420-000000000-00000

Moemeni Piri, S., Hashemi Afosi2, M., Rezaeinasab, A., Noroozi, S., Maghboli, S., \& Babai Mazreno, A. (2015). The Effects of Physical Educa- 
tion Course on Mental Health of Students in Bu-Ali Sina University, Hamedan-Iran. International Journal of Pediatrics, 3(2.1), 67-73. https://doi. org/10.22038/ijp.2015.4052

Mountain Bike Armor | VPD Protection | POC Sports. (n.d.). Retrieved October 19, 2017, from http://www.pocsports.com/eu/armor/mountain-bikearmor/

Ou, L.-C., Luo, M. R., Woodcock, A., \& Wright, A. (2004). A study of colour emotion and colour preference. Part I: Colour emotions for single colours. Color Research \& Application, 29(3), 232-240. https://doi.org/10.1002/col.20010

pdf.pdf. (n.d.). Retrieved from http://iopscience.iop.org/article/10.1088/1757-899X/87/1/012005/pdf

Rafts, Skirts and Brims! (n.d.). Retrieved July 16, 2018, from https://www.simplify3d.com/support/articles/rafts-skirts-and-brims/

Review: POC VPD Air Knee Pads - Winner Most Protection. (n.d.). Retrieved October 19, 2017, from http://singletrackworld.com/2017/07/reviewpoc-vpd-air-knee-pads-winner-most-protection/

Roberts, L. A., Raastad, T., Markworth, J. F., Figueiredo, V. C., Egner, I. M., Shield, A., .. Peake, J. M. (2015). Post-exercise cold water immersion attenuates acute anabolic signalling and long-term adaptations in muscle to strength training. The Journal of Physiology, 593(Pt 18), 4285-4301.

https://doi.org/10.1113/JP270570

sportsmedicine-3-1060.pdf. (n.d.). Retrieved from https://www.jscimedcentral.com/SportsMedicine/sportsmedicine-3-1060.pdf

STATSports | STATSports Viper | GPS Player Tracking and Performance Analysis. (n.d.). Retrieved October 18, 2017, from http://statsports.com/ viper/

Supports in 3D Printing: A technology overview. (n.d.). Retrieved July 16, 2018, from https://www.3dhubs.com/knowledge-base/supports-3d-print- 
ing-technology-overview

The Future of Biosensing Wearables | Rock Health | We're powering the future of healthcare. Rock Health is a seed and early-stage venture fund that supports startups building the next generation of technologies transforming healthcare. (n.d.). Retrieved July 16, 2018, from https://rockhealth.com/ reports/the-future-of-biosensing-wearables/

Tovey, M., Porter, S., \& Newman, R. (2003). Sketching, concept development and automotive design. Design Studies, 24(2), 135-153. https://doi. org/10.1016/S0142-694X(02)00035-2

Understanding and enhancing sports performance. (n.d.). Retrieved October 15, 2017, from http://www.usc.edu.au/research-and-innovation/medicaland-health-science/sport-and-exercise-science/understanding-and-enhancing-sports-performance

VICIS Pro. (n.d.). VICIS ZERO1 UNVEIL. Retrieved from https://www.youtube.com/watch?v=4bMOMf3S_EA

What is D3O. (2016, August 25). Retrieved October 10, 2017, from https://www.d3o.com/what-is-d3o/

What is: VPD? | POC Wall. (n.d.). Retrieved October 19, 2017, from http://news.pocsports.com/2013/05/03/what-is-vpd/

Xenith. (n.d.). EPIC+ Helmets. Retrieved July 15, 2018, from https://www.xenith.com/pages/epic-plus

Zainuddin, Z., Newton, M., Sacco, P., \& Nosaka, K. (2005). Effects of Massage on Delayed-Onset Muscle Soreness, Swelling, and Recovery of Muscle Function. Journal of Athletic Training, 40(3), 174-180.

ZERO1 2018 Reservation. (n.d.). Retrieved October 15, 2017, from https://shop.vicis.co/products/zero1 


\section{Figures and Tables}

All images photographed or created by author, excluding:

Figures $7.14-7.16$

Images provided by Fenella Richards 